\title{
FUSION SYSTEMS
}

\author{
MICHAEL ASCHBACHER AND BOB OLIVER
}

Abstract. This is a survey article on the theory of fusion systems, a relatively new area of mathematics with connections to local finite group theory, algebraic topology, and modular representation theory. We first describe the general theory and then look separately at these connections.

\section{Contents}

Introduction

1. Basic properties of fusion systems

2. A local theory of fusion systems

3. Linking systems, transporter systems, and localities

4. Interactions with homotopy theory

5. Interactions with representation theory

6. Generalizations

7. Open questions and problems

About the authors

References

\section{INTRODUCTION}

Let $G$ be a finite group, $p$ a prime, and $S$ a Sylow $p$-subgroup of $G$. Subsets $X$ and $Y$ of $S$ are said to be fused in $G$ if they are conjugate in $G$; that is, there exists $g \in G$ such that ${ }^{g} X:=g X g^{-1}=Y$. The use of the word "fusion" seems to be due to Richard Brauer, but the notion is much older, going back at least to Burnside in the late nineteenth century.

The study of fusion in finite groups played an important role in the classification of the finite simple groups. Indeed fusion even leaked into the media in the movie It's My Turn, where Jill Clayburgh plays a finite group theorist on the trail of a new sporadic group, talking to her graduate student (played by Daniel Stern) about the 2-fusion in her sought-after group.

Received by the editors December 27, 2015.

2010 Mathematics Subject Classification. Primary 20E25; Secondary 20D05, 20D20, 20E45, 20C20, 55R35.

Key words and phrases. Fusion, Sylow subgroups, finite simple groups, classifying spaces, modular representation theory.

The first author was partially supported by NSF DMS-1265587 and NSF DMS-0969009.

The second author was partially supported by UMR 7539 of the CNRS. 
Fusion also shows up in the hypothesis of the Cartan-Eilenburg Theorem on group cohomology. These appearances in both group theory and algebraic topology are precursors of things to come, as we will soon see.

Sometime in the 1990s, Luis Puig abstracted and formalized the study of fusion when he introduced the notion of a "fusion system", although Puig used different terminology. Roughly speaking, given a finite $p$-group $S$, a fusion system on $S$ is a category $\mathcal{F}$ whose objects are the subgroups of $S$, and such that for subgroups $P$ and $Q$ of $S$, the set $\operatorname{Hom}_{\mathcal{F}}(P, Q)$ of morphisms in $\mathcal{F}$ from $P$ to $Q$ is a set of group monomorphisms from $P$ into $Q$ satisfying two weak axioms. Here is the motivating example:

Standard Example. Let $G$ be a finite group, $p$ a prime, and $S$ a Sylow $p$-subgroup of $G$. Write $\mathcal{F}_{S}(G)$ for the fusion system on $S$ whose morphisms are those induced via conjugation in $G$. We call $\mathcal{F}_{S}(G)$ the $p$-fusion system of $G$.

We are most interested in the saturated fusion systems, which satisfy some additional axioms that are easily seen to be satisfied in the Standard Example using Sylow's Theorem.

If $\mathcal{F}$ is a fusion system of the form $\mathcal{F}_{S}(G)$ for some finite group $G$ and Sylow subgroup $S$ of $G$, then we say that $\mathcal{F}$ is realized by $G$. A saturated fusion system is said to be exotic if it is realized by no finite group. Exotic systems exist; indeed they seem to proliferate for large primes.

Puig introduced fusion systems as a tool in modular representation theory, the study of representations of finite groups $G$ over fields $F$ of prime characteristic $p$ dividing the order of $G$. In this case representations of $G$ are not completely reducible, leading one to consider the maximal direct sum decomposition of the group algebra of $G$ over $F$ into indecomposible subalgebras, called the blocks of $G$. Each block has an associated $p$-subgroup called the defect group of the block. Building on work of Alperin and Broue, Puig showed how to associate to each block a saturated fusion system on its defect group.

In the mid-1990s, Dave Benson, in Be2 and in an unpublished manuscript, began to suggest ways to associate classifying spaces to fusion systems. This was motivated in part by certain fusion patterns which Ron Solomon [So] had shown could not be realized by finite groups. Benson observed that certain spaces which could be constructed out of the Dwyer-Wilkerson space $B D I(4)$ [DW] had all of the properties that the classifying spaces of Solomon's "groups" would have had if they existed.

Somewhat later, several algebraic topologists including Carles Broto, Ran Levi, and Bob Oliver became interested in the Martino-Priddy Conjecture, which says roughly that the homotopy type of the $p$-completed classifying space $B G_{p}^{\wedge}$ of a finite group $G$ is determined by fusion in a Sylow $p$-subgroup $S$ of $G$. Given the notion of a fusion system, we can make such a statement precise: $B G_{p}^{\wedge}$ is homotopy equivalent to $B H_{p}^{\wedge}$ if and only if $\mathcal{F}_{S}(G) \cong \mathcal{F}_{T}(H)$ for $T \in \operatorname{Syl}_{p}(H)$.

In their work on the Martino-Priddy Conjecture, Broto, Levi, and Oliver were led to consider a second category $\mathcal{L}_{S}^{c}(G)$, related to $\mathcal{F}_{S}(G)$, called the centric linking system of $G$, and then to abstract this setup by considering pairs $(\mathcal{F}, \mathcal{L})$ where $\mathcal{F}$ is a saturated fusion system and $\mathcal{L}$ is a centric linking system associated to $\mathcal{F}$. Each such pair has a $p$-completed classifying space, and the classifying space of $\left(\mathcal{F}_{S}, \mathcal{L}_{S}^{c}(G)\right)$ is homotopy equivalent to $B G_{p}^{\wedge}$. Bob Oliver showed (in part via an appeal to the classification of the finite simple groups) that the abstract linking 
system associated to $\mathcal{F}_{S}(G)$ is unique, hence completing the proof of the MartinoPriddy Conjecture. Later work of Chermak [Ch], Oliver [O4], and Glauberman and Lynd $\mathrm{GbL}$ extended the result to the existence and uniqueness of linking systems for abstract saturated fusion systems, greatly simplifying the earlier proof, and then removed the dependence of the proof on the classification of the finite simple groups. This was the last step in confirming Benson's prediction about classifying spaces for fusion systems.

The study of fusion in finite groups is part of the local theory of finite groups. A $p$-local subgroup of $G$ is the normalizer of a nontrivial $p$-subgroup of $G$, and the local theory studies $G$ via its local subgroups. The local theory eventually became the most important tool in the proof of the theorem classifying the finite simple groups. It turns out that many notions in the local theory of groups can be translated into analogous notions for fusion systems, leading to a local theory of fusion systems. Indeed in some instances it is easier to work in the category of saturated fusion systems than in the category of groups. This leads to the hope that it may be possible to classify all simple 2-fusion systems and to use such a classification to simplify the classification of the finite simple groups.

The situation is made even more interesting by the existence of an infinite family of exotic simple 2-fusion systems, first encountered by Ron Solomon in a group theoretic context, conjectured to exist by David Benson in a topological context, and proved to exist by Levi and Oliver. But do there exist other exotic simple 2-fusion systems?

In short, fusion systems are of interest in at least three mathematical specialties: modular representation theory, algebraic topology, and local finite group theory. Moreover fusion systems can be regarded as either topological or algebraic objects and can be studied from either point of view. In this expository article we first give a brief introduction to the basic theory of fusion systems, including filling in the details of some definitions given only partially above. Our main reference for fusion systems is $\mathrm{AKO}$, although [Cr2 is also a good introduction to the subject. Then we go on to discuss various topics at greater length, including some raised earlier in this introduction, with emphasis on open problems in the theory of fusion systems, and applications of that theory in group theory and topology.

We close this introduction with a brief description of how the article is organized and some mention of certain of the topics.

In section 1 we give precise versions of some of the definitions alluded to above, introduce some of the most basic concepts, supply examples, and state and give references to the proofs of some fundamental results on fusion systems. The material in section 1 provides the foundation for later sections.

Section 2 supplies an introduction to a local theory of fusion systems, with an emphasis on saturated 2-fusion systems. Topics include a discussion in section 2.3 of the known simple 2-fusion systems. Section 2.4 outlines an approach for describing small extensions of simple systems tamely realized by the $p$-fusion system of some simple finite group. Section 2.6 describes a partition of simple 2-fusion systems into odd and even systems. Section 2.7 then supplies a sketchy outline of one possible approach to determining the odd simple systems and using that result to simplify part of the proof of the theorem classifying the finite simple groups.

Section 3 discusses the linking systems mentioned above, starting with the definitions in sections 3.1 and 3.2. As described in section 3.4, each saturated fusion 
system $\mathcal{F}$ possesses a unique centric linking system $\mathcal{L}$. However, despite this uniqueness, the linking system still encodes much information that is not readily apparent from the point of view of the fusion system. For example, in section 3.3 we encounter the notion of tameness, defined in terms of automorphisms of the linking system, that is useful in studying extensions of simple systems. Section 3.5 supplies a brief introduction to Chermak's theory of partial groups and localities, used in his proof of the existence and uniqueness of linking systems.

Section 4 discusses applications of fusion systems in algebraic topology. As we have seen, the existence of a linking system $\mathcal{L}$ allows us to associate a $p$-completed classifying space $|\mathcal{L}|_{p}^{\wedge}$ to each saturated $p$-fusion system, and the classifying space associated to $\mathcal{L}_{S}^{c}(G)$ is homotopy equivalent to $B G_{p}^{\wedge}$. Amongst other things this made it possible to reduce the Martino-Priddy Conjecture to a special case of the existence and uniqueness of linking systems, as discussed in section 4.3. In section 4.8, we see how to use this connection to show that the $p$-fusion systems of groups of Lie type over a field of order $q$ not divisible by $p$ are dependent not on $q$, but on the $p$-adic evaluation of a certain polynomial in $q$. On a slightly different theme, in section 4.7 we describe a characterization by Ragnarsson and Stancu of saturated fusion systems over a $p$-group $S$ in terms of certain idempotents in the endomorphism ring of the suspension spectrum of $B S$.

Section [5] discusses the role of fusion systems in modular representation theory. There is a brief introduction to block theory, followed by the construction of the fusion system of a block and some examples of how it influences its representation theory.

Finally, section 6 discusses some generalizations of fusion systems, where $S$ is replaced by some infinite $p$-group.

We end with a few remarks on notation. We always assume that $p$ is a fixed prime. Composition of homomorphisms (or of any other maps) is always taken from right to left. When $G$ is a group, $H \leq G$ is a subgroup, and $x \in G$, we write ${ }^{x} H=x H x^{-1}$ and $H^{x}=x^{-1} H x$, and similarly for ${ }^{x} g$ and $g^{x}$ when $g \in G$. As usual, for a finite group $G, O_{p}(G)$ and $O_{p^{\prime}}(G)$ are the largest normal $p$ - and $p^{\prime}$-subgroups, and $O^{p}(G)$ and $O^{p^{\prime}}(G)$ are the smallest normal subgroups of $p$-power index or $p^{\prime}$ index, respectively. Also, $C_{n}, D_{n}, Q_{n}$, and $S D_{n}$ denote cyclic, dihedral, (generalized) quaternion, and semidihedral groups of order $n$.

\section{BASIC PROPERTIES OF FUSION SYSTEMS}

We begin this section with the definitions of (saturated) fusion systems, followed by some of the other basic definitions and constructions: morphisms, kernels and quotients, normalizers and centralizers of $p$-subgroups, products, and normal fusion subsystems. Versions of Alperin's fusion theorem for fusion systems are then formulated, and the model theorem for constrained fusion systems is stated. A recent result of Benson, Grodal, and Henke is then described, stating very roughly that (at least at odd primes) a fusion system is detected by its restriction to elementary abelian subgroups. Finally, the section is ended with descriptions of several examples of fusion systems not realized by finite groups.

Most of the results stated in this section were originally formulated and proven by Puig in unpublished notes, and later published by him in $\mathrm{Pg} 4$ and/or $\mathrm{Pg} 5]$. Also, most of them can be found in Craven's book [Cr2. However, to avoid including 
too many references, in most cases we give only references in $\mathrm{AKO}$ (at least, when they are proven there), since its notation is closest to that used here.

1.1. Basic definitions. In general, when $G$ is a group and $H, K \leq G$, we write

$$
\operatorname{Hom}_{G}(H, K)=\left\{c_{g}=\left(h \mapsto{ }^{g} h\right) \in \operatorname{Hom}(H, K) \mid g \in G,{ }^{g} H \leq K\right\} .
$$

Using this notation, when $G$ is finite and $S \in \operatorname{Syl}_{p}(G), \mathcal{F}_{S}(G)$ is the category whose objects are the subgroups of $S$, and where for $P, Q \leq S$,

$$
\operatorname{Mor}_{\mathcal{F}_{S}(G)}(P, Q)=\operatorname{Hom}_{G}(P, Q) .
$$

Note that $\operatorname{Hom}_{G}(P, Q) \subseteq \operatorname{Inj}(P, Q)$, where $\operatorname{Inj}(H, K)$ is the set of injective group homomorphisms from $H$ to $K$.

With the help of this notation, we make the following definition.

Definition 1.1. A fusion system over a $p$-group $S$ is a category $\mathcal{F}$ such that

- $\mathrm{Ob}(\mathcal{F})=\{P \leq S \mid P$ a subgroup $\}$

- $\operatorname{Hom}_{S}(P, Q) \subseteq \operatorname{Hom}_{\mathcal{F}}(P, Q) \subseteq \operatorname{Inj}(P, Q)$ for each $P, Q \leq S$; and

- for each $P, Q \leq S$ and each $\varphi \in \operatorname{Hom}_{\mathcal{F}}(P, Q), \varphi^{-1} \in \operatorname{Hom}_{\mathcal{F}}(\varphi(P), P)$.

Thus each morphism in a fusion system $\mathcal{F}$ is an injective homomorphism of groups.

When $\mathcal{F}$ is a fusion system over $S$, and $P, Q \leq S$ and $x \in S$, we also write

$$
\begin{aligned}
\operatorname{Iso}_{\mathcal{F}}(P, Q) & =\left\{\varphi \in \operatorname{Hom}_{\mathcal{F}}(P, Q) \mid \varphi \text { is an isomorphism of groups }\right\}, \\
\operatorname{Aut}_{\mathcal{F}}(P) & =\operatorname{Iso}_{\mathcal{F}}(P, P) \leq \operatorname{Aut}(P), \\
\operatorname{Out}_{\mathcal{F}}(P) & =\operatorname{Aut}_{\mathcal{F}}(P) / \operatorname{Inn}(P) \leq \operatorname{Out}(P), \\
P^{\mathcal{F}} & =\left\{Q \leq S \mid \operatorname{Iso}_{\mathcal{F}}(P, Q) \neq \varnothing\right\}, \\
x^{\mathcal{F}} & =\left\{\varphi(x) \mid \varphi \in \operatorname{Hom}_{\mathcal{F}}(\langle x\rangle, S)\right\} .
\end{aligned}
$$

Clearly, $\operatorname{Iso}_{\mathcal{F}}(P, Q)=\operatorname{Hom}_{\mathcal{F}}(P, Q)$ if $|P|=|Q|$, and $\operatorname{Iso}_{\mathcal{F}}(P, Q)=\varnothing$ otherwise. By the third condition in Definition [1.1. each element of $\operatorname{Iso}_{\mathcal{F}}(P, Q)$ is an isomorphism (i.e., invertible) in the category $\mathcal{F}$.

In the above situation, by analogy with terminology for groups, we say that two subgroups $P, Q \leq S$ are $\mathcal{F}$-conjugate if $Q \in P^{\mathcal{F}}$ (i.e., if they are isomorphic in the category $\mathcal{F}$ ); and that two elements $x, y \in S$ are $\mathcal{F}$-conjugate if $y \in x^{\mathcal{F}}$.

In general, the concept of fusion systems as just defined is far too broad. To be of much use, we need to restrict attention to what are called "saturated" fusion systems. There are several equivalent definitions of this concept, of which the basic idea and earliest definition are due to Puig and which were formulated in the early 1990s (now called Frobenius categories in Pg4 and Pg5]).

The following definition is due to Roberts and Shpectorov [RS. Its details are not very important for following the rest of this article, but they help to give a feel for the conditions imposed on these objects.

Definition 1.2. Let $\mathcal{F}$ be a fusion system over a $p$-group $S$.

- A subgroup $P \leq S$ is called fully automized in $\mathcal{F}$ if $\operatorname{Aut}_{S}(P) \in \operatorname{Syl}_{p}\left(\operatorname{Aut}_{\mathcal{F}}(P)\right)$.

- A subgroup $P \leq S$ is called receptive in $\mathcal{F}$ if for each $Q \leq S$ and each $\varphi \in \operatorname{Iso}_{\mathcal{F}}(Q, P), \varphi$ extends to a homomorphism $\bar{\varphi} \in \operatorname{Hom}_{\mathcal{F}}\left(N_{\varphi}, S\right)$, where

$$
N_{\varphi}=\left\{x \in N_{S}(Q) \mid \varphi c_{x} \varphi^{-1} \in \operatorname{Aut}_{S}(P)\right\} .
$$


- The fusion system $\mathcal{F}$ is saturated if each subgroup of $S$ is $\mathcal{F}$-conjugate to a subgroup which is fully automized and receptive.

Note that, in the definition of a receptive subgroup, the defining condition for $N_{\varphi}$ is neccessary. If $\varphi$ does extend to some $\bar{\varphi} \in \operatorname{Hom}(N, S)$ for $N \leq N_{S}(Q)$, then $\varphi c_{x} \varphi^{-1}=c_{\bar{\varphi}(x)} \in \operatorname{Aut}_{S}(P)$ for each $x \in N$. So very roughly, the condition of $P$ being receptive means that $\mathcal{F}$-homomorphisms with image $P$ can be extended to the largest subgroup to which one could hope to extend them.

When $G$ is a finite group and $S \in \operatorname{Syl}_{p}(G)$, it is clear that $\mathcal{F}_{S}(G)$ (as defined above) is a fusion system over $S$. It is straightforward (though a bit tricky) to show, with the help of the Sylow theorems, that it is saturated.

1.2. Morphisms of fusion systems. Morphisms between fusion systems are defined as follows.

Definition 1.3. Let $\mathcal{F}$ and $\widetilde{\mathcal{F}}$ be fusion systems over $p$-groups $S$ and $\widetilde{S}$, respectively. A morphism from $\mathcal{F}$ to $\widetilde{\mathcal{F}}$ consists of a homomorphism $\alpha \in \operatorname{Hom}(S, \widetilde{S})$, together with a family of maps $\widehat{\alpha}_{P, Q}: \operatorname{Hom}_{\mathcal{F}}(P, Q) \longrightarrow \operatorname{Hom}_{\widetilde{\mathcal{F}}}(\alpha(P), \alpha(Q))$ for all $P, Q \leq S$, which satisfy the relation

$$
\widehat{\alpha}_{P, Q}(\varphi) \circ\left(\left.\alpha\right|_{P}\right)=\left(\left.\alpha\right|_{Q}\right) \circ \varphi \quad \text { for each } \varphi \in \operatorname{Hom}_{\mathcal{F}}(P, Q) .
$$

To simplify the notation, we denote such a morphism by the pair $(\alpha, \widehat{\alpha})$. Thus $\widehat{\alpha}$ represents the family $\left\{\widehat{\alpha}_{P, Q}\right\}_{P, Q \leq S}$. These maps can, in fact, be combined to form a functor $\widehat{\alpha}: \mathcal{F} \longrightarrow \widetilde{\mathcal{F}}$ : one which sends each object $P \leq S$ to its image $\alpha(P) \leq \widetilde{S}$.

Note that condition (11) implies that the maps $\widehat{\alpha}_{P, Q}$ are determined by $\alpha$. In other words, for each $\alpha \in \operatorname{Hom}(S, \widetilde{S})$, there is at most one $\widehat{\alpha}$ such that $(\alpha, \widehat{\alpha})$ is a morphism from $\mathcal{F}$ to $\widetilde{\mathcal{F}}$. The converse is not true: the maps $\widehat{\alpha}_{P, Q}$ do not determine $\alpha$ (as is easily seen, for example, when $\widetilde{S}$ is a group of order $p$ ).

The image of a morphism $(\alpha, \widehat{\alpha}): \mathcal{F} \longrightarrow \widetilde{\mathcal{F}}$ is the fusion system over $\operatorname{Im}(\alpha)$ where for each $P, Q \leq \operatorname{Im}(\alpha)$,

$$
\operatorname{Hom}_{\operatorname{Im}(\alpha, \widehat{\alpha})}(P, Q)=\widehat{\alpha}\left(\operatorname{Hom}_{\mathcal{F}}\left(\alpha^{-1}(P), \alpha^{-1}(Q)\right)\right) \leq \operatorname{Inj}(P, Q) .
$$

Such a morphism is surjective if $\operatorname{Im}(\alpha, \widehat{\alpha})=\widetilde{\mathcal{F}}$; equivalently, if $\alpha$ is surjective, and $\widehat{\alpha}_{P, Q}$ is surjective for all $P, Q$ containing $\operatorname{Ker}(\alpha)$. It can be shown that if $\alpha$ is injective or $\mathcal{F}$ is saturated, then $\operatorname{Im}(\alpha, \widehat{\alpha})$ is a subsystem of $\mathcal{F}$ and $(\alpha, \widehat{\alpha}): \mathcal{F} \longrightarrow \operatorname{Im}(\alpha, \widehat{\alpha})$ is a surjective morphism of fusion systems. Finally, $(\alpha, \widehat{\alpha})$ is an isomorphism if it is surjective, and $\alpha$ (and hence all of the maps $\widehat{\alpha}_{P, Q}$ ) are injective. One easily sees that under this definition, isomorphisms of fusion systems are invertible.

When $\alpha \in \operatorname{Iso}(S, \widetilde{S})$ extends to an isomorphism $(\alpha, \widehat{\alpha}): \mathcal{F} \stackrel{\cong}{\longrightarrow} \widetilde{\mathcal{F}}$, we say that $\alpha$ is fusion preserving (with respect to the fusion systems $\mathcal{F}$ and $\widetilde{\mathcal{F}}$ ). Thus $\mathcal{F}$ and $\widetilde{\mathcal{F}}$ are isomorphic as fusion systems if there is a fusion preserving isomorphism $\alpha \in \operatorname{Iso}(S, \widetilde{S})$. An automorphism $\alpha \in \operatorname{Aut}(S)$ is fusion preserving (with respect to $\mathcal{F}$ ) if it extends to some (unique) automorphism $(\alpha, \widehat{\alpha})$ of $\mathcal{F}$. It is convenient to identify automorphisms of $\mathcal{F}$ with fusion preserving automorphisms of $S$, and we write

$\operatorname{Aut}(\mathcal{F})=\{\alpha \in \operatorname{Aut}(S) \mid \alpha$ is fusion preserving with respect to $\mathcal{F}\}$, $\operatorname{Out}(\mathcal{F})=\operatorname{Aut}(\mathcal{F}) / \operatorname{Aut}_{\mathcal{F}}(S)$. 
As one might expect, each homomorphism between finite groups induces a morphism between their fusion systems at any given prime. We leave the following proposition as an easy exercise.

Proposition 1.4. Let $f: G \longrightarrow H$ be a homomorphism of finite groups. Fix $S \in \operatorname{Syl}_{p}(G)$ and $T \in \operatorname{Syl}_{p}(H)$ such that $f(S) \leq T$. Then $f$ induces a morphism of fusion systems $(\alpha, \widehat{\alpha}): \mathcal{F}_{S}(G) \longrightarrow \mathcal{F}_{T}(H)$, where $\alpha=\left.f\right|_{S} \in \operatorname{Hom}(S, T)$; and where $\widehat{\alpha}$ sends conjugation by $g \in G$ to conjugation by $f(g) \in H$.

If $f$ is surjective, then $(\alpha, \widehat{\alpha})$ is surjective. If $f$ is surjective and $\operatorname{Ker}(f)$ has order prime to $p$, then $(\alpha, \widehat{\alpha})$ is an isomorphism of fusion systems.

For example, if $K \unlhd G$ is a normal subgroup of order prime to $p$ and $S \in \operatorname{Syl}_{p}(G)$, then $\mathcal{F}_{S}(G)=\mathcal{F}_{S}(G / K)$, where we identify $S$ with $S K / K \in \operatorname{Syl}_{p}(G / K)$. Thus fusion systems ignore all normal subgroups of order prime to $p$.

1.3. Strongly closed subgroups, kernels, and quotients. Once we have defined morphisms between fusion systems, it is natural to look at their kernels. If $(\alpha, \widehat{\alpha}): \mathcal{F} \longrightarrow \widetilde{\mathcal{F}}$ is a morphism and $T=\operatorname{Ker}(\alpha)$, then one could try to look at the fusion system over $T$ consisting of all morphisms in $\mathcal{F}$ which are sent to the identity morphism of the trivial subgroup in $\widetilde{\mathcal{F}}$. However, one quickly sees that this is the subcategory consisting of all $\mathcal{F}$-morphisms between subgroups of $T$, which almost never is a saturated fusion system. Since there does not seem to be any other good way to define a kernel as a fusion subsystem, we look instead at the subgroup $\operatorname{Ker}(\alpha)$.

Definition 1.5. Let $\mathcal{F}$ be a fusion system over a $p$-group $S$. A subgroup $T \leq S$ is strongly closed in $\mathcal{F}$ if no element of $T$ is $\mathcal{F}$-conjugate to an element of $S \backslash T$; i.e., if $T$ is a union of $\mathcal{F}$-conjugacy classes of elements.

Equivalently, in the above situation, $T \leq S$ is strongly closed if for each $P \leq T$ and each $\varphi \in \operatorname{Hom}_{\mathcal{F}}(P, S), \varphi(P) \leq T$. In particular, if $T$ is strongly closed in $\mathcal{F}$, then $T$ must be normal in $S$.

The following proposition is an easy exercise.

Proposition 1.6. For any morphism $(\alpha, \widehat{\alpha}): \mathcal{F} \longrightarrow \widetilde{\mathcal{F}}$ of fusion systems, $\operatorname{Ker}(\alpha)$ is strongly closed in $\mathcal{F}$.

The converse of Proposition 1.6 is also true: each subgroup strongly closed in $\mathcal{F}$ is the kernel (in the above sense) of some morphism defined on $\mathcal{F}$-a morphism between saturated fusion systems if $\mathcal{F}$ is saturated. This requires the definition of quotient fusion systems $\mathcal{F} / T$ for $T$ strongly closed in $\mathcal{F}$.

When $T \unlhd S$ is strongly closed in $\mathcal{F}$, define $\mathcal{F} / T$ to be the fusion system over $S / T$ where for $P / T, Q / T \leq S / T$,

$$
\operatorname{Hom}_{\mathcal{F} / T}(P / T, Q / T)=\left\{(x T \mapsto \varphi(x) T) \in \operatorname{Hom}(P / T, Q / T) \mid \varphi \in \operatorname{Hom}_{\mathcal{F}}(P, Q)\right\} .
$$

Note that for all $\varphi \in \operatorname{Mor}(\mathcal{F})$ between subgroups containing $T, \varphi(T)=T$ since $T$ is strongly closed, and hence $(x T \mapsto \varphi(x) T)$ is well defined. By [AKO, Lemma II.5.4] or [O1, Lemma 2.6], if $\mathcal{F}$ is saturated, then so is $\mathcal{F} / T$.

By examination of the above definition, one sees that in fact, $\mathcal{F} / T=N_{\mathcal{F}}(T) / T$ for any $T \unlhd S$ strongly closed in $\mathcal{F}$. (See Definition 1.8 for the definition of the normalizer fusion system $N_{\mathcal{F}}(T)$.) This is analogous to the Frattini argument for groups: if $H \unlhd G$ and $T \in \operatorname{Syl}_{p}(H)$, then $G=H N_{G}(T)$ (see [A2, (6.2)]), and 
hence $G / H \cong N_{G}(T) / N_{H}(T)$. Since $N_{H}(T) / T$ has order prime to $p$, the fusion systems of $G / H$ and $N_{G}(T) / T$ are isomorphic. Thus in this situation (and if $\left.T \leq S \in \operatorname{Syl}_{p}(G)\right)$,

$$
\mathcal{F}_{S}(G) / T=\mathcal{F}_{S / T}\left(N_{G}(T) / T\right)=\mathcal{F}_{S / T}(G / H) .
$$

By analogy with the standard result for groups, each morphism $(\alpha, \widehat{\alpha}): \mathcal{F} \longrightarrow \widetilde{\mathcal{F}}$ of fusion systems induces an isomorphism

$$
\mathcal{F} / \operatorname{Ker}(\alpha) \cong \operatorname{Im}(\alpha, \widehat{\alpha}) .
$$

In particular, $\operatorname{Im}(\alpha, \widehat{\alpha})$ is always saturated if $\mathcal{F}$ is, since $\mathcal{F} / \operatorname{Ker}(\alpha)$ is saturated.

In order to show that each strongly closed subgroup is the kernel of some morphism of fusion systems, one must also construct an appropriate morphism from $\mathcal{F}$ to $\mathcal{F} / T$. This is more subtle than it looks at first glance.

Theorem 1.7 (Cr2, Theorem 5.14], AKO, Theorem II.5.12]). For each saturated fusion system $\mathcal{F}$ over a p-group $S$, and each subgroup $T \unlhd S$ strongly closed in $\mathcal{F}$, there is a surjective morphism of fusion systems $(\alpha, \widehat{\alpha}): \mathcal{F} \longrightarrow \mathcal{F} / T$ where $\alpha: S \longrightarrow S / T$ is the natural surjection.

When $T$ is normal in $\mathcal{F}$, i.e., when $\mathcal{F}=N_{\mathcal{F}}(T)$, then it is easy to construct a morphism from $\mathcal{F}$ to $\mathcal{F} / T$. But when $T$ is only assumed to be strongly closed in $\mathcal{F}$, the construction is much less obvious. Since $T$ is strongly closed in $\mathcal{F}$, each morphism $\varphi \in \operatorname{Hom}_{\mathcal{F}}(P, Q)$ induces a homomorphism

$$
\widetilde{\varphi}: P T / T \cong P /(P \cap T) \longrightarrow Q /(Q \cap T) \cong Q T / T \text {. }
$$

But since $\varphi$ need not extend to a morphism in $\operatorname{Hom}_{\mathcal{F}}(P T, Q T)$, there is no easy reason why $\widetilde{\varphi}$ should lie in $\operatorname{Hom}_{\mathcal{F} / T}(P T / T, Q T / T)$.

In Cr2, $\S 5.2]$, Craven gives several "isomorphism theorems" for quotient fusion systems: theorems analogous to the standard isomorphism theorems for groups. We list two of them here [Cr2, Propositions 5.16, 5.18], for a saturated fusion system $\mathcal{F}$ over a $p$-group $S$ :

- If $\mathcal{E} \leq \mathcal{F}$ is a saturated fusion subsystem over $R \leq S$, then $\mathcal{E} Q / Q \cong$ $\mathcal{E} /(Q \cap R)$. Here, $\mathcal{E} Q / Q$ is the image of $\mathcal{E}$ under the morphism $\mathcal{F} \longrightarrow \mathcal{F} / Q$ of Theorem 1.7 .

- If $Q \leq R \leq S$ and $Q$ and $R$ are both strongly closed in $\mathcal{F}$, then $(\mathcal{F} / Q) /(R / Q) \cong$ $\mathcal{F} / R$.

1.4. Normalizers and centralizers of subgroups. Normalizers and centralizers of subgroups in fusion systems are defined in a very natural way.

Definition 1.8. Let $\mathcal{F}$ be a fusion system over the $p$-group $S$, and let $T \leq S$ be any subgroup. Then $C_{\mathcal{F}}(T) \leq N_{\mathcal{F}}(T) \leq \mathcal{F}$ are the fusion subsystems over $C_{S}(T)$ and $N_{S}(T)$, respectively, defined as follows:

$$
\begin{aligned}
& \operatorname{Hom}_{C_{\mathcal{F}}(T)}(P, Q)=\left\{\varphi \in \operatorname{Hom}_{\mathcal{F}}(P, Q)\left|\exists \bar{\varphi} \in \operatorname{Hom}_{\mathcal{F}}(P T, Q T), \bar{\varphi}\right|_{P}=\varphi,\left.\bar{\varphi}\right|_{T}=\operatorname{Id}_{T}\right\}, \\
& \operatorname{Hom}_{N_{\mathcal{F}}(T)}(P, Q)=\left\{\varphi \in \operatorname{Hom}_{\mathcal{F}}(P, Q)\left|\exists \bar{\varphi} \in \operatorname{Hom}_{\mathcal{F}}(P T, Q T), \bar{\varphi}\right|_{P}=\varphi, \bar{\varphi}(T)=T\right\} .
\end{aligned}
$$

This does fit with our intuitive idea of what a normalizer or centralizer of $T$ should be: each morphism should extend to one which sends $T$ to itself or is the identity on $T$, respectively. However, there is one problem with the definition: $N_{\mathcal{F}}(T)$ and $C_{\mathcal{F}}(T)$ need not be saturated, not even when $\mathcal{F}$ is saturated.

To get around this, two more definitions are needed. 
Definition 1.9. Let $\mathcal{F}$ be a fusion system over a $p$-group $S$.

- A subgroup $T \leq S$ is fully normalized in $\mathcal{F}$ if $\left|N_{S}(T)\right| \geq\left|N_{S}(P)\right|$ for each $P \in T^{\mathcal{F}}$.

- A subgroup $T \leq S$ is fully centralized in $\mathcal{F}$ if $\left|C_{S}(T)\right| \geq\left|C_{S}(P)\right|$ for each $P \in T^{\mathcal{F}}$.

By a result of Roberts and Shpectorov [RS, $\S 4-5]$ (see also [AKO, Lemma I.2.6(c)]), these subgroups can be characterized as follows.

Proposition 1.10. If $\mathcal{F}$ is a satured fusion system over a $p$-group $S$, then for each $T \leq S$,

- $T$ is fully centralized in $\mathcal{F}$ if and only if it is receptive in $\mathcal{F}$; and

- $T$ is fully normalized in $\mathcal{F}$ if and only if it is fully automized and receptive in $\mathcal{F}$.

We now get the following theorem:

Theorem 1.11 ([Pg4, Proposition 2.15], Cr2, Theorem 4.8], [KK, Theorem I.5.5]). Let $\mathcal{F}$ be a saturated fusion system over a $p$-group $S$. Then for each subgroup $T \leq S$,

- $N_{\mathcal{F}}(T)$ is saturated if $T$ is fully normalized in $\mathcal{F}$, while

- $C_{\mathcal{F}}(T)$ is saturated if $T$ is fully centralized in $\mathcal{F}$.

Once normalizers and centralizers have been defined, it is natural to define normal and central subgroups.

Definition 1.12. Let $\mathcal{F}$ be a fusion system over a $p$-group $S$.

- A subgroup $T \leq S$ is normal in $\mathcal{F}$ (written $T \unlhd \mathcal{F}$ ) if $N_{\mathcal{F}}(T)=\mathcal{F}$.

- A subgroup $T \leq S$ is central in $\mathcal{F}$ if $C_{\mathcal{F}}(T)=\mathcal{F}$.

- Let $O_{p}(\mathcal{F}) \unlhd S$ be the largest subgroup of $S$ which is normal in $\mathcal{F}$.

- Let $Z(\mathcal{F}) \unlhd S$ be the largest subgroup of $S$ which is central in $\mathcal{F}$.

It follows directly from the definitions that if $T$ and $U$ are both normal in $\mathcal{F}$ (central in $\mathcal{F}$ ), then so is $T U$. Hence it does make sense to talk about the largest normal and central subgroups. When $\mathcal{F}$ is saturated, it is not hard to see that (by analogy with centers in groups) $Z(\mathcal{F})$ is the group of all $x \in S$ such that $x^{\mathcal{F}}=\{x\}$.

We now turn to the special case where $\mathcal{F}=\mathcal{F}_{S}(G)$ for some finite group $G$ with $S \in \operatorname{Syl}_{p}(G)$. Normalizers and centralizers in $\mathcal{F}$ are straightforward: for each $T \leq S$

- $T$ is fully normalized in $\mathcal{F}$ if and only if $N_{S}(T) \in \operatorname{Syl}_{p}\left(N_{G}(T)\right)$, in which case $N_{\mathcal{F}}(T)=\mathcal{F}_{N_{S}(T)}\left(N_{G}(T)\right)$; and

- $T$ is fully centralized in $\mathcal{F}$ if and only if $C_{S}(T) \in \operatorname{Syl}_{p}\left(C_{G}(T)\right)$, in which case $C_{\mathcal{F}}(T)=\mathcal{F}_{C_{S}(T)}\left(C_{G}(T)\right)$

(see [AKO, Proposition I.5.4]). Also, $Z(\mathcal{F})=Z\left(G / O_{p^{\prime}}(G)\right)$ in this situation (where we identify $S$ with a Sylow $p$-subgroup of $\left.G / O_{p^{\prime}}(G)\right)$. This is the $Z^{*}$-theorem: shown by Glauberman [Gl1] when $p=2$, and following from the classification of finite simple groups [GLS3, Remark 7.8.3] when $p$ is odd. Thus there is a connection between the center of a group and that of its fusion system, but only by a deep theorem or a case-by-case check.

As for $O_{p}(\mathcal{F})$, there is no simple relationship in general. When $G$ is finite, $S \in \operatorname{Syl}_{p}(G)$, and $Q \unlhd S, Q$ is normal in $\mathcal{F}_{S}(G)$ if and only if $\mathcal{F}_{S}(G)=N_{\mathcal{F}_{S}(G)}(Q)$, 
and we just saw that $N_{\mathcal{F}_{S}(G)}(Q)=\mathcal{F}_{S}\left(N_{G}(Q)\right)$. Thus $Q$ is normal in $\mathcal{F}_{S}(G)$ whenever $N_{G}(Q)$ controls $p$-fusion in $G$. For example, when $p=2, G=A_{5}$, and $S \in \operatorname{Syl}_{2}(G), S \unlhd \mathcal{F}_{S}(G)$, while $S$ is certainly not normal in $G$.

When $\mathcal{F}$ is a saturated fusion system over $S$ and $T \leq S$, the following implications follow directly from the definitions:

$$
T \text { central in } \mathcal{F} \Longrightarrow T \text { normal in } \mathcal{F} \Longrightarrow T \text { strongly closed in } \mathcal{F} \text {. }
$$

Simple examples (involving fusion systems of groups) show that neither of these implications is an equivalence.

1.5. Fusion systems generated by a set of homomorphisms. It follows easily from the definition that an intersection of fusion systems over a given $p$-group $S$ is again a fusion system over $S$. (This is not true for saturated fusion systems.) Hence it makes sense to talk about the fusion system over $S$ generated by a set of morphisms between subgroups of $S$, or another fusion system and a set of morphisms, etc. For example, if $\mathcal{F}_{0}$ is a fusion system over $S, P_{1}, \ldots, P_{k} \leq S$ are subgroups, and $\Gamma_{i} \leq \operatorname{Aut}\left(P_{i}\right)$ for each $i$, then

$$
\mathcal{F}=\left\langle\mathcal{F}_{0}, \Gamma_{1}, \ldots, \Gamma_{k}\right\rangle
$$

means that $\mathcal{F}$ is the smallest fusion system over $S$ which contains $\mathcal{F}_{0}$, and such that $\operatorname{Aut}_{\mathcal{F}}\left(P_{i}\right) \geq \Gamma_{i}$ for each $i$. More concretely, for $P, Q \leq S, \operatorname{Hom}_{\mathcal{F}}(P, Q)$ is the set of composites of restrictions of morphisms in $\mathcal{F}_{0}$ and of automorphisms in the $\Gamma_{i}$. In general, of course, one does not expect a fusion system defined in this way to be saturated, but we give one example below (Proposition 1.29) of conditions which are sufficient to imply this.

For example, this concept of "the fusion system generated by" is useful when defining products of fusion systems. Assume $\mathcal{F}$ and $\widetilde{\mathcal{F}}$ are fusion systems over $p$ groups $S$ and $\widetilde{S}$, respectively. For each $\varphi \in \operatorname{Mor}(\mathcal{F})$ and $\widetilde{\varphi} \in \operatorname{Mor}(\widetilde{\mathcal{F}})$, we can regard $\varphi \times \widetilde{\varphi}$ as a morphism between subgroups of $S \times \widetilde{S}$. So it makes sense to define

$$
\mathcal{F} \times \widetilde{\mathcal{F}}=\langle\operatorname{Mor}(\mathcal{F}) \times \operatorname{Mor}(\widetilde{\mathcal{F}})\rangle:
$$

the smallest fusion system over $S \times \widetilde{S}$ which contains all such products of morphisms $\varphi \times \widetilde{\varphi}$. If $\mathcal{F}$ and $\widetilde{\mathcal{F}}$ are both saturated, then $\mathcal{F} \times \widetilde{\mathcal{F}}$ is also saturated (see, e.g., AKO, Theorem I.6.6]).

1.6. Alperin's fusion theorem. Alperin's fusion theorem fits nicely into the above context: it says that each saturated fusion system is generated by automorphisms of certain of its subgroups. There are different versions of this result, depending on how far one wants to restrict this family of subgroups.

Definition 1.13. Let $\mathcal{F}$ be a saturated fusion system over a $p$-group $S$.

- A subgroup $P \leq S$ is $\mathcal{F}$-centric if for each $Q \in P^{\mathcal{F}}, C_{S}(Q) \leq Q$.

- A subgroup $P \leq S$ is $\mathcal{F}$-radical if $O_{p}\left(\operatorname{Out}_{\mathcal{F}}(P)\right)=1$; equivalently, each normal $p$-subgroup of $\operatorname{Aut}_{\mathcal{F}}(P)$ is contained in $\operatorname{Inn}(P)$.

One of the simplest forms of Alperin's fusion theorem is the following.

Theorem 1.14 ([BLO2, Theorem A.10]). If $\mathcal{F}$ is a satuated fusion system over a finite p-group $S$, then $\mathcal{F}$ is generated by the groups $\operatorname{Aut}_{\mathcal{F}}(P)$ for subgroups $P \leq S$ which are $\mathcal{F}$-centric, $\mathcal{F}$-radical, and fully normalized in $\mathcal{F}$.

Equivalently, each morphism in $\mathcal{F}$ is a composite of restrictions of $\mathcal{F}$-automorphisms of subgroups which are $\mathcal{F}$-centric, $\mathcal{F}$-radical, and fully normalized. 
The second version of the statement is closer to the original form of Alperin's theorem Alp1.

Corollary 1.15. Let $\mathcal{F}$ be a saturated fusion system over a p-group $S$. Then $\mathcal{F}=\mathcal{F}_{S}(S)$ if and only if $\operatorname{Aut}_{\mathcal{F}}(P)$ is a p-group for each $\mathcal{F}$-centric subgroup $P \leq S$.

Proof. If $\operatorname{Aut}_{\mathcal{F}}(P)$ is a $p$-group for each $\mathcal{F}$-centric subgroup $P \leq S$, then $\operatorname{Aut}_{\mathcal{F}}(P)=$ $\operatorname{Aut}_{S}(P)$ for each $\mathcal{F}$-centric subgroup $P \leq S$ which is fully automized (Definition 1.2), and in particular, for each $\mathcal{F}$-centric subgroup which is fully normalized (Proposition 1.10). So by Theorem 1.14, $\mathcal{F}$ is generated by groups $\operatorname{Aut}_{S}(P)$ for certain $P \leq S$, and hence $\mathcal{F}=\mathcal{F}_{S}(S)$. The converse is clear.

When $G$ is a finite group, and $S \in \operatorname{Syl}_{p}(G)$ is abelian, then by Burnside's fusion theorem $\mathrm{Bu}, \S 123], N_{G}(S)$ controls $p$-fusion in $G$ (i.e., $\mathcal{F}_{S}(G)=\mathcal{F}_{S}\left(N_{G}(S)\right)$ ). The analogous result for fusion systems is an easy consequence of Theorem 1.14.

Corollary 1.16. If $\mathcal{F}$ is a saturated fusion system over a abelian p-group $S$, then $S \unlhd \mathcal{F}$, and $\mathcal{F}=\mathcal{F}_{S}\left(S \rtimes \operatorname{Aut}_{\mathcal{F}}(S)\right)$.

Proof. Since $S$ is abelian, no proper subgroup of $S$ can be $\mathcal{F}$-centric. So by Theorem 1.14. every morphism in $\mathcal{F}$ is the restriction of some $\varphi \in \operatorname{Aut}_{\mathcal{F}}(S)$.

This is a special case of what Stancu calls resistant p-groups. A $p$-group $S$ is resistant if it is normal in each saturated fusion system over $S$. Thus Corollary 1.16 says that all abelian $p$-groups are resistant. In St], Stancu shows that all extraspecial $p$-groups of order at least $p^{4}$, and all metacyclic $p$-groups when $p$ is odd, are resistant. Other examples of resistant $p$-groups were listed by Sambale Sa], Craven and Glesser [CG], Xu [Xu, and other authors.

As another simple application of Theorem 1.14, we list all saturated fusion systems over dihedral, quaternion, and semidihedral 2-groups.

Example 1.17. Let $S$ be a group of order $2^{k}$ with presentation

$$
S=\left\langle a, b \mid a^{2^{k-1}}=1, b^{2}=z_{0},(a b)^{2}=z_{1}\right\rangle,
$$

where $z_{0}, z_{1} \in\left\{a^{2^{k-2}}, 1\right\}$ are fixed, $k \geq 3$, and $k \geq 4$ if $z_{0} \neq 1$ or $z_{1} \neq 1$. Thus $S$ is dihedral if $z_{0}=z_{1}=1, S$ is quaternion if $z_{0}=z_{1}=a^{2^{k-2}}$, and $S$ is semidihedral if $z_{0} \neq z_{1}$. (Note that $b a b^{-1}=a^{-1} z_{0} z_{1}$ and $a b a^{-1}=z_{0} z_{1} a^{2} b$ in all cases.) For each $i \in \mathbb{Z}$, define

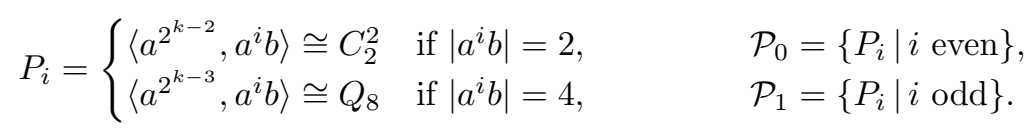

Then each of $\mathcal{P}_{0}$ and $\mathcal{P}_{1}$ is a conjugacy class of subgroups of $S$. For each $I \subseteq\{0,1\}$, set

$$
\left.\mathcal{F}_{I}=\langle\operatorname{Aut}(P)| P \in \mathcal{P}_{i} \text {, some } i \in I\right\rangle
$$

as a fusion system over $S$. In all of these fusion systems $\mathcal{F}_{I}, \operatorname{Aut}_{\mathcal{F}}(P)=\operatorname{Aut}_{S}(P)$ for each $P \leq S$ not in $\bigcup_{i \in I} \mathcal{P}_{i}$. Of these four fusion systems, $\mathcal{F}_{\{0\}} \cong \mathcal{F}_{\{1\}}$ when $S$ is dihedral or quaternion, so there are just three distinct systems up to isomorphism in those cases. All of these fusion systems are realizable as fusion systems of finite groups. 
Proof. The key observation is that in each case, the subgroups $P \in \mathcal{P}_{0} \cup \mathcal{P}_{1}$ are the

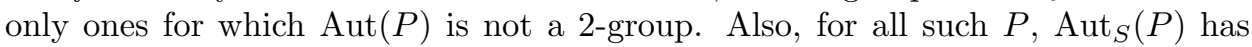
index 3 in $\operatorname{Aut}(P)$, so $\operatorname{Aut}_{\mathcal{F}}(P)$ must be equal to one of these two groups. Since the subgroups in each $\mathcal{P}_{i}$ are all $S$-conjugate to each other, they must all have the same group of $\mathcal{F}$-automorphisms, and thus there are only four choices. By Theorem 1.14, a saturated fusion system is determined by these automorphism groups.

None of the above remarks explain why the fusion systems $\mathcal{F}_{I}$ are all saturated, and the simplest way to show that is to check that they are all fusion systems of finite groups. For example, the three fusion systems over a dihedral group $S$ are realized by $S, P G L_{2}(q)$, and $P S L_{2}\left(q^{2}\right)$ for an appropriate odd prime power $q$ depending on $k$. (We can take $q=3$ or 9 when $k=3$ or 4 , respectively.) When $S$ is semidihedral, for some choice of $q \equiv 3(\bmod 4)$, the four fusion systems are realized by $S, G L_{2}(q), S L_{3}(q)$, and a certain extension of $P S L_{2}\left(q^{2}\right)$ by a product of a field and diagonal automorphism.

Once one knows that each saturated fusion system $\mathcal{F}$ is generated by automorphism groups of certain subgroups, it is natural to try to determine a minimal set of such subgroups whose automorphisms generate $\mathcal{F}$. This was done for groups by Goldschmidt [Gd1], and a similar result holds for fusion systems.

Definition 1.18. - If $G$ is a finite group and $H<G$ is a proper subgroup, $H$ is strongly $p$-embedded in $G$ if $p|| H \mid$, and for $x \in G \backslash H, p \nmid\left|H \cap{ }^{x} H\right|$.

- If $\mathcal{F}$ is a saturated fusion system over a finite $p$-group $S$, a subgroup $P<S$ is $\mathcal{F}$-essential if $P$ is $\mathcal{F}$-centric and fully normalized in $\mathcal{F}$, and $\operatorname{Out}_{\mathcal{F}}(P)$ contains a strongly $p$-embedded subgroup.

For example, if $G$ is such that each pair of distinct Sylow $p$-subgroups of $G$ intersects trivially (and there is more than one Sylow $p$-subgroup), then the normalizer of a Sylow $p$-subgroup is strongly $p$-embedded in $G$. If each Sylow $p$-subgroup of $G$ is cyclic or (if $p=2$ ) generalized quaternion, then the normalizer of a subgroup of order $p$ is strongly $p$-embedded in $G$ (if it is a proper subgroup). In topological terms, a finite group $G$ has a strongly $p$-embedded subgroup if and only if the poset of nontrivial $p$-subgroups of $G$ is nonconnected.

For groups, Alperin's fusion theorem was first formulated in terms of essential subgroups by Goldschmidt Gd1. The analogue for fusion systems of his result is a stronger version of Theorem 1.14, which was originally shown by Puig.

Theorem 1.19 (Alperin-Goldschmidt Fusion Theorem [AKO, Theorem I.3.5]). Let $\mathcal{F}$ be a saturated fusion system over a p-group $S$. Then

$$
\left.\mathcal{F}=\left\langle\operatorname{Aut}_{\mathcal{F}}(S), \operatorname{Aut}_{\mathcal{F}}(P)\right| P<S \text { is } \mathcal{F} \text {-essential }\right\rangle .
$$

In fact, in the situation of Theorem 1.19] it suffices to take one subgroup from each $\mathcal{F}$-conjugacy class of $\mathcal{F}$-essential subgroups.

The following proposition helps to explain the importance of strongly embedded subgroups in Theorem 1.19. It also shows why any generating set for $\mathcal{F}$ must include automorphisms from at least one representative in each $\mathcal{F}$-conjugacy class containing $\mathcal{F}$-essential subgroups.

Proposition 1.20 ([AKO, Proposition I.3.3]). Let $\mathcal{F}$ be a saturated fusion system over a p-group $S$, and let $P<S$ be a proper subgroup which is $\mathcal{F}$-centric and fully normalized in $\mathcal{F}$. Let $H \leq \operatorname{Aut}_{\mathcal{F}}(P)$ be the subgroup generated by all elements 
$\varphi \in \operatorname{Aut}_{\mathcal{F}}(P)$ which extend to $\mathcal{F}$-homomorphisms between strictly larger subgroups of $S$. Then either

- $H=\operatorname{Aut}_{\mathcal{F}}(P)$ and $P$ is not $\mathcal{F}$-essential; or

- $H<\operatorname{Aut}_{\mathcal{F}}(P), H / \operatorname{Inn}(P)$ is strongly $p$-embedded in $\operatorname{Out}_{\mathcal{F}}(P)$, and $P$ is $\mathcal{F}$-essential.

1.7. Normal fusion subsystems and simple fusion systems. The definition of a normal fusion subsystem is more complicated, and more subtle.

Definition 1.21. A saturated fusion subsystem $\mathcal{E} \leq \mathcal{F}$ over a subgroup $T \leq S$ is normal if

- $T$ is strongly closed in $\mathcal{F}$;

- (invariance condition) each $\alpha \in \operatorname{Aut}_{\mathcal{F}}(T)$ is fusion preserving with respect to $\mathcal{E}$ (i.e., extends to $(\alpha, \widehat{\alpha}) \in \operatorname{Aut}(\mathcal{E}))$;

- (Frattini condition) for each $P \leq T$ and each $\varphi \in \operatorname{Hom}_{\mathcal{F}}(P, T)$, there are $\alpha \in \operatorname{Aut}_{\mathcal{F}}(T)$ and $\varphi_{0} \in \operatorname{Hom}_{\mathcal{E}}(P, T)$ such that $\varphi=\alpha \circ \varphi_{0}$; and

- (extension condition) each $\alpha \in \operatorname{Aut}_{\mathcal{E}}(T)$ extends to some $\bar{\alpha} \in \operatorname{Aut}_{\mathcal{F}}\left(T C_{S}(T)\right.$ ) such that $\left[\bar{\alpha}, C_{S}(T)\right] \leq Z(T)$.

For example, if $G$ is a finite group, $S \in \operatorname{Syl}_{p}(G), H \unlhd G$ is a normal subgroup, and $T=H \cap S \in \operatorname{Syl}_{p}(H)$, then $\mathcal{F}_{T}(H)$ is a normal fusion subsystem of $\mathcal{F}_{S}(G)$ (see, e.g., AKO, Proposition I.6.2]). However, there can be normal subsystems of $\mathcal{F}_{S}(G)$ which do not come from normal subgroups of $G$. This is discussed in more detail in section 2.1 .

The Frattini condition is motivated by the Frattini argument: if $G$ is a finite group, $H \unlhd G$, and $T \in \operatorname{Syl}_{p}(H)$, then $G=H \cdot N_{G}(T)$ (see [A2, (6.2)]). The Frattini and invariance conditions in the above definition can be replaced by other, equivalent conditions which are closer to the definition of normal subgroups of a finite group (see, e.g., AKO, Proposition I.6.4]), but which require other technicalities in their formulations.

The following example helps to explain the importance of the extension condition in Definition 1.21. Set $p=3$, and set $G=G_{1} \times G_{2}$ where $G_{1} \cong G_{2}$ are symmetric groups of order 6 . Fix $S_{i} \in \operatorname{Syl}_{3}\left(G_{i}\right)$, and set $S=S_{1} \times S_{2}$. Let $G_{0}<G$ be the subgroup of index 2 which contains neither $G_{1}$ nor $G_{2}$. Set $\mathcal{F}=\mathcal{F}_{S}\left(G_{0}\right)$ and $\mathcal{E}=\mathcal{F}_{S_{1}}\left(G_{1}\right)$. Then $\mathcal{E} \leq \mathcal{F}, S_{1}$ is strongly closed in $\mathcal{F}$, and the invariance and Frattini conditions are easily seen to hold. However, the extension condition does not hold, since for $g \in G_{1}$ of order $2, c_{g} \in \operatorname{Aut}_{G_{1}}\left(S_{1}\right)$ does not extend to an element $\bar{\alpha} \in \operatorname{Aut}_{G_{0}}(S)$ such that $[\bar{\alpha}, S] \leq S_{1}$. Note that in this example, there is no normal subgroup of $H \unlhd G_{0}$ which realizes the fusion subsystem $\mathcal{E}$. The extension condition ensures that when $G$ is a constrained group (see section 1.8), each normal subsystem of its fusion system is the fusion system of a unique normal subgroup of $G$.

Once normal subsystems have been defined, the definition of a simple fusion system is clear: a saturated fusion system is simple if it contains no proper nontrivial normal fusion subsystems.

As usual, when $H$ is a group and $\alpha \in \operatorname{Aut}(H)$, we set $[\alpha, H]=\left\langle h^{-1} \alpha(h) \mid h \in H\right\rangle$. (This is the commutator subgroup in the usual sense when $\alpha$ is considered as an element of $H \rtimes \operatorname{Aut}(H)$.) 
Definition-Proposition 1.22 ([Cr2, Propositions 7.45, 7.46, and 7.48]). Let $\mathcal{F}$ be a saturated fusion system over a $p$-group $S$, and define

$$
\begin{aligned}
\mathfrak{f o c}(\mathcal{F}) & =\left\langle x y^{-1} \mid x, y \in S, y \in x^{\mathcal{F}}\right\rangle, \\
\mathfrak{h} \mathfrak{y} \mathfrak{p}(\mathcal{F}) & =\left\langle[\alpha, P] \mid P \leq S, \alpha \in O^{p}\left(\operatorname{Aut}_{\mathcal{F}}(P)\right)\right\rangle .
\end{aligned}
$$

Then $\mathfrak{f o c}(\mathcal{F})=\mathfrak{h} \mathfrak{y} \mathfrak{p}(\mathcal{F})[S, S]$, and $\mathfrak{f o c}(\mathcal{F})$ and $\mathfrak{h} \mathfrak{y} \mathfrak{p}(\mathcal{F})$ are both strongly closed in $\mathcal{F}$. Also, $\mathfrak{h y p}(\mathcal{F})$ is the smallest subgroup $T$ of $S$ strongly closed in $\mathcal{F}$ such that $\mathcal{F} / T=\mathcal{F}_{S / T}(S / T)$.

The definition of $\mathfrak{h y p}(\mathcal{F})$ was motivated by analogous definitions for a group. If $\mathcal{F}=\mathcal{F}_{S}(G)$ where $G$ is a finite group and $S \in \operatorname{Syl}_{p}(G)$, then $\mathfrak{f o c}(\mathcal{F})=S \cap[G, G]$ by the focal subgroup theorem of Higman, and $\mathfrak{h y p}(\mathcal{F})=S \cap O^{p}(G)$ by the hyperfocal subgroup theorem of Puig [Pg3]. (See also [Cr2, Theorems 1.30 and 1.33]).

We are now ready to describe, for saturated fusion systems, the normal subsystems analogous to the subgroups $O^{p}(G)$ and $O^{p^{\prime}}(G)$ when $G$ is a finite group.

Theorem 1.23 ([BCGLO2, Theorems 4.3 and 5.4]). The following hold for each saturated fusion system $\mathcal{F}$ over a p-group $S$.

(a) There is a unique normal subsystem $O^{p}(\mathcal{F}) \unlhd \mathcal{F}$ over $\mathfrak{h y p}(\mathcal{F})$ such that $\operatorname{Aut}_{O^{p}(\mathcal{F})}(P) \geq O^{p}\left(\operatorname{Aut}_{\mathcal{F}}(P)\right)$ for each $P \leq \mathfrak{h y p}(\mathcal{F})$. Furthermore,

$$
O^{p}(\mathcal{F})=\mathcal{F} \Longleftrightarrow \mathfrak{h y p}(\mathcal{F})=S \Longleftrightarrow \mathfrak{f o c}(\mathcal{F})=S .
$$

(b) There is a unique smallest normal subsystem $O^{p^{\prime}}(\mathcal{F}) \unlhd \mathcal{F}$ over $S$.

For the last statement in (a), we refer to [AKO, Corollary I.7.5].

1.8. The model theorem. A saturated fusion system $\mathcal{F}$ over a $p$-group $S$ is called constrained if there is a normal subgroup $Q \unlhd \mathcal{F}$ which is also $\mathcal{F}$-centric. Since $Q$ is normal in $\mathcal{F}$, the centricity condition just means that $C_{S}(Q) \leq Q$ (since $Q^{\mathcal{F}}=\{Q\}$ ). Clearly, $\mathcal{F}$ is constrained if and only if $O_{p}(\mathcal{F})$ is $\mathcal{F}$-centric.

A model for a constrained saturated fusion system $\mathcal{F}$ over $S$ is a finite group $G$ such that $S \in \operatorname{Syl}_{p}(G), \mathcal{F}_{S}(G)=\mathcal{F}$, and $C_{G}\left(O_{p}(G)\right) \leq O_{p}(G)$. The model theorem says that all constrained fusion systems have models, unique up to isomorphism.

Theorem 1.24 (The model theorem AKO, Theorem I.4.9]). Let $\mathcal{F}$ be a constrained, saturated fusion system over a p-group $S$. Then $\mathcal{F}$ has a model, which is unique up to isomorphism of groups.

More generally, let $Q \unlhd \mathcal{F}$ be a normal, $\mathcal{F}$-centric subgroup. Let $G$ be any finite group such that $S \in \operatorname{Syl}_{p}(G), Q \unlhd G, C_{G}(Q) \leq Q$, and $\operatorname{Aut}_{G}(Q)=\operatorname{Aut}_{\mathcal{F}}(Q)$. Then $G$ is a model for $\mathcal{F}$ and, in particular, $\mathcal{F}=\mathcal{F}_{S}(G)$.

In fact, this theorem is shown in $\mathrm{AKO}$ in a much more rigid form: with stronger conditions on the isomorphism between two models.

As one example, if $\mathcal{F}$ is an arbitrary saturated fusion system over $S$, and $P \leq S$ is $\mathcal{F}$-centric and fully normalized, then $N_{\mathcal{F}}(P)$ is a constrained fusion system since $P$ is normal and centric. The model theorem says that $N_{\mathcal{F}}(P)$ is always realizable, even when $\mathcal{F}$ is not.

We will see in section 2(e.g., in Lemma 2.33) the important role which the model theorem plays in the local theory of fusion systems. We also refer to [DGMP1, DGMP2 for examples of how it can be used when generalizing theorems about groups to theorems about fusion systems. 
1.9. Detection by elementary abelian $p$-groups. The following result was proven by Benson, Grodal, and Henke, and is of a very different nature from most theorems about fusion systems shown so far.

Theorem 1.25 ([BGH, Theorem B] $)$. Let $S$ be a p-group. Let $\mathcal{P}$ be the set of elementary abelian subgroups of $S$ (if $p$ is odd) or the set of abelian subgroups of exponent at most 4 (if $p=2$ ). Assume $\mathcal{E} \leq \mathcal{F}$ are two saturated fusion systems over $S$, with the property that $\operatorname{Hom}_{\mathcal{E}}(P, Q)=\operatorname{Hom}_{\mathcal{F}}(P, Q)$ for each $P, Q \in \mathcal{P}$. Then $\mathcal{E}=\mathcal{F}$.

When $p$ is odd, Theorem 1.25 can be combined with classical results of Quillen Q to show that if $H \leq G$ are finite groups, where $|G: H|$ is prime to $p$, and the restriction map $H^{*}\left(G ; \mathbb{F}_{p}\right) \longrightarrow H^{*}\left(H ; \mathbb{F}_{p}\right)$ is an isomorphism, or more generally an $F$-isomorphism in the sense of Quillen, then $H$ controls $p$-fusion in $G$ (i.e., $\mathcal{F}_{S}(H)=\mathcal{F}_{S}(G)$ for $\left.S \in \operatorname{Syl}_{p}(H) \subseteq \operatorname{Syl}_{p}(G)\right)$. Here, a homomorphism $f: R \longrightarrow S$ of commutative (or graded commutative) $\mathbb{F}_{p}$-algebras is an $F$-isomorphism (see Q, §3]) if it is an "isomorphism modulo nilpotent elements"; i.e., each element in $\operatorname{Ker}(f)$ is nilpotent, and for each $s \in S$, there is $n$ such that $s^{p^{n}} \in \operatorname{Im}(f)$.

In particular, this generalizes a theorem of Mislin (but only at odd primes) which says that $H$ controls fusion in $G$ if $H^{*}\left(G ; \mathbb{F}_{p}\right) \cong H^{*}\left(H ; \mathbb{F}_{p}\right)$. We refer to [BGH, Theorem A] for more details.

1.10. Analogies between groups and fusion systems. One can look to finite group theory for intuition about fusion systems. In particular, one can seek analogies in the category of saturated fusion systems of basic concepts in group theory. We have already discussed fusion system analogues of normal subgroups (in section 1.7) and factor groups (in section 1.3). In this section we consider a few more analogies, and explore how well such analogies are preserved by our functor $G \mapsto \mathcal{F}_{S}(G)$ from groups to fusion systems.

Let $\mathcal{F}$ be a saturated fusion system on a finite $p$-group, and let $G$ be a finite group with Sylow $p$-subgroup $S$. In Definition 1.21 we defined the notion of a normal subsystem of $\mathcal{F}$. From Example 2.1, if $H \unlhd G$, then $\mathcal{F}_{S \cap H}(H) \unlhd \mathcal{F}_{S}(G)$. However not all normal subsystems of $\mathcal{F}_{S}(G)$ correspond to normal subgroups of $G$.

Example 1.26. Let $G$ be simple with $S$ cyclic of order greater than $p$. (For example take $G \cong P S L_{2}(8)$ and $p=3$.) Let $T$ be the subgroup of $S$ of order $p$. Then $\mathcal{F}_{S}(G)=\mathcal{F}_{S}\left(N_{G}(S)\right)$, so $\mathcal{F}_{T}(T)$ is a proper normal subsystem of $\mathcal{F}_{S}(G)$ that does not correspond to any normal subgroup of $G$.

On the other hand if $C_{G}\left(O_{p}(G)\right) \leq O_{p}(G)$, then from Theorem II.7.5 in [AKO], the map $H \mapsto \mathcal{F}_{S \cap H}(H)$ is a bijection between the set of all normal subgroups of $G$ and the set of all normal subsystems of $\mathcal{F}_{S}(G)$. The extension condition from Definition 1.21 is included in the definition of a "normal subsystem" precisely to ensure this correspondence exists.

Let $\alpha: G \rightarrow X$ be a surjective group homomorphism. If $H \unlhd G$, then $\alpha(H) \unlhd X$, and if $Y \unlhd X$, then the preimage $\alpha^{-1}(Y)$ of $Y$ is a normal subgroup of $G$.

On the other hand suppose $(\alpha, \hat{\alpha}): \mathcal{F} \rightarrow \mathcal{X}$ is a surjective morphism of fusion systems (cf. section 1.2 ). If $\mathcal{E} \unlhd \mathcal{F}$, then by 8.9 in [A3], $\alpha(\mathcal{E}) \unlhd \mathcal{X}$. But if $\mathcal{Y} \unlhd \mathcal{X}$, then the preimage of $\mathcal{Y}$ need not be normal in $\mathcal{F}$. 
Example 1.27. Let $L$ be a simple group with abelian Sylow $p$-subgroup $T, V$ a faithful $\mathbb{F}_{p} L$-module, and $G$ the semidirect product of $V$ by $L$ with respect to this representation. Take $S=V T \in \operatorname{Syl}_{p}(G)$ and $\alpha: G \rightarrow L$ to be the homomorphism $l v \mapsto l$. Let $\mathcal{X}=\mathcal{F}_{T}(L)$ and $\mathcal{F}=\mathcal{F}_{S}(G)$. Then $\alpha$ induces a surjective morphism $(\alpha, \hat{\alpha}): \mathcal{F} \rightarrow \mathcal{X}$. Now $T$ is normal in $\mathcal{X}$ but the inverse image $S$ of $T$ under $\alpha$ is not normal in $\mathcal{F}$, as can be seen from the bijection mentioned above.

There are several equivalent definitions of the notion of a solvable group. For example, $G$ is solvable if and only if its derived series descends to 1 . And $G$ is solvable if and only if all its composition factors are of prime order.

In Definition 2.18 the Puig series for $\mathcal{F}$ is defined; one can think of the Puig series for a saturated fusion system as the analogue of the derived series of a group. Let $\mathcal{F}^{\infty}$ be the last term in the Puig series of $\mathcal{F}$, and define $\mathcal{F}$ to be Puig solvable if $\mathcal{F}^{\infty}=1$. It turns out (cf. II.12.4 in $[\mathrm{AKO}$ ) that $\mathcal{F}$ is Puig solvable if and only if $\mathcal{F}$ is constrained and a model for $\mathcal{F}$ is $p$-solvable. Thus Puig solvability is one possible analogue for fusion systems of solvability for groups.

In section II.10 of $\mathrm{AKO}$ there appears a definition of composition series and composition factors for $\mathcal{F}$; these notions are not quite as well behaved as the corresponding group theoretic notions, but there is a Jordan-Holder Theorem. This allows us to define a second notion of solvability for fusion systems. As this notion is weaker than Puig solvability, we will refer to it as "weak solvability". Thus $\mathcal{F}$ is weakly solvable if all its composition factors are "of order $p$ ": that is isomorphic to the fusion system of the group of order $p$. It develops (cf. II.12.7 in [AKO]) that if $\mathcal{F}$ is weakly solvable, then $\mathcal{F}$ is constrained, so it possesses a model, but that model need not be $p$-solvable. An example of a weakly solvable system that is not Puig solvable is the system $\mathcal{F}_{S}(G)$ appearing in Example 1.27. Hence there are at least two possible definitions of a solvable fusion system, and these definitions are not equivalent.

We complete this section with an example of two group theoretic concepts for which we currently have no satisfactory analogue in the theory of fusion systems.

Recall for $H \leq G$ that $N_{G}(H), C_{G}(H)$ denote the normalizer, centralizer of $H$ in $G$, respectively. If $S$ is Sylow in $\mathcal{F}$ and $P \leq S$, then the normalizer and centralizer $N_{\mathcal{F}}(P)$ and $C_{\mathcal{F}}(P)$ of $P$ in $\mathcal{F}$ are defined in Definition 1.8 Viewing $P$ as the subsystem $\mathcal{F}_{P}(P)$ of $\mathcal{F}$, we then have normalizers and centralizers of such subsystems. But we do not know how to define the normalizer or centralizer of an arbitrary (saturated) subsystem of $\mathcal{F}$.

What properties should the centralizer $C_{\mathcal{F}}(\mathcal{E})$ of a subsystem $\mathcal{E}$ of $\mathcal{F}$ possess? First, a Sylow group $C_{S}(\mathcal{E})$ of $C_{\mathcal{F}}(\mathcal{E})$ should centralize $\mathcal{E}$ : that is we should have $\mathcal{E} \leq C_{\mathcal{F}}\left(C_{S}(\mathcal{E})\right)$. Indeed $C_{S}(\mathcal{E})$ should be the largest such subgroup of $S$. Going further, $\mathcal{F}$ should contain a central product $\mathcal{E} * C_{\mathcal{F}}(\mathcal{E})$ of $\mathcal{E}$ with $C_{\mathcal{F}}(\mathcal{E})$, as defined in section 2.1, and $C_{\mathcal{F}}(\mathcal{E})$ should be the largest such subsystem of $\mathcal{F}$.

Perhaps the best we can hope to achieve is to define the normalizer and/or centralizer of $\mathcal{E}$ when $\mathcal{E}$ is suitably embedded in $\mathcal{F}$; in particular at a minimum we may wish to require that a Sylow group of $\mathcal{E}$ be fully normalized in $\mathcal{F}$.

If $\mathcal{E} \unlhd \mathcal{F}$, then $C_{\mathcal{F}}(\mathcal{E})$ is defined and normal in $\mathcal{F}$ by Theorem 4 in $\mathrm{A} 4$. Problem 7.4 asks if it is possible to define the normalizer and/or centralizer in $\mathcal{F}$ for members of some larger class of subsystems of $\mathcal{F}$.

So in the end the examples in this section seem to say that many (if not most) of the fundamental notions in finite group theory have analogues in the category 
of saturated fusion systems, but that the analogies are not always all that well behaved. One can use the intuition developed in working with finite groups to suggest and prove results on fusion systems, but one must take care when working with corresponding notions when the analogy is inexact. Of more interest are those cases where one can take advantage of differences in behavior to prove some fact in one category, and then use that proof to say something that is not all that apparent in the second category. This is the sort of leverage that the program to classify simple 2-fusion systems and finite groups discussed in the latter part of section 2 seeks to exploit.

1.11. Examples of fusion systems. We have already described how Alperin's fusion theorem can be used to list all saturated fusion systems over dihedral, semidihedral, and quaternion groups. We now look at some other ways to construct saturated fusion systems; in particular, some which are "exotic" in the sense of not being fusion systems of any finite group.

Example 1.28. Fix a prime $p \geq 5$ and a prime power $q$ such that $p \mid(q-1)$ but $p^{2} \nmid(q-1)$. Set $G=P S L_{p}(q)$, and fix $S \in \operatorname{Syl}_{p}(G)$. Let $D<G$ be the subgroup of classes of diagonal matrices, and set $N=N_{G}(D)$. Let $P_{0} \cong C_{p} \times C_{p}$ be generated by the classes of $\operatorname{diag}\left(1, \zeta, \zeta^{2}, \ldots, \zeta^{p-1}\right)$ (for $\zeta \in \mathbb{F}_{q}^{\times}$of order $p$ ) and the matrix for the cyclic permutation $(123 \cdots p)$. Let $\mathcal{P}_{0}$ be its $G$-conjugacy class, and let $\mathcal{P}_{1}, \ldots, \mathcal{P}_{p-1}$ be the other $G$-conjugacy class of subgroups which are conjugate to $P_{0}$ in $P G L_{p}(q)$. For each subset $I \subseteq\{0,1, \ldots, p-1\}$, define

$$
\left.\mathcal{F}_{I}=\left\langle\mathcal{F}_{S}(N), \operatorname{Aut}_{G}(P)\right| P \in \mathcal{P}_{i} \text { for some } i \in I\right\rangle .
$$

The idea when constructing the $\mathcal{F}_{I}$ is to "mix" the fusion systems of $G$ and $N$. For example, for $0 \leq i \leq p-1$ and $P \in \mathcal{P}_{i}$, $\operatorname{Aut}_{\mathcal{F}_{I}}(P)=\operatorname{Aut}_{G}(P)$ if $i \in I$ and $\operatorname{Aut}_{\mathcal{F}_{I}}(P)=\operatorname{Aut}_{N}(P)$ otherwise. Also, $\mathcal{F}_{I}=\mathcal{F}_{S}(G)$ if $I=\{0,1, \ldots, p-1\}$, and $\mathcal{F}_{I}=\mathcal{F}_{S}(N)$ if $I=\varnothing$. As shown in [BLO2, Example 9.3], $\mathcal{F}_{I}$ is saturated for all $I$ and is exotic if $0<|I|<p$.

The next proposition provides one tool which is useful in many cases when directly constructing small saturated fusion systems, including many which are exotic. To state it, we need to define two more properties of a $p$-subgroup $P$ of a finite group $G$ :

- $P$ is $p$-centric in $G$ if $Z(P) \in \operatorname{Syl}_{p}\left(C_{G}(P)\right)$; and

- $P$ is essential in $G$ if $P$ is $p$-centric in $G$ and $\operatorname{Out}_{G}(P)$ has a strongly $p$-embedded subgroup.

It is not hard to see that if $P \leq S \in \operatorname{Syl}_{p}(G)$, then $P$ is $p$-centric in $G$ if and only if it is $\mathcal{F}_{S}(G)$-centric (Definition 1.13).

Proposition 1.29 ([BLO4, Proposition 5.1]). Fix a finite group $G$ and $S \in \operatorname{Syl}_{p}(G)$. Let $R_{1}, \ldots, R_{m}<S$ be subgroups such that no $R_{i}$ is $G$-conjugate to a subgroup of $R_{j}$ for any $j \neq i$. For each $i$, set $K_{i}=\operatorname{Out}_{G}\left(R_{i}\right)$, and fix a subgroup $\Delta_{i} \leq \operatorname{Out}\left(R_{i}\right)$ which contains $K_{i}$. Assume for each $i$ that

(1) $R_{i}$ is p-centric in $G$, but no proper subgroup $P<R_{i}$ is $\mathcal{F}$-centric or an essential $p$-subgroup of $G$; and

(2) $K_{i}$ is strongly $p$-embedded in $\Delta_{i}$.

Set $\mathcal{F}=\left\langle\mathcal{F}_{S}(G), \Delta_{1}, \ldots, \Delta_{m}\right\rangle$; then $\mathcal{F}$ is a saturated fusion system over $S$. 
Other constructions similar to Proposition 1.29 have been obtained by Onofrei On.

Note, in the situation of Proposition [1.29, that when $\mathcal{F}>\mathcal{F}_{S}(G), P$ can be $p$-centric in $G$ but not $\mathcal{F}$-centric. Also, the condition $p \nmid\left[\Delta_{i}: K_{i}\right]$ assumed in [BLO4, Proposition 5.1] follows from (2).

To illustrate the use of Proposition [1.29, we describe some examples of fusion systems over extraspecial groups of order $p^{3}$, which were classified by Ruiz and Viruel in [RV]. Assume $p$ is odd, and let $S$ be a nonabelian group of order $p^{3}$ and exponent $p$. Then $S / Z(S) \cong C_{p}^{2}$, and each automorphism of $S$ which is the identity modulo $Z(S)$ is inner. So $\operatorname{Out}(S) \cong G L_{2}(p)$. Let $\mathcal{H}$ be the set of subgroups of index $p$ in $S$; then $|\mathcal{H}|=p+1$ and each $Q \in \mathcal{H}$ is isomorphic to $C_{p}^{2}$.

TABLE 1

\begin{tabular}{|c|c|c|c|}
\hline$p$ & $\operatorname{Out}_{\mathcal{F}}(S)$ & $\operatorname{Aut}_{\mathcal{F}}(Q)(Q \in \mathcal{H})$ & $\operatorname{group}(\mathrm{s})$ \\
\hline \hline 3 & $S D_{16}$ & $G L_{2}(3)(4)$ & $J_{4}, R u,{ }^{2} F_{4}(2)$ \\
\hline 7 & $\mathbb{F}_{7}^{\times} \succ C_{2}$ & $S L_{2}(7) .2(6)$ & $F i_{24}$ \\
\hline 7 & $\mathbb{F}_{7}^{\times} \succ C_{2}$ & $S L_{2}(7) .2(6), G L_{2}(7)(2)$ & exotic \\
\hline 7 & $\mathbb{F}_{49}^{\times} \rtimes C_{2}$ & $S L_{2}(7) .2(8)$ & exotic \\
\hline 13 & $\mathbb{F}_{13}^{\times} \times C_{2} \Sigma_{4}$ & $S L_{2}(13) .4(6)$ & $F_{1}=M$ \\
\hline
\end{tabular}

Example 1.30. In Table 1, $S$ is always a nonabelian (extraspecial) group of order $p^{3}$ and exponent $p$, and $\mathcal{H}$ is the set of subgroups of index $p$. Also $S L_{2}(p)$.n (for $n \mid(p-1))$ denotes the unique subgroup of $G L_{2}(p)$ which contains $S L_{2}(p)$ with index $n$. Each row describes one possible saturated fusion system over $S$, and indicates whether it is realized by a finite group or is exotic. (These are just a few of the many possible fusion systems; a complete list is given in [RV].) Thus, for example, the second entry in the table refers to a fusion system $\mathcal{F}$ over $S$ where $\operatorname{Out}_{\mathcal{F}}(S)$ is the group of monomial matrices in $G L_{2}(7)$ (which acts on $\mathcal{H}$ with orbits $\mathcal{H}_{1}$ and $\mathcal{H}_{2}$ of length 6 and 2); $\operatorname{Aut}_{\mathcal{F}}(Q) \cong S L_{2}(7) .2$ for $Q \in \mathcal{H}_{1}$, and $Q$ is not radical for $Q \in \mathcal{H}_{2}$. Also, this is isomorphic to the fusion system of the almost simple sporadic group $F i_{24}$.

In each case, $\mathcal{F}$ can be constructed using Proposition 1.29, applied with $G=$ $S \rtimes \operatorname{Out}_{\mathcal{F}}(S)$, and with $\left\{R_{1}, \ldots, R_{m}\right\}$ a set of representatives for the $\operatorname{Out}_{\mathcal{F}}(S)$-orbits in $\mathcal{H}$ which are listed in the third column. For each $i=1, \ldots, m, \Delta_{i} \leq G L_{2}(p)$ is the group given in the third row for that orbit, and one can check that $K_{i} \stackrel{\text { def }}{=}$ $\operatorname{Aut}_{G}\left(R_{i}\right)=N_{\Delta_{i}}\left(\operatorname{Aut}_{S}\left(R_{i}\right)\right.$ ) (hence is strongly $p$-embedded in $\left.\Delta_{i}\right)$. Condition (1) in the proposition is easily seen to hold.

Other examples of exotic fusion systems at odd primes are constructed in BLO2, $\S 9]$, BLO4, § 5], $\mathrm{CP}$, [DRV], [PS], [Rz, O5], and [CrOS]. In particular, in O5] and [CrOS], large families of exotic simple fusion systems are constructed over $p$ groups with abelian subgroup of index $p$ : enough examples to suggest that there might even be a sense in which "almost all" simple saturated fusion systems over a $p$-group for odd $p$ are exotic. 
In most cases, the hardest parts of the construction is showing that they are saturated and showing that they are exotic. In fact, the only tool we have so far (when $p$ is odd) for proving a saturated fusion system is exotic is the classification of finite simple groups. In all examples constructed until now, one shows that $\mathcal{F}$ is exotic by first showing that if it is realizable, it is realizable by a finite simple (or almost simple) group. Afterwards, one has to go down the list of simple groups, and check whether the $p$-fusion system of any of them could be isomorphic to $\mathcal{F}$.

1.12. The Solomon fusion systems. The following examples are the only currently known exotic simple fusion systems over 2-groups and also the only currently known fusion systems which can be proven to be exotic without recourse to the classification of finite simple groups.

Example 1.31. Let $q$ be an odd prime power. Set $G=\operatorname{Spin}_{7}(q)$, and choose $S \in \operatorname{Syl}_{2}(G)$. Let $x \in Z(G) \leq S$ be the generator. There is a saturated fusion system $\operatorname{Sol}(q)$ over $S$ such that $C_{\operatorname{Sol}(q)}(x)=\mathcal{F}_{S}(G)$, and such that all involutions in $S$ are conjugate in $\operatorname{Sol}(q)$.

We refer to [LO] and [AC] for two different constructions of $\operatorname{Sol}(q)$ : the first by defining a generating set of automorphism groups, and the second as the fusion system of a certain (infinite) amalgamated free product of finite groups. Note that $|S|=2^{3 m+1}$, where $m=v_{2}\left(q^{2}-1\right)$ (the 2 -adic valuation of $\left.q^{2}-1\right)$. The fusion system of $\operatorname{Spin}_{7}(q)$ depends up to isomorphism only on $v_{2}\left(q^{2}-1\right.$ ) (see Theorem 4.3(c)), and hence $\operatorname{Sol}(q)$ as constructed in [LO or AC depends up to isomorphism only on $v_{2}\left(q^{2}-1\right)$. In fact, when $q \equiv \pm 3(\bmod 8)$ and hence $|S|=2^{10}$, it follows from [OV2, Theorem 7.8] that there is up to isomorphism a unique saturated fusion system, independent of $q$, satisfying the conditions listed above.

The motivation for these fusion systems comes from work of Ron Solomon. In [So, Theorem 3.2], he showed that when $q \equiv \pm 3(\bmod 8)$, there is no finite group $G$ such that all involutions in $G$ are conjugate and the centralizer of an involution has the fusion system of $\operatorname{Spin}_{7}(q)$. As an immediate consequence, $\operatorname{Sol}(q)$ is exotic in this case. When $q \equiv \pm 1(\bmod 8)$ (i.e., when $\left.v_{2}\left(q^{2}-1\right) \geq 4\right)$, the same result, together with a theorem of Goldschmidt again implies (with a little more work) that $\operatorname{Sol}(q)$ is exotic. We refer to the proof of [LO, Proposition 3.4] for details. So in these cases, there is a proof that the fusion systems are exotic which is independent of the classification of finite simple groups.

The Solomon fusion systems will be discussed again in section 2(e.g., in Example 2.11 and Corollary 2.16), and in sections 4.4 and 6.1

\section{A LOCAL THEORY OF FUSION SYSTEMS}

Let $G$ be a finite group and $p$ a prime. A p-local subgroup of $G$ is the normalizer of a nontrivial $p$-subgroup of $G$. The local theory of finite groups studies $G$ from the point of view of its local subgroups. The local theory was the most important tool in the classification of the finite simple groups. Parts of that theory can be translated into a local theory of saturated fusion systems. In particular there is some hope that simple 2-fusion systems can be classified, and that a suitable local theory of fusion systems can be used to simplify the proof of the theorem classifying the finite simple groups.

Interspersed with an introduction to some of the basic concepts underlying the local theory of fusion systems is a discussion of the known simple 2-fusion systems, 
and discussion of "small extensions" of simple systems. Eventually we outline a program of the first author aimed at, first, classifying a large subclass of the class of simple 2-fusion systems, and then, second, using the result on fusion systems to simplify the proof of the theorem classifying the finite simple groups.

2.1. Some basics. Let $\mathcal{F}$ be a saturated fusion system on a finite $p$-group $S$. Recall from Definition 1.21 that we have a notion of a normal subsystem of $\mathcal{F}$. This allows us to define a saturated system $\mathcal{F}$ to be simple if $\mathcal{F}$ has no proper nontrivial normal subsystems. To get some feeling for how the two notions of "normality" (for groups and for fusion systems) compare, consider the following example:

Example 2.1. If $\mathcal{F}=\mathcal{F}_{S}(G)$ is the $p$-fusion system of $G$ and $H \unlhd G$, then by I.6.2 in $\mathrm{AKO}, \mathcal{F}_{S \cap H}(H) \unlhd \mathcal{F}$. However the converse need not be true: there may be normal subsystems of $\mathcal{F}$ that do not correspond to normal subgroups of $G$.

Thus, in some respects, normal subsystems of fusion systems behave like normal subgroups of groups, but there are significant differences. For example, suppose $\mathcal{E}_{i} \unlhd \mathcal{F}$ on $T_{i}$ for $i=1,2$. Then $\mathcal{E}_{1} \cap \mathcal{E}_{2}$ is a subsystem of $\mathcal{F}$ satisfying the invariance and Frobenius conditions of Definition 1.21, but it need not be saturated and hence need not be normal. However there exists a normal subsystem $\mathcal{E}_{1} \wedge \mathcal{E}_{2}$ of $\mathcal{F}$ on $T_{1} \cap T_{2}$ that is the largest normal subsystem of $\mathcal{F}$ normal in $\mathcal{E}_{1}$ and $\mathcal{E}_{2}$ (cf II.9.1 in $\mathrm{AKO}$ ). It follows that for each subgroup $P$ of $S$ there is a smallest normal subsystem $\mathcal{E}$ of $\mathcal{F}$ whose Sylow group contains $P$. For if $\mathcal{E}_{1}$ and $\mathcal{E}_{2}$ are two such minimal systems, then $\mathcal{E}_{1}=\mathcal{E}_{1} \wedge \mathcal{E}_{2}=\mathcal{E}_{2}$.

Write $[P]_{\mathcal{F}}$ for this system, and call it the normal closure of $P$ in $\mathcal{F}$.

Example 2.2. Recall from Theorem 1.23 that there is a smallest normal subsystem $O^{p^{\prime}}(\mathcal{F})$ of $\mathcal{F}$ on $S$. Indeed $O^{p^{\prime}}(\mathcal{F})$ is the normal closure of $S$ in $\mathcal{F}$.

Similarly from Theorem 1.23 there is a unique normal subsystem $O^{p}(\mathcal{F})$ of $\mathcal{F}$ on the hyperfocal subgroup $\mathfrak{h y p}(\mathcal{F})$ of $\mathcal{F}$ such that for each $U \leq \mathfrak{h} \mathfrak{n p}(\mathcal{F})$, we have $O^{p}\left(\operatorname{Aut}_{\mathcal{F}}(U)\right) \leq \operatorname{Aut}_{O^{p}(\mathcal{F})}(U)$. Moreover $\mathcal{F} / \mathfrak{h y p}(\mathcal{F}) \cong \mathcal{F}_{S / \mathfrak{h y p}(\mathcal{F})}(S / \mathfrak{h y p}(\mathcal{F}))$ is the $p$-fusion system of a $p$-group.

Example 2.3. If $\mathcal{F}=\mathcal{F}_{S}(G)$, then $O^{p}(\mathcal{F})=\mathcal{F}_{S \cap O^{p}(G)}\left(O^{p}(G)\right)$, where $O^{p}(G)$ is the smallest normal subgroup $H$ of $G$ such that $G / H$ is a $p$-group.

Recall from section 1.5 that given systems $\mathcal{F}_{i}$ on $S_{i}, 1 \leq i \leq n$, one can form the direct product $\mathcal{F}=\mathcal{F}_{1} \times \cdots \times \mathcal{F}_{n}$ on $S=S_{1} \times \cdots \times S_{n}$. If each $\mathcal{F}_{i}$ is saturated, then so is $\mathcal{F}$. Then if $D \leq Z(\mathcal{F})$ with $D \cap Z\left(\mathcal{F}_{i}\right)=1$ for each $1 \leq i \leq n$, we can form $\mathcal{F}^{+}=\mathcal{F} / D$, and $\mathcal{F}^{+}=\mathcal{F}_{1}^{+} * \cdots * \mathcal{F}_{n}^{+}$is a central product of the images $\mathcal{F}_{i}^{+} \cong \mathcal{F}_{i}$ of the $\mathcal{F}_{i}$; that is, for distinct $i$ and $j, \mathcal{F}_{i}^{+}$"commutes" with $\mathcal{F}_{j}^{+}$.

In general centralizers and normalizers of subsystems of a saturated fusion system $\mathcal{F}$ are not defined. However in certain situations one can define such systems. In particular if $\mathcal{E} \unlhd \mathcal{F}$, then the centralizer $C_{\mathcal{F}}(\mathcal{E})$ is defined and normal in $\mathcal{F}$, and $\mathcal{F}$ contains a central product $\mathcal{E} * C_{\mathcal{F}}(\mathcal{E})$ of the two subsystems as a normal subsystem (cf. II.9.3 and II.9.4 in [AKO] ). For example the Sylow subgroup $C_{S}(\mathcal{E})$ of $C_{\mathcal{F}}(\mathcal{E})$ is the largest subgroup $X$ of $S$ with $\mathcal{E} \leq C_{\mathcal{F}}(X)$; but it takes some effort to show that such a largest subgroup exists.

The local theory of groups proceeds via an analysis of its local subgroups. A local subsystem of a saturated fusion system $\mathcal{F}$ is the normalizer $N_{\mathcal{F}}(U)$ of a nontrivial fully normalized subgroup $U$ of $S$. We need $U$ fully normalized in order to appeal 
to Puig's Theorem 1.11 to conclude that $N_{\mathcal{F}}(U)$ is saturated; this is necessary to ensure that $N_{\mathcal{F}}(U)$ has all the nice properties shared by saturated systems but not ordinary fusion systems.

We write $\mathcal{F}^{f}$ for the set of subgroups of $S$ that are fully normalized in $\mathcal{F}$. Recall the definition of radical and centric subgroups from Definition 1.13, and write $\mathcal{F}^{\text {frc }}$ for the set of fully normalized radical centric subgroups of $\mathcal{F}$.

2.2. Components and the generalized Fitting subsystem. Recall some group theoretical facts from ALSS or A2. The components of a finite group $G$ are its subnormal quasisimple subgroups. (Subnormality is the transitive extension of the normality relation.) The subgroup $E(G)$ is the central product of the components of $G$, which commute element-wise. Then the generalized Fitting subgroup of $G$ is

$$
F^{*}(G)=F(G) E(G),
$$

where $F(G)$ is the largest normal nilpotent subgroup of $G$. Recall that

$$
F(G)=\prod_{p} O_{p}(G)
$$

We have

$$
C_{G}\left(F^{*}(G)\right)=Z\left(F^{*}(G)\right) .
$$

In the classification of the finite simple groups, the prime 2 plays a special role. In particular the finite simple groups are classified in terms of their 2-local structure. Define a group $G$ to be of characteristic 2-type if for each 2-local subgroup $H$ of $G, F^{*}(H)=O_{2}(H)$. Then $G$ is of component type if for some involution $t$ in $G$, $C_{G}(t) / O\left(C_{G}(t)\right)$ has a component, where $O(G)$ is the largest normal subgroup of $G$ of odd order. Call $O(G)$ the core of $G$; in finite group theory, the cores of 2-locals are obstructions to many arguments, and as a result cause serious problems in the proof of the classification theorem. One reason why fusion systems can be easier to work with is that such "core obstructions" are not present.

In any event we have the following partition of the simple groups via their 2-local structure:

Theorem 2.4 (Gorenstein-Walter Dichotomy Theorem). Let $G$ be a finite simple group of 2-rank at least 3. Then $G$ is either of characteristic 2-type or of component type.

Recall the 2-rank $m_{2}(G)$ of a finite group $G$ is the maximum dimension of subgroup of $G$ which is the direct product of groups of order 2, regarded as a vector space over the field of order 2 .

A proof of the Dichotomy Theorem can be found in ALSS. The simple groups of 2 -rank at most 2 are "small groups" whose treatment requires special techniques. Then the two classes of generic simple groups are treated via different methods. This approach to classifying simple groups suggests a possible model for classifying simple 2-fusion systems. So we wish to translate some of the group theoretic notions above into analogous notions in the category of saturated fusion systems.

Let $\mathcal{F}$ be a saturated fusion system on a $p$-group $S$. We already have a relation of normality for fusion systems; this allows us to define the subnormality relation as the transitive extension of normality.

Define $\mathcal{F}$ to be quasisimple if $\mathcal{F}=O^{p}(\mathcal{F})$ and $\mathcal{F} / Z(\mathcal{F})$ is simple. The components of $\mathcal{F}$ are its subnormal quasisimple subsystems. Define $E(\mathcal{F})$ to be the normal 
closure in $\mathcal{F}$ of the product of the Sylow groups of the components of $\mathcal{F}$. From Theorem 6 in [A4]:

Theorem 2.5. (1) $E(G) \unlhd \mathcal{F}$.

(2) $E(G)$ is a central product of the components of $\mathcal{F}$.

(3) $O_{p}(\mathcal{F})$ centralizes $E(G)$, so the central product $F^{*}(\mathcal{F})=O_{p}(\mathcal{F}) E(\mathcal{F})$ is normal in $\mathcal{F}$.

(4) $C_{\mathcal{F}}\left(F^{*}(\mathcal{F})\right)=Z\left(F^{*}(\mathcal{F})\right)$.

We call $F^{*}(\mathcal{F})$ the generalized Fitting subsystem of $\mathcal{F}$.

Theorem 2.6. The following are equivalent:

(1) $\mathcal{F}$ is constrained.

(2) $E(\mathcal{F})=1$.

(3) $F^{*}(\mathcal{F})=O_{p}(\mathcal{F})$.

Proof. Recall $\mathcal{F}$ is constrained if for some subgroup $Q$ of $S$, we have $Q$ normal in $\mathcal{F}$ and $C_{\mathcal{F}}(Q) \leq Q$. If such a $Q$ exists, then $Q \leq O_{p}(\mathcal{F})$, so by Theorem 2.5 , $E(\mathcal{F}) \leq C_{\mathcal{F}}(Q) \leq Q$, so that (1) implies (2). By definition of $F^{*}(\mathcal{F}),(2)$ implies (3). Finally, if (3) holds, then $Q=O_{p}(\mathcal{F}) \unlhd \mathcal{F}$, and $C_{\mathcal{F}}(Q) \leq Q$ by part (4) of Theorem 2.5, so that (3) implies (1).

The following result is II.9.7 in $\mathrm{AKO}$ :

Theorem 2.7 (E-Balance Theorem). For $U \in \mathcal{F}^{f}, E\left(N_{\mathcal{F}}(U)\right) \leq E(\mathcal{F})$.

Definition 2.8. Define $\mathcal{F}$ to be of characteristic p-type if for each $1 \neq U \in \mathcal{F}^{f}$, we have $N_{\mathcal{F}}(U)$ constrained. Define $\mathcal{F}$ to be of component type if for some $U \in \mathcal{F}^{f}$ of order $p, N_{\mathcal{F}}(U)$ has a component.

Theorem 2.9 (Dichotomy Theorem for fusion systems). A saturated fusion system $\mathcal{F}$ is either of characteristic p-type or of component type.

Proof. Assume $\mathcal{F}$ is not of characteristic $p$-type. Then for some $1 \neq U \in \mathcal{F}^{f}$, $\mathcal{N}=N_{\mathcal{F}}(U)$ is not constrained. Then by Theorem 2.6 $E(\mathcal{N}) \neq 1$. Let $X$ of order $p$ in $U$ be normal in $N_{S}(U)$. There is $\alpha \in \operatorname{hom}_{\mathcal{F}}\left(N_{S}(X), S\right)$ with $\alpha(X) \in \mathcal{F}^{f}, \alpha(U)$ fully normalized in $\mathcal{M}=N_{\mathcal{F}}(\alpha(X))$, and $\alpha\left(N_{\mathcal{N}}(X)\right)=N_{\mathcal{M}}(\alpha(U))$. Then $1 \neq$ $\alpha(E(\mathcal{N}))=E\left(N_{\mathcal{M}}(\alpha(U))\right)$. By $E$-balance, $E\left(N_{\mathcal{M}}(\alpha(U))\right) \leq E(\mathcal{M})$, so $E(\mathcal{M}) \neq 1$ and hence $\mathcal{M}$ has a component. That is $\mathcal{F}$ is of component type.

Compare Theorem 2.4 (the Dichotomy Theorem for groups) with Theorem 2.9 (the Dichotomy Theorem for fusion systems). The result for fusion systems is simpler in various ways. It is a result for all fusion systems, whereas the theorem for groups (as stated here) is a result for simple groups. The theorem on fusion systems requires no condition on the 2-rank to exclude "small groups". Finally (and most important) in the theorem for groups, $G$ is of component type if $C_{G}(t)$ has a component modulo its core for some involution $t$, whereas in the theorem on fusion systems the corresponding definition imposes the stronger condition that the centralizer of $t$ have a component.

Saturated 2-fusion systems seem to be better behaved than saturated $p$-fusion systems at larger primes $p$. In any event, as the classification of simple groups is primarily in terms of the 2-locals in such groups, it would appear that 2-fusion systems are of most interest in any attempt to simplify the classification of the 
simple groups using the theory of fusion systems. Thus most of our focus in the remainder of section 2 will be on 2-fusion systems.

As in the case of groups, one should probably take an inductive approach to a classification of simple 2-fusion systems, by imposing some assumptions on the simple fusion systems involved in local subsystems. For example we probably need to demand that for each fully centralized involution $t$ in $S$ and each component $\mathcal{C}$ of $C_{\mathcal{F}}(t), \mathcal{C} / Z(\mathcal{C})$ is in the class $\mathcal{K}$ of "known" simple 2 -fusion systems. We will discuss $\mathcal{K}$ in the next section.

\subsection{Known simple groups and simple fusion systems.}

Definition 2.10. Write $\mathfrak{K}$ for the class of known nonabelian finite simple groups; that is, the nonabelian groups appearing in the statement of the classification theorem. Thus $\mathfrak{K}$ consists of the alternating groups, the finite simple groups of Lie type, and the 26 sporadic simple groups. Recall that we write $\mathcal{K}$ for the class of known simple 2 -fusion systems. It will develop that $\mathcal{K}$ consists of the 2 -fusion systems of certain members of $\mathfrak{K}$ together with the "exotic Solomon systems".

Some simple fusion systems are exotic: they are realized as the $p$-fusion system of no finite group. (Recall that $\mathcal{F}$ is realized by $G$ if $\mathcal{F}=\mathcal{F}_{S}(G)$ for $S \in \operatorname{Syl}_{p}(G)$.)

Example 2.11. There is one known infinite class of exotic simple 2-fusion systems: the exotic Solomon systems $\operatorname{Sol}(q)$ for odd prime powers $q$. The Solomon systems are discussed in more detail in section 1.12 If $\mathcal{F}=\operatorname{Sol}(q)$, then $\mathcal{F}$ has one class $z^{\mathcal{F}}$ of involutions and $C_{\mathcal{F}}(z)$ is the 2-fusion system of $\operatorname{Spin}_{7}(q)$. In particular $\operatorname{Sol}(q)$ is of component type. Actually the isomorphism type of $\operatorname{Sol}(q)$ depends only on the 2-share of $q^{2}-1$, not on $q$.

For odd primes $p$ there are many exotic simple $p$-fusion systems; see for example Examples 1.28 and 1.30 .

Suppose on the other hand that $\mathcal{F}$ is a fusion system with $\mathcal{F}=\mathcal{F}_{S}(G)$ for some group $G$ and $S \in \operatorname{Syl}_{p}(G)$.

Remark 2.12. Let $O_{p^{\prime}}(G)$ be the largest normal subgroup of $G$ of order prime to $p$, and write $*$ for the natural homomorphism of $G$ onto $G^{*}=G / O_{p^{\prime}}(G)$. Then the map $s \mapsto s^{*}$ from $S$ to $S^{*}$ is an isomorphism of $\mathcal{F}_{S}(G)$ with $\mathcal{F}_{S^{*}}\left(G^{*}\right)$.

Thus, in seeking to realize $\mathcal{F}$ by a group $G$, using Remark 2.12, we may assume $O_{p^{\prime}}(G)=1$.

Suppose that $\mathcal{F}$ is simple and realized by $G$. Let $1 \neq H \unlhd G$. By Example 2.1, $\mathcal{F}_{S \cap H}(H) \unlhd \mathcal{F}$, and as $O_{p^{\prime}}(G)=1$, we have $S \cap H \neq 1$. Hence as $\mathcal{F}$ is simple, $\mathcal{F}=\mathcal{F}_{S \cap H}(H)$. This shows:

Remark 2.13. If $\mathcal{F}$ is simple and realized by a finite group, then $\mathcal{F}$ is realized by a finite simple group.

But what about the converse? In II.9.8.d in $\mathrm{AKO}$, there is a pair of conditions that are necessary and sufficient for $\mathcal{F}$ to be simple. Using these conditions, it is shown in II.13.1 of [AKO that:

Lemma 2.14. Assume $\mathcal{F}=\mathcal{F}_{S}(G)$ satisfies

(1) there exists no nontrivial proper subgroup of $S$ strongly closed in $S$ with respect to $G$, and

(2) $\operatorname{Aut}_{G}(S)=\left\langle\operatorname{Aut}_{O^{p^{\prime}}\left(N_{G}(R)\right)}(S): R \in \mathcal{F}^{\text {frc }}\right\rangle$.

Then $\mathcal{F}$ is simple. 
So the first step in deciding whether the $p$-fusion system $\mathcal{F}$ of some $G \in \mathfrak{K}$ is simple is to check for which of those groups $G$ there exists a nontrivial proper strongly closed subgroup of $S$. This is accomplished when $p=2$ by Foote in [Fo] and for odd $p$ by Foote and Flores in [FF]. We will discuss only the case $p=2$.

Define a simple group $G$ with Sylow 2-subgroup $S$ to be a Goldschmidt group if either $G$ is of Lie type of Lie rank 1 and even characteristic or if $S$ is abelian. These turn out to be the simple groups such that $S$ possesses a nontrivial abelian subgroup strongly closed in $S$ with respect to $G$; they were classified by Goldschmidt in $[\mathrm{Gd} 2$. By $\mathrm{Fo}$, condition (1) of Lemma 2.14 fails if and only if $G$ is Goldschmidt; moreover, in that case, $S \unlhd \mathcal{F}$, so $\mathcal{F}$ is not simple. On the other hand when $G$ is not Goldschmidt, it can be checked (with some difficulty) that condition (2) of Lemma 2.14 is satisfied. For example many of the necessary checks appear in Chapter 16 of [A4. Hence:

Theorem 2.15. If $G \in \mathfrak{K}$, then the 2 -fusion system of $G$ is simple if and only if $G$ is not Goldschmidt.

Corollary 2.16. $\mathcal{K}$ consists of exotic Solomon systems together with the 2-fusion systems of those members of $\mathfrak{K}$ that are not Goldschmidt.

The situation for odd primes is more complicated. Using [FF], one can determine the $p$-Goldschmidt groups: those $K \in \mathfrak{K}$ such that condition (1) of Lemma 2.14 is not satisfied. However, even if $G$ is not $p$-Goldschmidt, its $p$-fusion system need not be simple from the criterion in II.9.8.d in [AKO].

2.4. Reduced fusion systems and small extensions of simple systems. Having determined $\mathcal{K}$, it is still important to go on to determine certain extensions $\mathcal{F}$ of simple systems $\mathcal{D}$ in $\mathcal{K}$. There are many reasons to be interested in such extensions, but most immediately, such knowledge is important in attempting to classify simple 2 -fusion systems.

Definition 2.17. For example, define a saturated $p$-fusion system $\mathcal{F}$ to be almost simple if $F^{*}(\mathcal{F})$ is simple and not the fusion system of the group of order $p$.

At the very least we want to determine the almost simple 2 -fusion systems with $F^{*}(\mathcal{F})$ in $\mathcal{K}$ and the quasisimple systems with $\mathcal{F} / Z(\mathcal{F})$ in $\mathcal{K}$. Determining such extensions involves the notion of a tame system, discussed in section 3.3 .

Definition 2.18. Let $\mathcal{F}$ be a saturated fusion system on a finite $p$-group $S$. Proceeding as in Definition II.12.2 in [AKO, which in turn follows Puig in Chapter 19 of [Pg5], set $S_{0}=S, \mathcal{F}_{0}=\mathcal{F}$, and proceeding recursively, for $m>0$ define $S_{2 m+1}=$ $S_{2 m}, \mathcal{F}_{2 m+1}=O^{p^{\prime}}\left(\mathcal{F}_{2 m}\right), S_{2 m+2}=\mathfrak{h} \mathfrak{n p}\left(\mathcal{F}_{2 m+1}\right)$, and $\mathcal{F}_{2 m+2}=O^{p}\left(\mathcal{F}_{2 m+1}\right)$. Call the series $\mathcal{F}=\mathcal{F}_{0} \geq \mathcal{F}_{1} \geq \cdots$ the Puig series for $\mathcal{F}$ and write $\mathcal{F}^{\infty}$ for the last term in the Puig series. Each member $\mathcal{E}$ of the Puig series is a characteristic subsystem of $\mathcal{F}$; that is $\mathcal{E} \unlhd \mathcal{F}$ and $\mathcal{E}$ is $\operatorname{Aut}(\mathcal{F})$-invariant, in that for each $\alpha \in \operatorname{Aut}(\mathcal{F})$ we have $\alpha(\mathcal{E})=\mathcal{E}$. We say that $\mathcal{F}$ is Puig solvable if $\mathcal{F}^{\infty}=1$. We will encounter Puig solvable systems in section 2.8

Definition 2.19. The reduction of $\mathcal{F}$ is defined to be $\left(C_{\mathcal{F}}\left(O_{p}(\mathcal{F})\right) / Z\left(O_{p}(\mathcal{F})\right)\right)^{\infty}$. We write $\mathfrak{r e d}(\mathcal{F})$ for the reduction of $\mathcal{F}$. Define $\mathcal{F}$ to be reduced if $O_{p}(\mathcal{F})=1$ and $O^{p}(\mathcal{F})=\mathcal{F}=O^{p^{\prime}}(\mathcal{F})$. By Proposition 2.2 in AOV1, the reduction of $\mathcal{F}$ is reduced. 
In Definition 3.3 we find the definition of a tame fusion system. By Theorem A in AOV1]:

Theorem 2.20. If $\mathfrak{r e d}(\mathcal{F})$ is tame, then $\mathcal{F}$ is also tame, so in particular $\mathcal{F}$ is realizable.

Definition 2.21. Define $\mathcal{F}$ to be a small extension of a simple system $\mathcal{D}$ if $E(\mathcal{F})$ is quasisimple and $E(\mathcal{F}) / Z(E(\mathcal{F})) \cong \mathcal{D}$.

Lemma 2.22. Assume $\mathcal{F}$ is a small extension of a simple system $\mathcal{D}$, and let $Q=$ $O_{p}(\mathcal{F})$ and $\mathcal{X}=C_{\mathcal{F}}(Q)$. Then

(1) $F^{*}(\mathcal{X})=Z(Q) E(\mathcal{F})$.

(2) $\mathcal{F}_{0}=\mathcal{X} / Z(Q)$ is almost simple with $F^{*}\left(\mathcal{F}_{0}\right) \cong \mathcal{D}$.

(3) Assume $\mathcal{D}$ is tamely realized by some simple group $K$. Then $\mathcal{F}_{0}^{\infty}=F^{*}\left(\mathcal{F}_{0}\right)$ and $\mathcal{F}$ is realized by a group $G$ with $F^{*}(G)=Q E(G)$, with $E(G)$ quasisimple with $p$-fusion system $E(\mathcal{F})$, and with $E(G) / Z(E(G)) \cong K$.

Proof. Set $\mathcal{E}=E(\mathcal{F})$. From 2.5, $F^{*}(\mathcal{X})=O_{p}(\mathcal{X}) E(\mathcal{X})$ is characteristic in $\mathcal{X}$ and hence normal in $\mathcal{F}$. By 10.3 in [A4], $E(\mathcal{X})=\mathcal{E}$. Further $O_{p}(\mathcal{X}) \leq \mathcal{X}=C_{\mathcal{F}}(Q)$, so $O_{p}(\mathcal{X})=Z(Q)$, completing the proof of $(1)$.

As $Z(\mathcal{E}) \leq Z(Q)$, the image $\mathcal{E}_{0}$ of $\mathcal{E}$ in $\mathcal{F}_{0}$ is simple. By 2.21, $\mathcal{E}_{0} \cong \mathcal{D}$. As $\mathcal{E} \unlhd \mathcal{F}$, we have $\mathcal{E}_{0} \unlhd \mathcal{F}_{0}(\operatorname{cf} 8.9$ in A3] $)$. The preimage of $O_{p}\left(\mathcal{F}_{0}\right)$ in $\mathcal{F}_{0}$ is contained in $O_{p}(\mathcal{X})=Z(Q)$ by $(1)$, so $O_{p}\left(\mathcal{F}_{0}\right)=1$. Let $\mathcal{E}_{1}$ be the product of the components of $\mathcal{F}_{0}$ distinct from $\mathcal{E}_{0}$. By 8.10 in [A3], the preimage $\mathcal{E}_{2}$ of $\mathcal{E}_{1}$ in $\mathcal{X}$ is normal in $\mathcal{X}$. Then by 7.15 in [A4], if $\mathcal{E}_{1} \neq 1$, then $O^{p}\left(\mathcal{E}_{2}\right)$ is the product of components of $\mathcal{F}$, contradicting $\mathcal{E}=E(\mathcal{F})$. Therefore $F^{*}\left(\mathcal{F}_{0}\right)=\mathcal{E}_{0}$, so (2) holds.

Assume the setup in $(3)$. By $(2), \mathcal{E}_{0}=F^{*}\left(\mathcal{F}_{0}\right) \cong \mathcal{D}$, and by the hypothesis of (3), $\mathcal{D}$ is tamely realized by the simple group $K$. Therefore by Theorem 2.3 in [07], $\mathcal{E}_{0}=\mathcal{F}_{0}^{\infty}$. Then $\mathcal{E}_{0}=\mathfrak{r} \mathfrak{d}(\mathcal{F})$ is tame, so by $2.20, \mathcal{F}$ is tamely realized by some group $G$. Indeed from the proof of 2.20 in [AOV1, Theorem 2.20], $\mathcal{F}^{*}=Q * \mathcal{X}$ is tamely realized by a group $G^{*} \unlhd G$ with $F^{*}\left(G^{*}\right)=Q E(G)$ and $\mathcal{E}$ the $p$-fusion system of $E(G)$. As $\mathcal{X} \leq \mathcal{F}^{*}$, we have $C_{G}(Q) \leq G^{*}$, so $C_{G}(Q E(G)) \leq C_{G^{*}}(Q E(G))=Z(Q)$, and hence $F^{*}\left(G^{*}\right)=Q E(G)$, completing the proof of $(3)$.

Remark 2.23. We would like to know the small extensions $\mathcal{F}$ of simple systems $\mathcal{D}$ such that $\mathcal{D}$ is tamely realized by some simple group $K \in \mathfrak{K}$. From Lemma 2.22(3) we know that $\mathcal{F}$ is tamely realized by some group $G$ with $F^{*}(G)=O_{p}(G) E(G)$ and $E(G)$ quasisimple with $p$-fusion system $E(\mathcal{F})$ and $E(G) / Z(E(G)) \cong K$. Hence we can make use of group extension theory to pin down $\mathcal{F}$.

For example if $\mathcal{F}$ is quasisimple, then $\mathcal{F}_{0}=\mathcal{F} / Z(\mathcal{F}) \cong \mathcal{D}$ is simple. Hence the quasisimple systems $\mathcal{F}$ with simple factor system $\mathcal{D}$ are precisely the $p$-fusion systems of the various coverings of $K$, and those coverings are known.

Or suppose that $\mathcal{F}$ is almost simple. Then by Lemma $2.22(3), \mathcal{F}$ is tamely realized by an almost simple group $G$ with $F^{*}(G)=K$, and again all such almost simple groups are known.

2.5. Transfer in fusion systems. An important tool in local group theory is the notion of a transfer homomorphism. It takes as its starting point the transfer in group homology and cohomology; for example, the transfer homomorphism from the cohomology of $S$ to that of $G$, when $G$ is a finite group and $S \in \operatorname{Syl}_{p}(G)$. It can be used as a tool for converting local data, such as fusion within a Sylow $p$-subgroup 
of $G$, to global data such as showing that $O^{p}(G)$ is a proper subgroup of $G$. Thus transfer can be used to produce normal subgroups of a finite group $G$.

Example 2.24. Perhaps the transfer theorem most used in local group theory is Thompson transfer. Let $S \in \operatorname{Syl}_{2}(G)$, and let $T$ be a subgroup of index 2 in $S$. Suppose $t$ is an involution in $S$ such that each $G$-conjugate of $t$ contained in $S$ is in $S-T$. Then $t \notin O^{2}(G)$. The proof of Thompson transfer is elementary.

The construction and properties of the $H_{1}$-transfer homomorphism for fusion systems were described in AKO, $\S$ I.8]. This transfer is an injective homomorphism from $H_{1}(\mathcal{F} ; \mathbb{Z}) \cong S / \mathfrak{f o c}(\mathcal{F})$ to $H_{1}(S ; \mathbb{Z}) \cong S /[S, S]$ (see [AKO, Proposition I.8.4]). It gives, for example, a tool for showing that certain elements in $S$ cannot lie in $\mathfrak{f} \mathfrak{c}(\mathcal{F})$, and hence (via Theorem $1.23(\mathrm{a})$ ) a tool for showing under certain conditions that $O^{p}(\mathcal{F})<\mathcal{F}$. For some other applications of the transfer, we refer to [Cr2, $\left.\S 7.7\right]$ and DGMP2].

The following is a special case of a transfer theorem due to Lynd in Lyn1. This special case is essentially a Thompson transfer for saturated fusion systems.

Theorem 2.25 (Lynd transfer). Assume $\mathcal{F}$ is a saturated fusion system on a 2 group $S, T$ is a subgroup of $S$ of index 2, and $i$ is an involution in $S$ such that $i^{\mathcal{F}} \cap \mathcal{F}^{f} \subseteq S-T$. Then $i \notin O^{2}(\mathcal{F})$.

2.6. Classifying simple 2-fusion systems: The odd-even partition. We now turn to the problem of classifying simple 2 -fusion systems. So assume $\mathcal{F}$ is a saturated fusion system on a finite 2-group $S$.

One possible approach to the problem is to divide the analysis into the two cases arising in the Dichotomy Theorem. However for technical reasons, it seems likely that it is better to move the dividing line between the two halves of the partition, so as to determine only a large subclass of the class of systems of component type, and then classify the remaining systems, which properly include the systems of characteristic 2-type. We begin by describing the resulting partition of 2-fusion systems. But first we need a basic definition and lemma.

Definition 2.26. For $P \subseteq S$, define $\mathfrak{A}(P)=\left\{\phi \in \operatorname{Hom}_{\mathcal{F}}\left(N_{S}(P), S\right): \phi(\langle P\rangle) \in \mathcal{F}^{f}\right\}$.

Lemma 2.27. For each $P \leq S$ and $Q \in \mathcal{F}^{f} \cap P^{\mathcal{F}}$ there exists $\alpha \in \mathfrak{A}(P)$ with $\alpha(P)=Q$.

Proof. See I.2.6 in AKO.

Recall the local subsystems of $\mathcal{F}$ are those of the form $N_{\mathcal{F}}(U)$ for $1 \neq U \in \mathcal{F}^{f}$. Lemma 2.27 says there are enough local subsystems of $\mathcal{F}$.

Definition 2.28. Given a quasisimple subsystem $\mathcal{C}$ of $\mathcal{F}$, define $\mathcal{I}(\mathcal{C})$ to be the set of involutions $t \in S$ centralizing $\mathcal{C}$ such that for $\alpha \in \mathfrak{A}(t), \alpha(\mathcal{C})$ is a component of $C_{\mathcal{F}}(\alpha(t))$. Define $\mathfrak{C}(\mathcal{F})$ to consist of those quasisimple subsystems $\mathcal{C}$ of $\mathcal{F}$ such that $\mathcal{I}(\mathcal{C}) \neq \varnothing$. In particular $\mathcal{F}$ is of component type if and only if $\mathfrak{C}(\mathcal{F}) \neq \varnothing$. We call $\mathfrak{C}(\mathcal{F})$ the set of components of involution centralizers of $\mathcal{F}$.

Since our attempts at classification proceed by induction on the "order" of $\mathcal{F}$, we will assume that for each $\mathcal{C} \in \mathfrak{C}(\mathcal{F})$, we have $\mathcal{C} / Z(\mathcal{C}) \in \mathcal{K}$ the class of known simple 2-fusion systems. We then apply:

Theorem 2.29. For each $\mathcal{L} \in \mathcal{K}$, either $\mathcal{L}$ is an exotic Solomon system or $\mathcal{L}$ is tamely realized by some $K(\mathcal{L}) \in \mathfrak{K}$. 
Theorem 2.29 allows us to appeal to the extension theory in section 2.4. For example, together with Remark 2.23, it says that each $\mathcal{C} \in \mathfrak{C}(\mathcal{F})$ is a known quasisimple system. The theorem follows from Theorems 3.5 and 3.6 in the next section.

Definition 2.30. Define a quasisimple system $\mathcal{F}$ to be intrinsic if $Z(\mathcal{F}) \neq 1$. Define $\mathcal{F}$ to be subintrinsic if either $\mathcal{F}$ is intrinsic or $\mathfrak{C}(\mathcal{F})$ contains an intrinsic member.

Definition 2.31. Define $\mathcal{J}(S)$ to consist of the involutions $j \in S$ such that $m_{2}(S)=m_{2}\left(C_{S}(j)\right)$. For $\mathcal{C} \in \mathfrak{C}(\mathcal{F})$ set $\mathcal{J}(\mathcal{C})=\mathcal{J}(S) \cap \mathcal{I}(\mathcal{C})$. Finally set $\mathfrak{C}_{J}(\mathcal{F})=$ $\{\mathcal{C} \in \mathfrak{C}(\mathcal{F}): \mathcal{J}(\mathcal{C}) \neq \varnothing\}$.

Definition 2.32. Define $\mathcal{F}$ to be an odd system if either

(1) $\mathfrak{C}(\mathcal{F})$ contains a subintrinsic member, or

(2) $\mathfrak{C}(\mathcal{F})$ contains no subintrinsic members and $\mathfrak{C}_{J}(\mathcal{F}) \neq \varnothing$.

Define $\mathcal{F}$ to be an even system if $\mathcal{F}$ is not odd. This gives us our partition of the class of saturated 2-fusion systems into odd and even systems.

The exotic Solomon systems are odd. The 2-fusion systems of almost all groups of Lie type in odd characteristic are odd; the systems of $L_{2}(q), q$ odd, are exceptions. The 2-fusion systems of groups of Lie type and characteristic 2 are of characteristic 2 -type, and hence even. The 2-fusion systems of most alternating groups are odd. Some of the systems of sporadic groups are odd and some are even.

The definition of the class of odd systems is chosen so as to avoid the necessity of characterizing certain 2-fusion systems of component type from the point of view of a small involution centralizer with a component. Thus the class of odd systems should be easier to treat than the larger class of systems of component type. On the other hand the class of even systems properly contains the systems of characteristic 2-type. Recall from Definition 2.8 that if $\mathcal{F}$ is of characteristic 2-type, then each local subsystem of $\mathcal{F}$ is constrained, and hence possesses a model. When $\mathcal{F}$ is even it satisfies the following weaker property:

Lemma 2.33. Assume that $\mathcal{F}$ is even. Then for each $1 \neq U \in \mathcal{F}^{f}$ with $m_{2}\left(N_{S}(U)\right)=$ $m_{2}(S), N_{\mathcal{F}}(U)$ is constrained and hence possesses a model.

Proof. If $\mathcal{N}=N_{\mathcal{F}}(U)$ is constrained, then by the Model Theorem 1.24, $\mathcal{N}$ has a model.

Suppose on the other hand that $\mathcal{N}$ is not constrained. Then by Theorem 2.6 , $E(\mathcal{N}) \neq 1$. Let $T$ be Sylow in $\mathcal{N}$; by assumption, $m_{2}(T)=m_{2}(S)$. Let $u$ be an involution in $U \cap Z(T), \alpha \in \mathfrak{A}(u), j=\alpha(u)$, and $\mathcal{E}=C_{\mathcal{F}}(j)$. Then $u \in \mathcal{N}^{f}$, so $\alpha(U) \in \mathcal{E}^{f}$. Also $E(\mathcal{N})=E\left(C_{\mathcal{N}}(u)\right)$ by 10.3 in [A4]. Therefore $\alpha(E(\mathcal{N}))=$ $E\left(N_{\mathcal{E}}(\alpha(U))\right.$, so by the $E$-Balance Theorem 2.7, $\alpha(E(\mathcal{N})) \leq E(\mathcal{E})$. Therefore there is a component $\mathcal{C}$ of $\mathcal{E}$ and $j \in \mathcal{J}(\mathcal{C})$ with $m_{2}\left(C_{S}(j)\right)=m_{2}(T)=m_{2}(S)$, so $\mathfrak{C}_{J}(\mathcal{F}) \neq \varnothing$, contradicting $\mathcal{F}$ even.

2.7. Odd 2-fusion systems and groups. In this subsection we assume the following hypothesis:

Hypothesis 2.34. $\mathcal{F}$ is a saturated fusion system on a finite 2-group $S$ such that for each $\mathcal{C} \in \mathfrak{C}(\mathcal{F}), \mathcal{C} / Z(\mathcal{C}) \in \mathcal{K}$ and, if $\mathcal{C}$ is not an exotic Solomon system, then $\mathcal{C} / Z(\mathcal{C})$ is tamely realized by some $K(\mathcal{C} / Z(\mathcal{C})) \in \mathfrak{K}$. 
Our objective is to show that if $\mathcal{F}$ is simple, then $\mathcal{F} \in \mathcal{K}$. In this subsection we consider the case where $\mathcal{F}$ is odd. We give a very sketchy outline of a program aimed at classifying the odd systems $\mathcal{F}$ satisfying Hypothesis 2.34 , and then give some indication of how such a classification might be used to classify the corresponding class of "odd" finite simple groups. Finally we discuss some properties of "even" simple groups.

The expository article A8 gives a fairly detailed discussion of a program of the first author aimed at classifying the simple odd 2-fusion systems satisfying Hypothesis 2.34, Roughly speaking, the program proceeds as follows.

There is an "ordering" on $\mathfrak{C}(\mathcal{F})$. One begins by showing that if $\mathcal{C} \in \mathfrak{C}(\mathcal{F})$ is "maximal" in this ordering or if Definition 2.32 (2) holds and $\mathcal{C}$ is "maximal" in $\mathfrak{C}_{J}(\mathcal{F})$, then $\mathcal{C}$ is terminal: again roughly, $\mathcal{I}(\mathcal{C})$ includes most involutions centralizing $\mathcal{C}$. In many situations this makes it possible to prove $\mathcal{C}$ is standard; that is, one can define the "centralizer" $\mathcal{Q}$ of $\mathcal{C}$ in $\mathcal{F}$ and show that $\mathcal{Q}$ is tightly embedded in $\mathcal{F}$. This in turn shows that a Sylow group $Q$ of $\mathcal{Q}$ is elementary abelian or cyclic and $N_{\mathcal{F}}(Q)$ is a small extension of $\mathcal{C} / Z(\mathcal{C})$. By Hypothesis 2.34, $\mathcal{C} / Z(\mathcal{C})$ is tamely realized by some simple group $K$, so from Lemma $2.22(3)$, we know $N_{\mathcal{F}}(Q)$ is realized by some fairly well known group. Then we need to solve the corresponding standard form problem for $\mathcal{C}$ : show that $\mathcal{F}$ is determined up to isomorphism by $N_{\mathcal{F}}(Q)$.

Almost all the 2-fusion systems of groups of Lie type in odd characteristic emerge in the case of Definition 2.32(1), along with systems of some of the sporadic groups and the exotic Solomon systems. The systems of the alternating groups and some of the remaining sporadics emerge in the case of Definition 2.32(2). Fortunately, it is not necessary to solve the standard form problems for the systems of most groups of Lie type and odd characteristic, as there is a characterization of such systems similar to the characterization of their groups in [A1.

We also want to study the corresponding class of odd groups. Given a finite group $G$, define $\mathfrak{C}(G), \mathfrak{C}_{J}(G)$ to be the set of components of the groups $C_{G}(t) / O\left(C_{G}(t)\right)$ as $t$ ranges over the set $\mathcal{I}(G)$ of involutions of $G$, the set $\mathcal{J}(G)$ of those $j \in \mathcal{I}(G)$ with $m_{2}\left(C_{G}(t)\right)=m_{2}(G)$, respectively. Define intrinsic and subintrinsic members of $\mathfrak{C}(G)$ by analogy with the corresponding definitions for fusion systems in Definition 2.30. We begin with groups satisfying:

Hypothesis 2.35. $G$ is a finite group such that for each $L \in \mathfrak{C}(G)$, we have $L / Z(L) \in \mathfrak{K}$.

Definition 2.36. Define a group $G$ to be odd if either

(1) $\mathfrak{C}(G)$ contains a subintrinsic member, or

(2) $\mathfrak{C}(G)$ contains no subintrinsic members that are not Goldschmidt and $\mathfrak{C}_{J}(G)$ contains a member that is not Goldschmidt.

Modulo some complications introduced by possible members of $\mathfrak{C}(G)$ which are coverings of Goldschmidt groups, the odd groups are the groups whose 2-fusion systems are odd.

We want to use a classification of the simple odd 2-fusion systems satisfying Hypothesis 2.34 to classify the odd finite simple groups satisfying Hypothesis 2.35. The following result suggests that this might be possible.

Theorem 2.37. Assume that $G$ is an almost simple group satisfying Hypothesis 2.35 and $F^{*}(G)$ is not a Goldschmidt group. Assume the 2-fusion system of each 
member of $\mathfrak{K}$ is tamely realized by some member of $\mathfrak{K}$. Then the 2-fusion system $\mathcal{F}$ of $G$ is almost simple, satisfies Hypothesis 2.34, and if $G=O^{2}(G)$, then also $\mathcal{F}=O^{2}(\mathcal{F})$.

Define a group $G$ to be even if $G$ is not odd. Assuming that the odd groups can be classified using this approach, we are left with the problem of classifying the even finite simple groups. It can be shown that:

Theorem 2.38. Assume that $G$ is an almost simple even group with $m_{2}(G) \geq 3$ satisfying Hypothesis 2.35 . Then either

(1) for each $j \in \mathcal{J}(G), O\left(C_{G}(j)\right)=1$, or

(2) $m_{2}(G)=3$ and either $G \cong S_{7}$ or $F^{*}(G) \cong L_{2}\left(q^{2}\right)$ for some $q \equiv \pm 3 \bmod 8$.

Theorem 2.39. Assume that $G$ is an almost simple even group satisfying Hypothesis 2.35 and some member of $\mathfrak{C}_{J}(G)$ is isomorphic to $L_{2}(q), q \equiv \pm 3 \bmod 8, U_{3}(4)$, ${ }^{2} G_{2}(q)$, or $J_{1}$. Then $F^{*}(G) \in \mathfrak{K}$.

Corollary 2.40. Assume that $G$ is a simple even group with $m_{2}(G) \geq 3$ satisfying Hypothesis 2.35. Then $O\left(C_{G}(t)\right)=1$ for each involution $t$ in $G$.

In summary, if $G$ is an even simple group minimal subject to not being in $\mathfrak{K}$, then $G$ closely resembles what is termed a group of even type in Definition 21.3 in GLS1. Thus one could hope to use the work of Gorenstein, Lyons, and Solomon on groups of even type to determine the even simple groups in the event one cannot determine the simple even 2 -fusion systems.

2.8. Even 2-fusion systems. The notion of an even 2-fusion system is of fairly recent vintage, so little work has been done on such systems. There are however some results on 2-fusion systems of characteristic 2-type. We mention one such result that is a fusion system analogue of the classic theorem of John Thompson on $N$-groups. Recall an $N$-group is a finite simple group in which all local subgroups are solvable. In [T], Thompson classifies the $N$-groups. This work served as a model for the classification of the finite simple groups, particularly the groups of characteristic 2 -type. The $N$-group theorem was later extended by various authors to a classification of the $N_{2}$-groups, where an $N_{2}$-group is a finite group in which all 2-local subgroups are solvable.

Definition 2.41. An $N$-system is a saturated 2-fusion system in which all local subsystems are Puig solvable.

Recall the definition of Puig solvability in Definition 2.18, $\mathcal{F}$ is Puig solvable if $\mathcal{F}^{\infty}=1$, where $\mathcal{F}^{\infty}$ is the last term in the Puig series for $\mathcal{F}$. The Puig series of a fusion system is analogous to the derived series of a finite group $G$, and $G$ is solvable if and only if its derived series descends to 1 . Thus Puig solvability is analogous to solvability in groups and $N$-systems are analogous to $N_{2}$-groups.

It can be shown (cf. II.12.4 in $\mathrm{AKO}$ ) that $\mathcal{F}$ is Puig solvable if and only if $\mathcal{F}$ is constrained and the model for $\mathcal{F}$ is solvable. Hence each $N$-system is of characteristic 2 -type and the 2-fusion system of each $N_{2}$-group is an $N$-system. In A9 the $N$-systems are classified and this theorem is used to give a new simplified proof of the theorem classifying the $N_{2}$-groups. 
2.9. Sectional 2 -rank $\leq 4$. Finally we come to an alternate approach to the study of simple 2-fusion systems. We begin with a few definitions.

Let $G$ be a finite group. A section of $G$ is group of the form $H / K$ where $K \unlhd$ $H \leq G$. The sectional 2-rank of $G$ is the maximum 2-rank of a section of $G$.

In 1974 in GH], Gorenstein and Harada classified the finite simple groups of sectional 2-rank at most 4 . At the time, the sectional 2-rank 4 theorem served as part of the proof of the Gorenstein-Walter Dichotomy Theorem 2.4 but later Harada made an observation which made it possible to avoid the need to quote the sectional 2-rank theorem in proving the Dichotomy Theorem. Still the result remained important as a means for identifying small simple groups in many situations.

In O6, Oliver proves an analogue of the Gorenstein-Harada theorem for 2-fusion systems. Let $\mathcal{F}$ be a saturated fusion system on a finite 2 -group $S$. Recall from Definition 2.19 that $\mathcal{F}$ is reduced if $O_{2}(\mathcal{F})=1$ and $O^{2}(\mathcal{F})=\mathcal{F}=O^{2^{\prime}}(\mathcal{F})$. Define $\mathcal{F}$ to be indecomposable if $\mathcal{F}$ is not the direct product of proper nontrivial subsystems. The main theorem of 06 says:

Theorem 2.42. Let $\mathcal{F}$ be a reduced indecomposable saturated fusion system over a finite 2 -group $S$ of sectional 2 -rank at most 4 . Then $\mathcal{F} \in \mathcal{K}$.

Actually Oliver gives an explicit list of the examples, which consists of a mixture of odd and even systems.

The proof of Theorem 2.42 focuses on the essential subgroups of $\mathcal{F}$ rather than on involution centralizers. Recall the notion of an essential subgroup is defined in Definition 1.18. Further, by the Alperin-Goldschmidt Fusion Theorem 1.19, $\mathcal{F}$ is generated by $\operatorname{Aut}_{\mathcal{F}}(S)$ and the groups $\operatorname{Aut}_{\mathcal{F}}(R)$, as $R$ ranges over the essential subgroups of $\mathcal{F}$. Therefore knowledge of its essential subgroups is enough to determine $\mathcal{F}$.

\section{Linking SYSTEMS, TRANSPORTER SYSTEMS, AND LOCALITIES}

As has been seen in earlier sections, fusion systems provide an excellent tool in many cases for manipulating the $p$-local structure of finite groups for a given prime $p$. But there are also situations where the fusion system itself does not suffice, and where one needs a more rigid structure, but one which still depends only on the $p$-local structure of a group.

As one simple example, consider the problem of constructing extensions of a fusion system $\mathcal{F}$ by a group of automorphisms of $\mathcal{F}$. Set $G=A_{6}$, choose $S \in$ $\operatorname{Syl}_{2}(G)$ (thus $S \cong D_{8}$ ), and let $\mathcal{F}=\mathcal{F}_{S}(G)$ be its fusion system. Conjugation by an element of $N_{\Sigma_{6}}(S) \backslash A_{6}$ induces an inner automorphism on $S$, and hence its class is trivial in $\operatorname{Out}(\mathcal{F})$ (as defined in $\S 1.2$ ). In other words, we cannot distinguish between an automorphism of $\mathcal{F}$ which determines the fusion system of $\Sigma_{6}$ and one which determines the fusion system of $A_{6} \times C_{2}$. However, these two fusion systems are not isomorphic, and both are split extensions of $\mathcal{F}$. So more information is needed if we want to understand such extensions on the level of fusion systems.

This is one way in which linking systems play a role. We will see that automorphisms of the linking system of $A_{6}$ (Definition 3.1) do allow us to distinguish between those induced by $\Sigma_{6}$ and the trivial ones, and also that one can directly describe extensions of linking systems by groups of automorphisms.

Linking systems also provide the "link" (hence the name) between fusion systems and homotopy theory, as will be described in section 4 
3.1. Linking systems and transporter systems of groups. We begin with some more definitions. Recall ( $\S 1.11)$ that a $p$-subgroup $P$ in a finite group $G$ is called $p$-centric in $G$ if $Z(P) \in \operatorname{Syl}_{p}\left(C_{G}(P)\right)$. This is easily seen to be equivalent to the condition that $C_{G}(P)=Z(P) \times C_{G}^{\prime}(P)$, where $C_{G}^{\prime}(P)=O^{p}\left(C_{G}(P)\right)$ has order prime to $p$.

Definition 3.1. Fix a finite group $G$ and $S \in \operatorname{Syl}_{p}(G)$.

- For $P, Q \leq S$, set $T_{G}(P, Q)=\left\{x \in G \mid{ }^{x} P \leq Q\right\}$ (the transporter set from $P$ to $Q)$.

- Let $\mathcal{T}_{S}(G)$ be the transporter system for $G$ : the category whose objects are the subgroups of $S$, and where

$$
\operatorname{Mor}_{\mathcal{T}_{S}(G)}(P, Q)=T_{G}(P, Q) .
$$

- Let $\mathcal{L}=\mathcal{L}_{S}^{c}(G)$ be the centric linking system for $G$ : the category whose objects are the $p$-centric subgroups of $G$, and where

$$
\operatorname{Mor}_{\mathcal{L}}(P, Q)=T_{G}(P, Q) / C_{G}^{\prime}(P) \text {. }
$$

- Let $\rho: \mathcal{T}_{S}(G) \longrightarrow \mathcal{F}_{S}(G)$ and $\pi: \mathcal{L}_{S}^{c}(G) \longrightarrow \mathcal{F}_{S}(G)$ be the functors which are the identity or inclusion on objects, and which send (the class of) $x \in$ $T_{G}(P, Q)$ (as a morphism from $P$ to $Q$ ) to $c_{x} \in \operatorname{Hom}_{\mathcal{F}_{S}(G)}(P, Q)$.

Such categories were first defined by Puig Pg1 as a means of more precisely formulating and studying the $p$-local properties of finite groups. In fact, the $\mathcal{F}_{S}(G)$, $\mathcal{L}_{S}^{c}(G)$, and $\mathcal{T}_{S}(G)$ are (up to equivalence of categories) just three examples of the "categories associated to $p$-localities" which he defined there.

We will see shortly (Theorem 3.8) that if $G$ and $H$ are finite groups with Sylow $p$-subgroups $S$ and $T$, respectively, then $\mathcal{F}_{S}(G) \cong \mathcal{F}_{T}(H)$ if and only if $\mathcal{L}_{S}^{c}(G) \cong$ $\mathcal{L}_{T}^{c}(H)$. This is the key reason for the importance of linking systems: $\mathcal{L}_{S}^{c}(G)$ does not contain information beyond that determined by the $p$-local structure of $G$, but it is easier in some ways to manipulate than the fusion system $\mathcal{F}_{S}(G)$.

3.2. Linking systems associated to an abstract fusion system. The properties of transporter and linking categories for groups help motivate the concept of linking and transporter systems associated to arbitrary fusion systems. Fix a saturated fusion system $\mathcal{F}$ over a $p$-group $S$, and recall that $\operatorname{Ob}(\mathcal{F})$ is the set of subgroups of $S$. Let $\mathcal{T}_{S}^{\mathcal{F}^{c}}(S) \subseteq \mathcal{T}_{S}(S)$ be the full subcategory with object set $\mathcal{F}^{c}$, the set of $\mathcal{F}$-centric subgroups of $S$.

Definition 3.2. A centric linking system associated to $\mathcal{F}$ consists of a category $\mathcal{L}$, where $\operatorname{Ob}(\mathcal{L})=\mathcal{F}^{c}$, together with a pair of functors

$$
\mathcal{T}_{S}^{\mathcal{F}^{c}}(S) \stackrel{\delta}{\longrightarrow} \mathcal{L} \stackrel{\pi}{\longrightarrow} \mathcal{F}
$$

which satisfy the following conditions.

- The functor $\delta$ is the identity on objects and injective on morphisms, while $\pi$ is the inclusion on objects and is surjective on each morphism set.

- For each $P, Q \in \mathrm{Ob}(\mathcal{L}), \delta_{P}(P) \leq \operatorname{Aut}_{\mathcal{L}}(P)$ acts freely on the set $\operatorname{Mor}_{\mathcal{L}}(P, Q)$ by precomposition and $\pi_{P, Q}$ induces a bijection from $\operatorname{Mor}_{\mathcal{L}}(P, Q) / \delta_{P}(Z(P))$ (the set of orbits under the action) onto $\operatorname{Hom}_{\mathcal{F}}(P, Q)$.

- For $P, Q \in \mathrm{Ob}(\mathcal{L})$ and $g \in T_{S}(P, Q)$, the composite functor $\pi \circ \delta$ sends $g \in \operatorname{Mor}_{\mathcal{T}}(P, Q)$ to $c_{g} \in \operatorname{Hom}_{\mathcal{F}}(P, Q)$. 
- For $P, Q \in \operatorname{Ob}(\mathcal{L}), \psi \in \operatorname{Mor}_{\mathcal{L}}(P, Q)$, and $g \in P$, the following square commutes in $\mathcal{L}$.

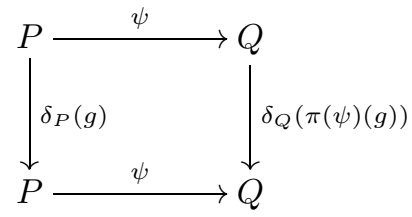

By [O3, Proposition 4(f)], all morphisms in a linking system $\mathcal{L}$ are monomorphisms and epimorphisms in the categorical sense: for any

$$
P \underset{\varphi^{\prime}}{\stackrel{\varphi}{\rightleftarrows}} Q \underset{\psi^{\prime}}{\stackrel{\psi}{\rightleftarrows}} R
$$

in $\mathcal{L}, \psi \circ \varphi=\psi \circ \varphi^{\prime}$ implies $\varphi=\varphi^{\prime}$, and $\psi \circ \varphi=\psi^{\prime} \circ \varphi$ implies $\psi=\psi^{\prime}$. This plays an important role when working with linking systems.

When $P \leq Q$ and both are objects in a linking system $\mathcal{L}$, we regard $\delta_{P, Q}(1) \in$ $\operatorname{Mor}_{\mathcal{L}}(P, Q)$ as the "inclusion" of $P$ in $Q$ in the category $\mathcal{L}$. This in turn allows us to talk about "restrictions" and "extensions" of morphisms in $\mathcal{L}$; they are unique (if they exist) since all morphisms are monomorphisms and epimorphisms.

It is not hard to show, when $G$ is a finite group and $S \in \operatorname{Syl}_{p}(G)$, that $\mathcal{L}_{S}^{c}(G)$ is a centric linking system associated to $\mathcal{F}_{S}(G)$ BLO2, $\S 1$ ].

One problem, when working with centric linking systems, is that a morphism (or even an inclusion) of fusion systems $\mathcal{E} \longrightarrow \mathcal{F}$ need not send $\mathcal{E}$-centric subgroups to $\mathcal{F}$-centric subgroups. As a consequence, there need not be a corresponding functor from a centric linking system associated to $\mathcal{E}$ to one associated to $\mathcal{F}$. In many cases, one can get around this problem by modifying the above definition to allow more general sets of objects. We refer to [AKO, Definition III.4.1] for a definition of linking systems in this more general context. Recently, Ellen Henke He5] has proposed an even more general definition of "subcentric linking systems" which promises to be useful in more general situations.

Transporter systems associated to $\mathcal{F}$ can be thought of as a further generalization of linking systems. We refer the reader to [OV1, §3] for the precise definition as well as some of the basic properties of transporter systems. However, in contrast to the situation for linking systems (see section 3.4), there can be many transporter systems associated to the same fusion system and with the same objects. As one might expect, given the name, $\mathcal{T}_{S}(G)$ (see Definition 3.1) is a transporter system associated to $\mathcal{F}_{S}(G)$. Conversely, if $\mathcal{T}$ is a transporter system associated to $\mathcal{F}$ and $\operatorname{Ob}(\mathcal{T})$ includes all subgroups of $S$, then $\mathcal{F}$ is realized by the finite group $G=\operatorname{Aut}_{\mathcal{T}}(1)$ and $\mathcal{T} \cong \mathcal{T}_{S}(G)$ OV1, Proposition 3.11].

3.3. Automorphisms, tameness, and extensions. For each object $P$ in a centric linking system $\mathcal{L}$, the group $\operatorname{Aut}_{\mathcal{L}}(P)$ contains a subgroup $\delta_{P}(P)$ isomorphic to $P$, which we refer to as the "distinguished subgroup". For various reasons, the natural automorphisms to look at for $\mathcal{L}$ turn out to be the automorphisms of $\mathcal{L}$ (as a category) which preserve distinguished subgroups and inclusions.

Definition 3.3 ([AOV1, Definition 1.13 and Lemma 1.14]). Let $\mathcal{L}$ be a centric linking system associated to a saturated fusion system $\mathcal{F}$ over the $p$-group $S$. 
- Let $\operatorname{Aut}(\mathcal{L})$ be the group of automorphisms of the category $\mathcal{L}$ which sends distinguished subgroups to distinguished subgroups and inclusions to inclusions.

- Set $\operatorname{Out}(\mathcal{L})=\operatorname{Aut}(\mathcal{L}) /\left\{c_{\gamma} \mid \gamma \in \operatorname{Aut}_{\mathcal{L}}(S)\right\}$. Here, $c_{\gamma} \in \operatorname{Aut}(\mathcal{L})$ sends an object $P$ to $\pi(\gamma)(P)$ (recall $\left.\pi(\gamma) \in \operatorname{Aut}_{\mathcal{F}}(S)\right)$, and acts on morphisms via conjugation by $\gamma$ and its restrictions.

- Let $\mu_{\mathcal{L}}: \operatorname{Out}(\mathcal{L}) \longrightarrow \operatorname{Out}(\mathcal{F})$ be the homomorphism which sends the class of $\alpha \in \operatorname{Aut}(\mathcal{L})$ to the class of $\left.\alpha_{S}\right|_{\delta_{S}(S)}$. (Here, $\alpha_{S} \in \operatorname{Aut}\left(\operatorname{Aut}_{\mathcal{L}}(S)\right)$, and this restricts to an automorphism of $\delta_{S}(S) \cong S$ since $\alpha \in \operatorname{Aut}(\mathcal{L})$.)

Definition 3.4. When $G$ is a finite group and $S \in \operatorname{Syl}_{p}(G)$, let

$$
\kappa_{G}: \operatorname{Out}(G) \cong N_{\mathrm{Aut}(G)}(S) / \operatorname{Aut}_{N_{G}(S)}(G) \longrightarrow \operatorname{Out}\left(\mathcal{L}_{S}^{c}(G)\right)
$$

be the homomorphism which sends the class of $\beta \in N_{\text {Aut }(G)}(S)$ to the class of the induced automorphism of $\mathcal{L}_{S}^{c}(G)$. The fusion system $\mathcal{F}_{S}(G)$ is tamely realized by $G$ if $\kappa_{G}$ is a split surjective (i.e., splits over $\operatorname{Out}\left(\mathcal{L}_{S}^{c}(G)\right)$ ). A fusion system $\mathcal{F}$ is tame if it is tamely realized by some finite group.

For example, if $\mathcal{F}$ is a constrained fusion system and $G$ is a model for $\mathcal{F}$, then $\kappa_{G}$ is an isomorphism by [BMO2, Proposition 1.6] and, hence, $\mathcal{F}$ is tamely realized by $G$.

In section 2.4 we saw the importance of determining whether all simple fusion systems of simple groups are tame and tamely realized by simple groups. For finite groups of Lie type, this was shown in [BMO2]. More precisely, we have the following theorem, where we refer to the introduction to BMO2 for the precise definition of what is meant by "finite group of Lie type".

Theorem 3.5 ([BMO2, Theorems A,B,C]). Let $G$ be a finite group of Lie type in defining characteristic $q$. Then for each prime $p$, the $p$-fusion system $\mathcal{F}$ of $G$ is tame. If $q \neq p$ and the Sylow p-subgroups of $G$ are nonabelian, then $\mathcal{F}$ is tamely realized by another finite group of Lie type in defining characteristic $q^{\prime} \neq p$. If $q=p$, then $\kappa_{G}$ is an isomorphism, except when $G \cong G L_{3}(2)$ or $S z(2) \cong C_{5} \rtimes C_{4}$ (and $p=2$ ).

For example, set $G^{*}=P S L_{2}(9) \cong A_{6}$, fix $S^{*} \in \operatorname{Syl}_{2}\left(G^{*}\right)\left(S^{*} \cong D_{8}\right)$, and set $\mathcal{F}=\mathcal{F}_{S^{*}}\left(G^{*}\right)$ and $\mathcal{L}=\mathcal{L}_{S^{*}}^{c}\left(G^{*}\right)$. Then $\operatorname{Out}(\mathcal{L}) \cong C_{2} \times C_{2} \cong \operatorname{Out}\left(G^{*}\right)$, and $\kappa_{G^{*}}$ is an isomorphism by BLO1, Proposition 7.9]. Hence $\mathcal{F}$ is tame and is tamely realized by $G^{*}$. If $q$ is any prime power such that $q \equiv \pm 7(\bmod 16), G=P S L_{2}(q)$, and $S \in \operatorname{Syl}_{2}(G)$, then $\mathcal{F}_{S}(G) \cong \mathcal{F}$ and $\mathcal{L}_{S}^{c}(G) \cong \mathcal{L}$, so $G$ also realizes $\mathcal{F}$. Also, if $q=q_{0}^{b}$ where $q_{0}$ is a prime, then $\operatorname{Out}(G) \cong C_{b} \times C_{2}$ (the first factor represents the field automorphisms and the second factor the automorphism "transpose inverse"). Hence $\mathcal{F}$ is tamely realized by $G$ only if $q$ is a square.

Tameness for alternating groups and sporadic groups is described in the following theorem.

Theorem 3.6. (a) If $p=2$ and $n \geq 8$, or if $p$ is odd, $n \geq p^{2}$, and $n \equiv 0,1$ $(\bmod p)$, then the $p$-fusion system of $A_{n}$ is tame and $\kappa_{A_{n}}$ is an isomorphism.

(b) If $G$ is a sporadic simple group, then the 2 -fusion system of $G$ is tame. Also, $\kappa_{G}$ is an isomorphism except when $G \cong M_{11}$, in which case Out $(G)=1$, while $\left|\operatorname{Out}\left(\mathcal{L}_{S}^{c}(G)\right)\right|=2$ for $S \in \operatorname{Syl}_{2}(G)$. 
(c) If $G$ is a sporadic simple group and $p$ is an odd prime, then the p-fusion system of $G$ is tame. If the p-fusion system is simple, then $\kappa_{G}$ is an isomorphism except when $G \cong$ He and $p=3$.

Proof. Point (a) is shown in [AOV1, Proposition 4.8], and points (b) and (c) in [08, Theorem A].

We began this section by mentioning extensions of fusion systems, and explaining how fusion systems alone are not enough to get a good theory of such extensions. The following theorem describes one way to do this in terms of automorphisms of linking systems.

Theorem 3.7 ([03, Theorem 9]). Fix a saturated fusion system $\mathcal{F}_{0}$ over a finite p-group $S_{0}$, and let $\mathcal{L}_{0}$ be a (centric) linking system associated to $\mathcal{F}_{0}$. Set $\Gamma_{0}=$ $\operatorname{Aut}_{\mathcal{L}_{0}}\left(S_{0}\right)$, and regard $S_{0}$ as a subgroup of $\Gamma_{0}$ via the structure map $\delta_{S_{0}}: S_{0} \longrightarrow$ $\operatorname{Aut}_{\mathcal{L}_{0}}\left(S_{0}\right)$. Thus $S_{0}=O_{p}\left(\Gamma_{0}\right)$, since $\Gamma_{0} / S_{0} \cong \operatorname{Aut}_{\mathcal{F}_{0}}\left(S_{0}\right) / \operatorname{Inn}\left(S_{0}\right)$ has order prime to $p$ (see Definition 1.2). Fix a finite group $\Gamma$ such that $\Gamma_{0} \unlhd \Gamma$, and a homomorphism $\tau: \Gamma \longrightarrow \operatorname{Aut}\left(\mathcal{L}_{0}\right)$ which makes both triangles in the following diagram commute.

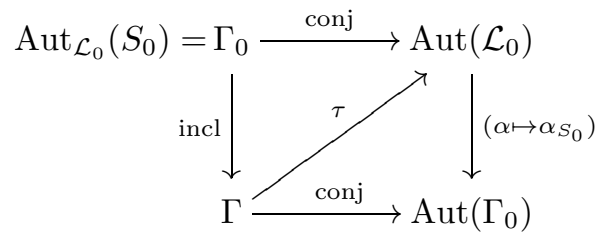

Set $\mathcal{F}_{1}=\left\langle\mathcal{F}_{0}, \operatorname{Aut}_{\Gamma}\left(S_{0}\right)\right\rangle$ as a fusion system over $S_{0}$ (not necessarily saturated), where $\Gamma$ acts on $S_{0}=O_{p}\left(\Gamma_{0}\right) \unlhd \Gamma$ via conjugation. Fix $S \in \operatorname{Syl}_{p}(\Gamma)$. Then there is a saturated fusion system $\mathcal{F}$ over $S$ which contains $\mathcal{F}_{1}$ as a full subcategory and such that $\mathcal{F}_{0} \unlhd \mathcal{F}$.

Theorem 3.7 as stated here is not very satisfactory in that linking systems are needed as part of the input, but the only output is an extension of fusion systems. In fact, the above statement is a shortened version of the main theorem in $\mathrm{O} 3$, where conditions are also given to be able to extend $\mathcal{L}_{0}$ to a linking system $\mathcal{L}$ associated to $\mathcal{F}$ and containing $\mathcal{L}_{0}$ as a normal linking subsystem in the sense of [O3, Definition 8 ] or [AOV1, Definition 1.27]. More generally, the proof of the theorem as stated above involves constructing (in all cases) a transporter system $\mathcal{T}$ associated to $\mathcal{F}$ such that $\mathcal{L}_{0} \unlhd \mathcal{T}$; this is stated and proven explicitly in [BLO5, Theorem 5.4].

3.4. The existence and uniqueness of linking systems. So far, we have sketched the definition of a centric linking system "associated to" a saturated fusion system, without saying why there should be one, and if there is, whether it is unique. The existence and uniqueness of linking systems was proven by Chermak Ch, with some later simplifications in the argument due to Oliver [O4] and Glauberman and Lynd GbL].

Theorem 3.8. Let $\mathcal{F}$ be a saturated fusion system over a p-group $S$. Then the following hold.

(a) There is a centric linking system $\mathcal{L}$ associated to $\mathcal{F}$, and it is unique up to isomorphism of linking systems. 
(b) The homomorphism $\mu_{\mathcal{L}}: \operatorname{Aut}(\mathcal{L}) \longrightarrow \operatorname{Aut}(\mathcal{F})$ is surjective, and it is an isomorphism if $p$ is odd. If $p=2$, then $\operatorname{Ker}\left(\mu_{\mathcal{L}}\right)$ is an abelian 2-group.

Proof. Let $\mathcal{F}^{c} \subseteq \mathcal{F}$ be the full subcategory whose objects are the $\mathcal{F}$-centric subgroups of $S$. Let $\mathcal{O}\left(\mathcal{F}^{c}\right)$ be the orbit category of $\mathcal{F}^{c}$, the category with the same objects, but where $\operatorname{Mor}_{\mathcal{O}\left(\mathcal{F}^{c}\right)}(P, Q)=\operatorname{Inn}(Q) \backslash \operatorname{Hom}_{\mathcal{F}}(P, Q)$. There is a functor

$$
\mathcal{Z}_{\mathcal{F}}: \mathcal{O}\left(\mathcal{F}^{c}\right)^{\mathrm{op}} \longrightarrow \mathrm{Ab}
$$

which sends $P$ to $C_{S}(P)=Z(P)$.

We can regard a centric linking system $\mathcal{L}$ as an extension of the form

$$
1 \longrightarrow\{P\}_{P \in \mathcal{F}^{c}} \stackrel{\delta}{\longrightarrow} \mathcal{L} \stackrel{\pi}{\longrightarrow} \mathcal{O}\left(\mathcal{F}^{c}\right) \longrightarrow 1,
$$

and study the obstructions to the existence and uniquenss of $\mathcal{L}$ by analogy to the obstruction theory for extensions of groups with nonabelian kernel (see McL, Theorems IV.8.7-8]). Thus as shown in [BLO2, Proposition 3.1], the obstruction to the existence of a centric linking system associated to $\mathcal{F}$ lies in $H^{3}\left(\mathcal{O}\left(\mathcal{F}^{c}\right) ; \mathcal{Z}_{\mathcal{F}}\right)$, and the obstruction to its uniqueness lies in $H^{2}\left(\mathcal{O}\left(\mathcal{F}^{c}\right) ; \mathcal{Z}_{\mathcal{F}}\right)$. Also, the obstruction to $\mu_{\mathcal{L}}$ being surjective lies in $H^{2}\left(\mathcal{O}\left(\mathcal{F}^{c}\right) ; \mathcal{Z}_{\mathcal{F}}\right)$, while its kernel is isomorphic to $H^{1}\left(\mathcal{O}\left(\mathcal{F}^{c}\right) ; \mathcal{Z}_{\mathcal{F}}\right)$ (see AKO, Proposition III.5.12]).

In the main theorem of $\mathrm{Ch}$, Chermak proved (a) directly, without reference to the obstruction theory, via a step-by-step construction of the linking system. In O4, Oliver showed how to modify Chermak's argument to prove that $H^{i}\left(\mathcal{O}\left(\mathcal{F}^{c}\right) ; \mathcal{Z}_{\mathcal{F}}\right)=0$ for all $i \geq 2$, and for all $i \geq 1$ when $p$ is odd, thus proving (b). However, both of these arguments depended on the classification of finite simple groups. Recently, Glauberman and Lynd GbL have shown how to modify the proof in $\mathrm{O} 4$ to avoid this, thus finishing a proof of the theorem which is independent of the classification.

Theorem 3.8 has been stated only for centric linking systems, but arbitrary linking systems are also unique in the sense that they depend (up to isomorphism) only on the set of objects. Thus if $\mathcal{L}$ and $\mathcal{L}^{*}$ are two linking systems associated to $\mathcal{F}$, and $\mathcal{H}=\operatorname{Ob}(\mathcal{L}) \cap \operatorname{Ob}\left(\mathcal{L}^{*}\right)$, then the full subcategories of $\mathcal{L}$ and $\mathcal{L}^{*}$ with object set $\mathcal{H}$ are isomorphic as linking systems. This follows from Theorem 3.8(a), together with [AKO, Proposition III.4.8], which says that $\mathcal{L}$ and $\mathcal{L}^{*}$ are full subcategories of linking systems $\widehat{\mathcal{L}}$ and $\widehat{\mathcal{L}}^{*}$, unique up to isomorphism, where $\operatorname{Ob}(\widehat{\mathcal{L}})=\operatorname{Ob}\left(\widehat{\mathcal{L}}^{*}\right)$ is the set of $\mathcal{F}$-quasicentric subgroups of $S$ [AKO, Definition III.4.5].

3.5. Partial groups and localities. In the same paper $\mathrm{Ch}$ where he proved Theorem 3.8. Chermak also proposed a new way to look at linking systems and transporter systems, avoiding the language of categories. To motivate this, let $\mathcal{L}$ be a linking system (or a transporter system), and consider the set $\operatorname{Mor}(\mathcal{L}) / \sim$, where $\sim$ is the equivalence relation generated by restriction and extension of morphisms. A pair (or sequence) of elements in this set can be multiplied (composed) if they contain representatives which are composable, but it is not possible to multiply arbitrary pairs. This set contains a copy of the underlying group $S$ (the classes of morphisms $\delta_{S}(g)$ for $\left.g \in S\right)$. It is an example of what Chermak calls a "partial group".

The following definitions are all taken from [Ch, §2]. Fix a set $\mathcal{X}$, and let $\mathbf{W}(\mathcal{X})$ be the free monoid on $\mathcal{X}$ (i.e., the set of words in the elements of $\mathcal{X}$ ). We regard $\mathcal{X}$ itself as a subset of $\mathbf{W}(\mathcal{X})$ (words of length 1 ). An inversion map $\left(x \mapsto x^{-1}\right)$ 
on $\mathcal{X}$ extends to a map on $\mathbf{W}(\mathcal{X})$ in the usual way: $(u v)^{-1}=v^{-1} u^{-1}$ (and is assumed to have order 2 ). A partial group on $\mathcal{X}$ consists of an inversion map on $\mathcal{X}$-a subset $\mathbf{D} \subseteq \mathbf{W}(\mathcal{X})$ with the properties that $\mathcal{X} \subseteq \mathbf{D}$, and $u v \in \mathbf{D}$ implies $u, v \in \mathbf{D}$-together with a "multiplication" map $\mu: \mathbf{D} \longrightarrow \mathcal{X}$ which satisfies (for $u, v, w \in \mathbf{W}(\mathcal{X}))$ :

- $\left.\pi\right|_{\mathcal{X}}=\operatorname{Id}_{\mathcal{X}}$

- $u v w \in \mathbf{D}$ implies $u \cdot \pi(v) \cdot w \in \mathbf{D}$ and $\pi(u v w)=\pi(u \pi(v) w)$, and

- $u \in \mathbf{D}$ implies $u^{-1} u \in \mathbf{D}$ and $\pi\left(u^{-1} u\right)=1$ (where $1=\pi(\varnothing)$ ).

A partial group $(\mathcal{X}, \mathbf{D}, \pi)$ is a group if $\mathbf{D}=\mathbf{W}(\mathcal{X})$. A subset $\mathcal{Y} \subseteq \mathcal{X}$ is a subgroup if $\mathcal{Y}$ is stable under inversion $\left(y \in \mathcal{Y}\right.$ implies $\left.y^{-1} \in \mathcal{Y}\right)$, and $\mathbf{W}(\mathcal{Y}) \subseteq \mathbf{D}$. If $\mathcal{X}$ is a partial group, $H \leq \mathcal{X}$ is a subgroup of $\mathcal{X}$, and $x \in \mathcal{X}$, then $H^{x}=\left\{\pi\left(x^{-1} h x\right) \mid h \in\right.$ $H\}$ if all such products are defined in $\mathcal{X}$.

When $\mathcal{X}$ is a partial group and $\Delta$ is a set of subgroups of $\mathcal{X}$, let $\mathbf{D}_{\Delta} \subseteq \mathbf{W}(\mathcal{X})$ be the set of all words $w=\left(x_{1}, \ldots, x_{n}\right)$ such that there are subgroups $H_{0}, \ldots, H_{n} \in \Delta$ for which $\left(H_{i-1}\right)^{x_{i}}=H_{i}$ for each $1 \leq i \leq n$. The pair $(\mathcal{X}, \Delta)$ is an objective partial group if $\mathbf{D}=\mathbf{D}_{\Delta}$ and the following holds: whenever $H, K \in \Delta, K_{0} \leq K$, and $x \in \mathcal{X}$ are such that $H^{x} \leq K_{0}$, then $K_{0} \in \Delta$. Finally, a locality consists of a finite partial group $\mathcal{L}$, a $p$-subgroup $S \leq \mathcal{L}$, and a set $\Delta$ of subgroups of $S$ such that $(\mathcal{L}, \Delta)$ is objective and such that $S$ is maximal in the poset of finite $p$-subgroups of $\mathcal{L}$.

Above, we sketched how to construct a partial group from a linking system $\mathcal{L}$ (or a transporter system), and this partial group is in fact a locality (where $\Delta=\operatorname{Ob}(\mathcal{L})$ ). Chermak went on to show in $[\mathrm{Ch}$, Appendix A] that in fact localities are equivalent to transporter systems: given a locality $\mathcal{L}$, one can construct a transporter system with its set of objects as objects of the category.

\section{INTERACTIONS WITH HOMOTOPY THEORY}

The group theoretic properties of a discrete group $G$ are encoded in its classifying space. By a recent theorem, originally conjectured by Martino and Priddy, the $p$ local properties of a finite group $G$ are encoded in its $p$-completed classifying space, where $p$-completion of a space is in the sense of Bousfield and Kan. This is just one of several results which show a close connection between algebraic properties of a group or a saturated fusion system and homotopy theoretic properties of its ( $p$-completed) classifying space.

We begin this section with a brief summary covering classifying spaces of (discrete) groups, $p$-completion of spaces, and geometric realizations of categories. We then state the Martino-Priddy conjecture and describe how it follows as a special case of Chermak's theorem (Theorem 3.8(a)). Afterwards, we define the classifying space of a fusion system $\mathcal{F}$ to be the $p$-completed geometric realization $|\mathcal{L}|_{p}^{\wedge}$ of a linking system $\mathcal{L}$ associated to $\mathcal{F}$, and describe some of its very nice homotopy properties which mimic those already known for $p$-completed classifying spaces of finite groups. We then briefly discuss classifying spectra of fusion systems and, in particular, the characterization by Ragnarsson and Stancu of saturated fusion systems over $S$ in terms of stable summands of $B S$. We end with an application of homotopy theory to proving isomorphisms between certain fusion systems of finite groups of Lie type. 
4.1. Classifying spaces of finite groups and p-completion. A classifying space for a discrete group $G$ is a path connected Hausdorff space $X$ such that $\pi_{1}(X) \cong G$ and the universal covering space $E$ of $X$ is simply connected. Implicit in this definition is the assumption that $X$ has the nice, local properties which imply the existence of a universal covering space.

Equivalently, one can define a universal space for $G$ to be a contractible space $E$ with a proper, free $G$-action: an action of $G$ such that for each $x \in E$, there is an open neighborhood $U$ of $x$ whose translates are pairwise disjoint $(g \neq h$ in $G$ implies $g(U) \cap h(U)=\varnothing)$. A classifying space for $G$ is then a space of the form $X=E / G$, where $E$ is a universal space for $G$. The simplest examples of such spaces arise when $G=\mathbb{Z}$ : the real line $\mathbb{R}$ is a universal space for $\mathbb{Z}$ (where $\mathbb{Z}$ acts by translation), and $\mathbb{R} / \mathbb{Z}$ is a classifying space (homeomorphic to the circle).

A slightly more complicated example is given by the finite cyclic groups. Fix $n>1$, and let $\mu_{n}<\mathbb{C}^{\times}$be the group of $n$th roots of unity. Let $S\left(\mathbb{C}^{m}\right)$ be the unit sphere in $\mathbb{C}^{m}$ (under the $l^{2}$-norm), regard $S\left(\mathbb{C}^{m}\right)$ to be a subspace of $S\left(\mathbb{C}^{m+1}\right)$ in the usual way (by adding final coordinate 0 ), and let $S^{\infty}=\bigcup_{m=1}^{\infty} S\left(\mathbb{C}^{m}\right)$. Then $S^{\infty}$ is contractible and $\mu_{n}$ acts properly and freely on it by multiplication (coordinatewise). Thus $S^{\infty}$ is a universal space, and $B \mu_{n}=S^{\infty} / \mu_{n}$ is a classifying space, for the cyclic group $\mu_{n}$.

In general, we write $B G$ to denote a classifying space for $G$, although this notation is often used for some "canonical" classifying space defined for all discrete groups. We give one example of a canonical classifying space in below (see Example 4.1). The name "classifying space" was originally used for certain spaces which classify real or complex vector bundles. A classifying space $B G$ for a discrete group $G$ classifies homomorphisms to $G$ in the following sense (see, e.g., AKO, Proposition III.1.5(a)]):

Under appropriate topological conditions on $B G$ and another space $X$ (e.g., that both are cell complexes), the set $\operatorname{Hom}\left(\pi_{1}(X), G\right)$ is in bijective correspondence with $[X, B G]_{*}$ : the set of homotopy classes of basepoint preserving continuous maps $X \longrightarrow B G$.

The classifying space of a group $G$ clearly contains complete information about $G$ in its fundamental group. What we are interested in here is a space which gives information only about the $p$-local structure of $G$, and this is where $p$-completion of spaces comes in to the picture. The idea of $p$-completing a space originated with Sullivan $[\mathrm{Su}$, Chapter 3], who defined the $p$-completion of a simply connected space $X$ to be a certain space $X_{p}^{\wedge}$ such that $\pi_{i}\left(X_{p}^{\wedge}\right) \cong \pi_{i}(X) \otimes \widehat{\mathbb{Z}}_{p}$ for each $i$ $[\mathrm{Su}$, Proposition 3.18]. However, this definition worked only for simply connected spaces (later extended to nilpotent spaces) and, in particular, could not be applied to classifying spaces of finite groups.

The $p$-completion under discussion here is that of Bousfield and Kan [BK]. It is a functor $\left(X \mapsto X_{p}^{\wedge}\right)$ from spaces to spaces, together with maps of spaces $\phi_{X}: X \longrightarrow$ $X_{p}^{\wedge}$ which are natural in $X$. Rather than define $p$-completion here, we list a few of its properties. In all cases, the spaces are assumed to be path connected.

- For each map of spaces $f: X \longrightarrow Y$, the induced map $f_{p}^{\wedge}: X_{p}^{\wedge} \longrightarrow Y_{p}^{\wedge}$ is a homotopy equivalence if and only if $H^{*}\left(f ; \mathbb{F}_{p}\right): H^{*}\left(Y ; \mathbb{F}_{p}\right) \longrightarrow H^{*}\left(X ; \mathbb{F}_{p}\right)$ is an isomorphism.

- If $\pi_{1}(X)$ is finite, then $H^{*}\left(\phi_{X} ; \mathbb{F}_{p}\right)$ is an isomorphism, and hence $\left(X_{p}^{\wedge}\right)_{p}^{\wedge} \simeq$ $X_{p}^{\wedge}$. 
- If $\pi_{1}(X)$ is finite, then $\phi_{X}$ is universal in the following sense: if $f: X \longrightarrow Y$ is any map such that $H^{*}\left(f ; \mathbb{F}_{p}\right)$ is an isomorphism, then there is a map $g: Y \longrightarrow X_{p}^{\wedge}$, unique up to homotopy, such that $g \circ f \simeq \phi_{X}$.

- If $\pi_{1}(X)$ is finite, then $\pi_{1}\left(X_{p}^{\wedge}\right) \cong \pi_{1}(X) / O^{p}\left(\pi_{1}(X)\right)$ : the largest quotient group of $\pi_{1}(X)$ which is a $p$-group.

Thus $X_{p}^{\wedge}$ is a space which encodes the properties of $X$ depending only on $H^{*}\left(X ; \mathbb{F}_{p}\right)$.

We refer to $B \mathrm{BK}$ for detailed definitions and properties of $p$-completion, and to AKO, $\S$ III.1.4] for a very brief survey of the subject (with more precise references to $[\mathrm{BK}])$.

4.2. The geometric realization of a category. The connection between the linking systems discussed in the last section and homotopy theory arises via the geometric realization of a small category. Very roughly, when $\mathcal{C}$ is a small category, its geometric realization is a space (or cell complex) $|\mathcal{C}|$ which is a union of one vertex $(c)$ for each object $c$ in $\mathcal{C}$, one edge (copy of the unit interval) $(\varphi)$ for each morphism $\varphi$ in $\mathcal{C}$, one 2 -simplex (triangle) for each pair $(c \rightarrow d \rightarrow e)$ of composable morphisms, and so on in higher dimensions. If $\varphi \in \operatorname{Mor}_{\mathcal{C}}(c, d)$, then the edge $(\varphi)$ has endpoints $(c)$ and $(d)$. For each sequence $(c \stackrel{\varphi}{\rightarrow} d \stackrel{\psi}{\rightarrow} e)$ in $\mathcal{C}$, the corresponding triangle has vertices $(c),(d)$, and $(e)$, and edges $(\varphi),(\psi)$, and $(\psi \circ \varphi)$. In addition, the edge $\left(\operatorname{Id}_{c}\right)$ is identified with the vertex $(c)$, and similarly in higher dimensions. For a more detailed definition, we refer to [Sg, §1-2], or to [AKO, § III.2].

For example, if $\mathcal{C}$ is a category with one object $c$ and one morphism $\operatorname{Id}_{c}$, then $|\mathcal{C}|$ is a point: the simplex corresponding to each sequence $c \stackrel{\mathrm{Id}_{c}}{\longrightarrow} c \cdots \stackrel{\mathrm{Id}_{c}}{\longrightarrow} c$ is identified with the vertex $(c)$. More generally, for $n>0$, let $[n]$ denote the category with $\mathrm{Ob}([n])=\{0,1, \ldots, n\}$, and with a unique morphism $i \rightarrow j$ whenever $i \leq j$. Then $|[1]|$ is an interval, $|[2]|$ is a triangle, and more generally, $|[n]|$ is an $n$-simplex for each $n$.

Example 4.1. Let $G$ be a discrete group. Define categories $\mathcal{B}(G)$ and $\mathcal{E}(G)$ as follows. The category $\mathcal{B}(G)$ contains just one object $o_{G}$, and $\operatorname{Hom}_{\mathcal{B}(G)}\left(o_{G}, o_{G}\right)=G$, where composition is defined by group multiplication (and $g h$ is the composite $h$ followed by $g$ ). Also, $\operatorname{Ob}(\mathcal{E}(G))=G$, and there is a unique morphism $g \rightarrow h$ in $\mathcal{E}(G)$ for each $g, h \in G$. Let $\pi: \mathcal{E}(G) \longrightarrow \mathcal{B}(G)$ be the functor which sends each object of $\mathcal{E}(G)$ to the unique object $o_{G}$, and which sends a morphism $(g \rightarrow h)$ to $h g^{-1}$. Then $|\mathcal{E}(G)|$ is contractible, $G$ acts freely on $|\mathcal{E}(G)|$ via right multiplication, and $\pi$ induces a homeomorphism $|\mathcal{E}(G)| / G \cong|\mathcal{B}(G)|$. Hence $|\mathcal{E}(G)|$ is a universal space for $G$, and $|\mathcal{B}(G)|$ is a classifying space for $G$.

It is this canonical classifying space $|\mathcal{B}(G)|$ which we call $B G$, when we want to refer to a specific space. Note, however, that $|\mathcal{B}(\mathbb{Z})|$, while homotopy equivalent to the circle $S^{1}$, is an infinite dimensional space with structure much more complicated than that of $S^{1}$.

4.3. The Martino-Priddy Conjecture. The link between $p$-local structure of finite groups and $p$-completions of classifying spaces first appeared as a conjecture by Martino and Priddy, now a theorem. Roughly, it says that $G$ and $H$ have the same $p$-local structure if and only if their classifying spaces do so. Here and later it will be convenient to use the notation $G \sim_{p} H$ to mean that $\mathcal{F}_{S}(G) \cong$ $\mathcal{F}_{T}(H)$ (isomorphic as fusion systems) for some $S \in \operatorname{Syl}_{p}(G)$ and $T \in \operatorname{Syl}_{p}(H)$. 
Equivalently, $G \sim_{p} H$ if there is a fusion preserving isomorphism between Sylow $p$-subgroups of $G$ and $H$ (see section 1.2 for the definition of "fusion preserving").

Theorem 4.2 (Martino-Priddy Conjecture). Fix a prime $p$. Let $G$ and $H$ be two finite groups with Sylow p-subgroups $S \leq G$ and $T \leq H$. Then $B G_{p}^{\wedge} \simeq B H_{p}^{\wedge}$ if and only if $G \sim{ }_{p} H$.

Proof. The "only if" part of Theorem $4.2\left(B G_{p}^{\wedge} \simeq B H_{p}^{\wedge}\right.$ implies $\left.G \sim_{p} H\right)$ was proven by Martino and Priddy $[\mathrm{MP}$. The converse was originally proven by Oliver O1, O2, but via a long, case-by-case argument using the classification of finite simple groups.

By [BLO1, Proposition 1.1], $B G_{p}^{\wedge} \simeq\left|\mathcal{L}_{S}^{c}(G)\right|_{p}^{\wedge}$, and similarly for $H$. Thus $B G_{p}^{\wedge} \simeq$ $B H_{p}^{\wedge}$ if $\mathcal{L}_{S}^{c}(G) \cong \mathcal{L}_{T}^{c}(H)$. Thus the "if" part of the theorem also follows as a special case of Chermak's theorem (Theorem 3.8); and by the work of Glauberman and Lynd GbL, the proof of Theorem 4.2 is now independent of the classification.

A theorem of Cartan and Eilenberg from the 1950s implies (as a special case) that if $G \sim_{p} H$ (i.e., $G$ and $H$ are $p$-locally equivalent), then $H^{*}\left(G ; \mathbb{F}_{p}\right) \cong H^{*}\left(H ; \mathbb{F}_{p}\right)$. Since $H^{*}\left(B G_{p}^{\wedge} ; \mathbb{F}_{p}\right) \cong H^{*}\left(B G ; \mathbb{F}_{p}\right) \cong H^{*}\left(G ; \mathbb{F}_{p}\right)$ and similarly for $H$, the "if" part of Theorem 4.2 can be thought of as a refinement of this result.

4.4. Classifying spaces of fusion systems. As was just explained, the main tool used to attack the Martino-Priddy Conjecture was the linking system $\mathcal{L}_{S}^{c}(G)$ of a group $G$. The importance of the linking system in this context arises from the homotopy equivalence $\left|\mathcal{L}_{S}^{c}(G)\right|_{p}^{\wedge} \simeq B G_{p}^{\wedge}$ (for each finite group $G$ with $S \in \operatorname{Syl}_{p}(G)$ ) shown in [BLO1, Proposition 1.1].

Motivated by this equivalence, a classifying space of a saturated fusion system $\mathcal{F}$ is defined to be the $p$-completed geometric realization $|\mathcal{L}|_{p}^{\wedge}$ of a linking system associated to $\mathcal{F}$. These classifying spaces no longer classify anything (that we know of); but they do have many of the very nice homotopy properties of the $p$-completed classifying spaces of finite groups, as described in section 4.6 below. In particular, the $\bmod p$ cohomology of a classifying space of $\mathcal{F}$ is what one would predict, by analogy with the Cartan-Eilenberg formula for $H^{*}\left(G ; \mathbb{F}_{p}\right)$ in terms of its $p$-fusion.

This brings us back to the predictions by Benson in Be2. Motivated by certain spaces which had the cohomology that Solomon's "groups" would have if they existed, he predicted that it should be possible to associate classifying spaces to abstract fusion systems, defined at that time only in Puig's unpublished notes. Benson also predicted (in an unpublished manuscript) that they should arise as realizations of categories: categories with properties which linking systems are now known to have. That the spaces constructed by Benson, based on the DwyerWilkerson space $B D I(4)$, are actually homotopy equivalent to classifying spaces (in the above sense) for the fusion systems $\operatorname{Sol}(q)$ was confirmed by Levi and Oliver in [LO, Theorem 4.5].

4.5. Classifying spaces of fusion systems as homotopy rigidifications. Classifying spaces of fusion systems can also be characterized without mentioning linking systems, in a way first proposed by Benson (unpublished), a way which perhaps seems more natural from the point of view of homotopy theory. Recall the orbit category of a saturated fusion system $\mathcal{F}$ over $S$, already defined in the proof of Theorem 3.8. We let $\mathcal{F}^{c} \subseteq \mathcal{F}$ be the full subcategory whose objects are the $\mathcal{F}$ centric subgroups of $S$, and let $\mathcal{O}\left(\mathcal{F}^{c}\right)$ be the category with the same objects (the 
$\mathcal{F}$-centric subgroups of $S$ ), and with morphism sets

$$
\operatorname{Mor}_{\mathcal{O}\left(\mathcal{F}^{c}\right)}(P, Q)=\operatorname{Inn}(Q) \backslash \operatorname{Hom}_{\mathcal{F}}(P, Q) .
$$

Thus, for example, $\operatorname{Aut}_{\mathcal{O}(\mathcal{F} c)}(P) \cong \operatorname{Out}_{\mathcal{F}}(P)$.

Let Top be the category of topological spaces and continuous maps. Let hoTop be the homotopy category: the category whose objects are topological spaces, and where $\operatorname{Mor}_{\text {hoTop }}(X, Y)$ is the set of homotopy classes of maps from $X$ to $Y$. Let $h:$ Top $\longrightarrow$ hoTop be the natural functor. There is a functor $B: \mathcal{O}\left(\mathcal{F}^{c}\right) \longrightarrow$ hoTop which sends an object $P \leq S$ to the space $B P$, and which sends a morphism $[\varphi] \in$ $\operatorname{Mor}_{\mathcal{O}\left(\mathcal{F}^{c}\right)}(P, Q)$ (for $\left.\varphi \in \operatorname{Hom}_{\mathcal{F}}(P, Q)\right)$ to the homotopy class of $B \varphi: B P \longrightarrow B Q$. Since $B \alpha \simeq \operatorname{Id}_{B P}$ for $\alpha \in \operatorname{Inn}(P)$, this does give a well defined functor from the orbit category to the homotopy category.

A rigidification of the homotopy functor $B$ is a lifting of $B$ to a functor $\widetilde{B}: \mathcal{O}\left(\mathcal{F}^{c}\right) \longrightarrow$ Top. More precisely, a rigidification consists of the functor $\widetilde{B}$, together with a natural isomorphism of functors from $h \circ \widetilde{B}$ to $B$. Once we have found a rigidification $\widetilde{B}$, we can define a "classifying space"

$$
B \mathcal{F} \stackrel{\text { def }}{=} \operatorname{hocolim}(\widetilde{B}):
$$

the homotopy colimit (see, e.g., AKO, $\S$ III.5.5]) of the functor $\widetilde{B}: \mathcal{O}\left(\mathcal{F}^{c}\right) \longrightarrow$ Top.

As described in [BLO2, Proposition 2.2], each centric linking system $\mathcal{L}$ associated to $\mathcal{F}$ determines a rigidification $\widetilde{B}_{\mathcal{L}}$ of $B$, constructed by taking the left homotopy Kan extension of the constant "point" functor $\mathcal{L} \stackrel{*}{\longrightarrow}$ Top along the projection $\tilde{\pi}: \mathcal{L} \longrightarrow \mathcal{O}\left(\mathcal{F}^{c}\right)$. Furthermore, by the same proposition, the homotopy colimit of $\widetilde{B}_{\mathcal{L}}$ is homotopy equivalent to $|\mathcal{L}|$. In fact, by BLO3, Proposition 4.6], this correspondence defines a bijection between equivalence classes of rigidifications of $B$ and isomorphism classes of linking systems, and hence between classifying spaces of $\mathcal{F}$ defined via linking systems and those defined via rigidifications.

There is an obstruction theory due to Dwyer and Kan [DK] to the existence and uniqueness of rigidifications of functors to the homotopy category. Upon applying this theory to the homotopy functor $B$, we find exactly the same obstruction groups $H^{i}\left(\mathcal{O}\left(\mathcal{F}^{c}\right) ; \mathcal{Z}_{\mathcal{F}}\right)(i=3,2)$ as those described in the proof of Theorem 3.8 to the existence and uniqueness of linking systems. So even without mentioning linking systems at all, the obstruction theory behind Chermak's theorem (Theorem 3.8) can be applied to prove the existence and uniqueness of classifying spaces associated via rigidifications to a saturated fusion system $\mathcal{F}$.

4.6. Homotopy properties of $|\mathcal{L}|_{p}^{\wedge}$. One of the consequences of the Sullivan conjecture, proven in slightly different forms by Carlsson, Lannes, and Miller in the early $1980 \mathrm{~s}$, was that the $p$-completed classifying spaces of finite groups have some remarkable, unusual homotopy theoretic properties. From the point of view of homotopy theorists, one of the reasons for interest in fusion systems was that their classifying spaces have similar, very nice properties.

These properties are summarized in Table 2, In that table, for spaces $X$ and $Y$, $[X, Y]$ denotes the set of homotopy classes of continuous maps from $X$ to $Y$, and $\operatorname{map}(X, Y)_{f}$ is the connected component of $f: X \rightarrow Y$ in the space of all maps. Also (and less standard), Out $(X)$ denotes the group of homotopy classes of self homotopy equivalences of $X$; i.e., the group of invertible elements in the monoid $[X, X]$. The first column in Table 2 lists properties of $B G_{p}^{\wedge}$ for a finite group $G$, 
TABLE 2

\begin{tabular}{|c|c|}
\hline \multicolumn{1}{|c|}{$B G_{p}^{\wedge}$} & $(S, \mathcal{F}, \mathcal{L})$ \\
\hline \hline$H^{*}\left(B G ; \mathbb{F}_{p}\right) \cong \lim _{\mathcal{F}_{S}(G)} H^{*}\left(-; \mathbb{F}_{p}\right)$ & $H^{*}\left(|\mathcal{L}| ; \mathbb{F}_{p}\right) \cong \lim _{\mathcal{F}} H^{*}\left(-; \mathbb{F}_{p}\right)$ \\
\hline $\begin{array}{l}\text { for each } p \text {-group } Q, \\
{\left[B Q, B G_{p}^{\wedge}\right] \cong \operatorname{Hom}(Q, G) / \operatorname{Inn}(G)}\end{array}$ & $\begin{array}{l}\text { for each } p \text {-group } Q, \\
{\left[B Q,|\mathcal{L}|_{p}^{\wedge}\right] \cong \operatorname{Hom}(Q, S) /(\mathcal{F} \text {-fusion })}\end{array}$ \\
\hline $\begin{array}{l}\text { for each } p \text {-group } Q, \\
\text { for each } \rho \in \operatorname{Hom}(Q, G), \\
\operatorname{map}\left(B Q, B G_{p}^{\wedge}\right)_{B \rho} \simeq B C_{G}(\rho(Q))_{p}^{\wedge}\end{array}$ & $\begin{array}{l}\text { for each } p \text {-group } Q, \text { for each }(Q, S) \text { with } \rho(Q) \text { fully centr., } \\
\operatorname{map}\left(B Q,|\mathcal{L}|_{p}^{\wedge}\right)_{B \rho} \simeq\left|C_{\mathcal{L}}(\rho(Q))\right|_{p}^{\wedge}\end{array}$ \\
\hline $\operatorname{Out}\left(B G_{p}^{\wedge}\right) \cong \operatorname{Out}\left(\mathcal{L}_{S}^{c}(G)\right)$ & $\operatorname{Out}\left(|\mathcal{L}|_{p}^{\wedge}\right) \cong \operatorname{Out}(\mathcal{L})$ \\
\hline
\end{tabular}

while the second column compares those to properties of $|\mathcal{L}|_{p}^{\wedge}$ when $\mathcal{L}$ is a centric linking system associated to a saturated fusion system $\mathcal{F}$ over a $p$-group $S$.

For groups, the isomorphisms in Table 2 are shown in [CE, Theorem XII.10.1], [BL, Proposition 2.1], and BLO1, Theorem 4.5], respectively. For fusion systems, they are shown in Theorems B, C, and D (or 8.1) of [BLO2].

The description of $H^{*}\left(|\mathcal{L}| ; \mathbb{F}_{p}\right)$ as an inverse limit (equivalently, as a group of "stable elements" in $H^{*}\left(S ; \mathbb{F}_{p}\right)$ ) can easily be extended to (untwisted) coefficients in an arbitary finite $p$-group [BCGLO2, Lemma 6.12]. Very recently, Molinier, in an as-yet unpublished work, has extended this to certain cases of coefficients twisted by an action of $\pi_{1}(|\mathcal{L}|)$, while giving examples to show that twisted cohomology cannot always be computed in this way.

Another curious result was shown in BLO2, Theorem 7.4]: a triple $(S, \mathcal{F}, \mathcal{L})$ is determined (up to isomorphism) by the space $|\mathcal{L}|_{p}^{\wedge}$ (up to homotopy). In other words, if $\mathcal{F}$ and $\mathcal{F}^{*}$ are saturated fusion systems over $p$-groups $S$ and $S^{*}$, with associated centric linking systems $\mathcal{L}$ and $\mathcal{L}^{*}$, respectively, and $|\mathcal{L}|_{p}^{\wedge}$ is homotopy equivalent to $\left|\mathcal{L}^{*}\right|_{p}^{\wedge}$, then $(S, \mathcal{F}, \mathcal{L}) \cong\left(S^{*}, \mathcal{F}^{*}, \mathcal{L}^{*}\right)$. In other words, $S \cong S^{*}, \mathcal{F} \cong \mathcal{F}^{*}$, and $\mathcal{L} \cong \mathcal{L}^{*}$, via isomorphisms which preserve all of the structure maps between these categories.

4.7. Fusion systems and stable homotopy. This subsection is much more specialized than most in this survey, and we assume that readers are already familiar with the use of spectra in algebraic topology. We describe a characterization, due to Ragnarsson and Stancu, of saturated fusion systems over $S$ in terms of stable summands of $B S$.

When $\mathcal{F}$ is a saturated fusion system over a $p$-group $S$ and $\mathcal{L}$ is a centric linking system associated to $\mathcal{F}$, the suspension spectrum $\mathbb{B} \mathcal{F} \stackrel{\text { def }}{=} \Sigma^{\infty}|\mathcal{L}|_{p}^{\wedge}$ can be regarded as a "classifying spectrum" for $\mathcal{F}$. But in fact, as shown by Ragnarsson Rg, this can be constructed independently of the linking system, in terms of a certain idempotent endomorphism of $\mathbb{B} S=\Sigma^{\infty} B S_{p}^{\wedge}$.

Let $A(S, S)$ be the Grothendieck group on the monoid of isomorphism classes of $(S, S)$-bisets for which the left action is free. As an abelian group, $A(S, S)$ has as additive basis the set of isomorphism classes of such bisets on which $S \times S$ acts 
transitively. It also has a ring structure, where multiplication is defined via direct product over $S:[X] \cdot[Y]=\left[X \times{ }_{S} Y\right]$, where $X \times{ }_{S} Y=(X \times Y) /\langle(x s, y) \sim(x, s y)\rangle$. A biset (or an element of $A(S, S)$ ) is symmetric if it remains unchanged after exchanging the two $S$-actions.

For example, for each $P \leq S$ and each $\varphi \in \operatorname{Hom}(P, S)$, there is a transitive $(S, S)$-biset

$$
[P, \varphi]=(S \times S) /\langle(s, x t) \sim(s \varphi(x), t) \mid s, t \in S, x \in P\rangle
$$

with the left and right actions of $S$ given by $r[s, t] u=[r s, t u]$ (and where $[r, s] \in$ $[P, \varphi]$ is the equivalence class of $(r, s) \in S \times S)$. It is not hard to see that each left-free transitive biset has this form, and one can give explicit conditions for when $[P, \varphi] \cong$ $[Q, \psi]$. There is a natural homomorphism of rings from $A(S, S)_{p}^{\wedge}=A(S, S) \otimes_{\mathbb{Z}} \mathbb{Z}_{p}$ to $\operatorname{End}(\mathbb{B} S)$ which sends $[P, \varphi]$ to the composite map $\mathbb{B} S \stackrel{\text { transfer }}{\longrightarrow} \mathbb{B} P \stackrel{\mathbb{B} \varphi}{\longrightarrow} \mathbb{B} S$.

In [BLO2, Proposition 5.5], for a saturated fusion system $\mathcal{F}$ over $S$, a certain "characteristic $(S, S)$-biset" $\Omega$ was constructed, one which has the property that the mapping telescope of the sequence $\mathbb{B} S \stackrel{[\Omega]}{\longrightarrow} \mathbb{B} S \stackrel{[\Omega]}{\longrightarrow} \mathbb{B} S \longrightarrow \cdots$ has the mod $p$ cohomology of $\mathcal{F}$ and hence can be identified with $\mathbb{B} \mathcal{F}$. In $\mathrm{Rg}$, Ragnarsson makes this procedure more precise and more functorial, by replacing the characteristic biset by a characteristic idempotent in $A(S, S)_{(p)}$. Using this, he constructs a functor from saturated fusion systems to spectra which sends $\mathcal{F}$ to $\mathbb{B} \mathcal{F}$ the mapping telescope for the characteristic idempotent.

Conversely, in $[\mathrm{Rg}$, Theorem A], Ragnarsson gives an explicit algorithm for recovering a saturated fusion system $\mathcal{F}$ from its classifying spectrum $\mathbb{B} \mathcal{F}$ or, rather, from the pair $\left(\mathbb{B} \mathcal{F}, \sigma_{\mathcal{F}}\right)$ where $\sigma_{\mathcal{F}}: \mathbb{B} S \longrightarrow \mathbb{B} \mathcal{F}$ is the projection. In fact, he shows that for $P, Q \leq S, \operatorname{Hom}_{\mathcal{F}}(P, Q)$ is the set of all $\varphi \in \operatorname{Inj}(P, Q)$ such that the following two composite maps are homotopic:

$$
\mathbb{B} P \underset{\mathbb{B i n c l} l_{Q}^{S} \circ \mathbb{B} \varphi}{\stackrel{\mathbb{B i n c l}_{P}^{S}}{\longrightarrow}} \mathbb{B} S \stackrel{\sigma_{\mathcal{F}}}{\longrightarrow} \mathbb{B} \mathcal{F} .
$$

More recently, Ragnarsson and Stancu showed in [RSt, Theorem B] that for each $p$-group $S$, there is a bijective correspondence between saturated fusion systems over $S$, and symmetric idempotents in $A(S, S)_{(p)}$ of augmentation 1 which satisfy a certain Frobenius reciprocity relation: a correspondence which sends a saturated fusion system to its characteristic idempotent. In other words, they give a characterization of saturation for fusion systems over $S$ in terms of the ring $A(S, S)_{(p)}$ and in terms of stable summands of $\mathbb{B} S$ satisfying certain conditions.

4.8. An application: fusion systems of finite groups of Lie type. By Theorem 4.2 (the implication proven by Martino and Priddy), if $G$ and $H$ are finite groups such that $B G_{p}^{\wedge} \simeq B H_{p}^{\wedge}$, then $G$ and $H$ have the same local structure in the sense that $\mathcal{F}_{S}(G) \cong \mathcal{F}_{T}(H)$ for any $S \in \operatorname{Syl}_{p}(G)$ and $T \in \operatorname{Syl}_{p}(H)$. As in section 4.3, we write $G \sim_{p} H$ for this relation.

The following is the main result in BMO1] (Theorem A). In the statement, when $q$ is prime to $p, \overline{\langle q\rangle}$ denotes the closure (in the $p$-adic topology) of the subgroup $\langle q\rangle \leq \mathbb{Z}_{p}^{\times}$. One can think of a reductive group scheme over $\mathbb{Z}$ as a functor from commutative rings to groups satisfying certain conditions; in particular, it sends an algebraically closed field $k$ to an algebraic group over $k$. Examples of group schemes $\mathbb{G}$ relevant to the following theorem include the usual connected classical 
groups $G L_{n}, S L_{n}, P S L_{n}, S p_{2 n}$, as well as the exceptional groups $G_{2}, \ldots, E_{8}$. Note, however, that $S O_{n}$ is not connected in defining characteristic 2.

Theorem 4.3. Fix a prime $p$, a connected reductive group scheme $\mathbb{G}$ over $\mathbb{Z}$, and a pair of prime powers $q$ and $q^{\prime}$ both prime to $p$. Then the following hold.

(a) $\mathbb{G}(q) \sim_{p} \mathbb{G}\left(q^{\prime}\right)$ if $\overline{\langle q\rangle}=\overline{\left\langle q^{\prime}\right\rangle}$.

(b) If $\mathbb{G}$ is of type $A_{n}, D_{n}$, or $E_{6}$ and $\tau$ is a graph automorphism of $\mathbb{G}$, then ${ }^{\tau} \mathbb{G}(q) \sim_{p}{ }^{\tau} \mathbb{G}\left(q^{\prime}\right)$ if $\overline{\langle q\rangle}=\overline{\left\langle q^{\prime}\right\rangle}$.

(c) If the Weyl group of $\mathbb{G}$ contains an element which acts on the maximal torus by inverting all elements, then $\mathbb{G}(q) \sim_{p} \mathbb{G}\left(q^{\prime}\right)$, or ${ }^{\tau} \mathbb{G}(q) \sim_{p}{ }^{\tau} \mathbb{G}\left(q^{\prime}\right)$ for $\tau$ as in (b), if $\overline{\langle-1, q\rangle}=\overline{\left\langle-1, q^{\prime}\right\rangle}$.

(d) If $\mathbb{G}$ is of type $A_{n}, D_{n}$ for $n$ odd, or $E_{6}$, and $\tau$ is a graph automorphism of $\mathbb{G}$ of order two, then ${ }^{\tau} \mathbb{G}(q) \sim_{p} \mathbb{G}\left(q^{\prime}\right)$ if $\overline{\langle-q\rangle}=\overline{\left\langle q^{\prime}\right\rangle}$.

The proof in [BMO1] of this result is carried out by first proving that $B \mathbb{G}(q)_{p}^{\wedge} \simeq$ $B \mathbb{G}\left(q^{\prime}\right)_{p}^{\wedge}$ (in the situation of case $(\mathrm{a})$ ), and then concluding that $\mathbb{G}(q) \sim_{p} \mathbb{G}\left(q^{\prime}\right)$ by Theorem 4.2. To our knowledge, no purely algebraic proof of this result has yet been found.

To make Theorem 4.3 more explicit, we note the following characterization of the condition that $\overline{\langle q\rangle}=\overline{\left\langle q^{\prime}\right\rangle}$ in $\mathbb{Z}_{p}^{\times}$. For each $q$ and $q^{\prime}$ prime to $p$, of order $s$ and $s^{\prime}$, respectively, in $(\mathbb{Z} / p)^{\times}$,

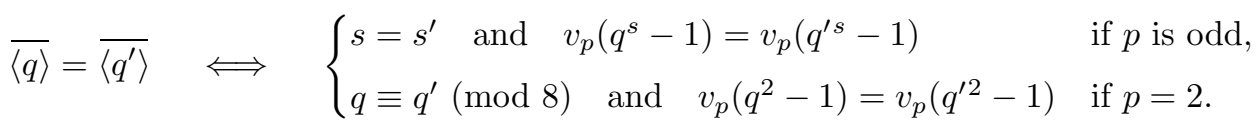

This follows easily from the decompositions

$$
\mathbb{Z}_{p}^{\times}= \begin{cases}(\mathbb{Z} / p)^{\times} \times\left(1+p \mathbb{Z}_{p}\right) \cong(\mathbb{Z} /(p-1)) \times \mathbb{Z}_{p} & \text { if } p \text { is odd } \\ \{ \pm 1\} \times\left(1+4 \mathbb{Z}_{2}\right) & \text { if } p=2 .\end{cases}
$$

\section{INTERACTIONS WITH REPRESENTATION THEORY}

This is the subject which provided Puig's original motivation for defining saturated fusion systems, and it has many connections with the subject. However, since we are unable to really do justice to these connections, we will just give here a brief survey to explain how fusion systems appear in representation theory, and we will indicate a few of the results and questions in this area. Much of what is in this section can be found in $\mathrm{Pg} 5$, and also in [AKO, Part IV].

Fix a finite group $G$ and a prime $p$. Let $k$ be an algebraically closed field of characteristic $p$. A block in $k G$ can be regarded either as an indecomposable 2 -sided ideal in $k G$, or as a primitive (indecomposable) idempotent in the center $Z(k G)$. We will usually use the same symbol to denote the ideal and the idempotent, although we will sometimes write $b \cdot k G$ to denote the ideal when $b \in Z(k G)$ is the idempotent.

After a very brief outline of some basic properties of blocks in $k G$, we define the fusion system of a block in section 5.3 and then list in section 5.4 a few results which illustrate the connection between the fusion system and the representation theory of the block. One example, the gluing conjecture (Conjecture 5.22), is discussed. 
5.1. Vertices of indecomposable $k G$-modules. We start by fixing some notation. Let $H \leq G$ be a pair of finite groups. For each $k H$-module $N, N \uparrow G$ denotes the $k G$-module induced from $N$. For each $k G$-module $M, M \downarrow_{H}$ is the restriction of $M$ to a $k H$-module. If $M_{0}$ and $M$ are two $k G$-modules, then $M_{0} \mid M$ means that $M_{0}$ is isomorphic to a direct summand of $M$.

Again let $M$ be a $k G$-module. Let $M^{G} \leq M^{H}$ be the centralizers of $G$ and $H$, respectively, let

$$
\operatorname{Tr}_{H}^{G}: M^{H} \longrightarrow M^{G}
$$

be the usual trace map $\left(\operatorname{Tr}_{H}^{G}(x)=\sum_{g H \in G / H} g x\right)$, and set $M_{H}^{G}=\operatorname{Im}\left(\operatorname{Tr}_{H}^{G}\right) \leq M^{G}$. For example, when $M=k G$ and $G$ acts by conjugation,

$$
\begin{aligned}
& (k G)^{G}=Z(k G)=\langle((g)) \mid g \in G\rangle, \\
& (k G)_{H}^{G}=\left\langle((g))|g \in G, p \nmid| C_{G}(g): C_{H}(g) \mid\right\rangle, \quad \text { where }((g))=\sum_{g^{\prime} \text { conj. } g} g^{\prime} .
\end{aligned}
$$

Definition-Proposition 5.1 ([Gr, Theorem 1], Be1, Proposition 3.6.4]). For a pair $H \leq G$ of finite groups, a $k G$-module $M$ is called relatively $H$-projective if any of the following three equivalent conditions hold:

(a) $M \mid N \uparrow^{G}$ for some $k H$-module $N$;

(b) $M \mid M \downarrow_{H} \uparrow^{G}$; or

(c) $\operatorname{Id}_{M} \in \operatorname{Aut}(M)_{H}^{G}=\operatorname{Im}\left[\operatorname{Tr}_{H}^{G}: \operatorname{Aut}_{H}(M) \longrightarrow \operatorname{Aut}_{G}(M)\right]$.

Now let $M$ be an indecomposable $k G$-module. A vertex for $M$ is a subgroup $H \leq G$ which is minimal with the property that $M$ is relatively $H$-projective. The following properties of vertices are fundamental.

Proposition 5.2 (Green). Let $M$ be an indecomposable $k G$-module. Then each vertex for $M$ is a p-subgroup of $G$, and all vertices are $G$-conjugate to each other.

Proof. We give here a very brief sketch of the proof and refer for details to Green's original paper [Gr, Theorem 4], or to Alp2, Theorem 9.4.1] or [Be1, Proposition 3.10.2]. First, since $\operatorname{char}(k)=p$, if $K \leq H$ are such that $[H: K]$ is prime to $p$, then $M$ is relatively $K$-projective if it is relatively $H$-projective; this follows from standard averaging techniques.

Let $P, Q \leq G$ be such that $M$ is relatively $P$-projective and relatively $Q$ projective, and such that $P$ has smallest possible order among such subgroups. Thus $M \mid N \uparrow^{G}$ for some $k P$-module $M$, and $M \mid M \downarrow_{Q} \uparrow^{G}$. So by Mackey's double coset formula, $M$ is a direct summand of

$$
\left(N \uparrow^{G} \downarrow_{Q}\right) \uparrow^{G}=\bigoplus_{i=1}^{m} N \downarrow_{H_{i}} \uparrow^{G}
$$

for some subgroups $H_{1}, \ldots, H_{m}$, each of which is contained in $P$ and contained in a subgroup conjugate to $Q$. By the Krull-Schmidt theorem and since $M$ is indecomposable, $M \mid N \downarrow_{H_{i}} \uparrow^{G}$ for some $i$, so $H_{i}=P$ by the minimality assumption on $|P|$, and $P$ is contained in a subgroup conjugate to $Q$.

For example, the vertices of the $k G$-module $k$ with trivial action are the Sylow $p$-subgroups of $G$. More generally, if $M$ is an indecomposable $k G$-module, $H \leq G$ has index prime to $p$, and $k \mid M \downarrow_{H}$, then the Sylow $p$-subgroups of $G$ are vertices for $M$. 
5.2. Blocks in group rings and their defect groups. An idempotent $e$ in an algebra $A$ is primitive if there is no nontrivial decomposition $e=e_{1}+e_{2}$ where $e_{1}$ and $e_{2}$ are idempotents and $e_{1} e_{2}=0=e_{2} e_{1}$. Regard a block in $k G$ as a primitive idempotent $b \in Z(k G)$. We also identify the block with the 2 -sided ideal $b \cdot k G$; thus a block is a minimal direct factor of $k G$ which is a ring.

We regard $k G$ as a $k[G \times G]$-module, via the action $(g, h) \cdot \xi=g \xi h^{-1}$ for $(g, h) \in$ $G \times G$ and $\xi \in k G$. Since $b$ is a central idempotent, $b \cdot k G$ is a $k[G \times G]$-submodule, and is indecomposable since any splitting as a direct sum would lead to a decomposition of the idempotent $b$.

For each $H \leq G$, let $\Delta H=\{(h, h) \mid h \in H\} \leq G \times G$. As $k[G \times G]$-modules, $k G \cong k \uparrow G \times G$, where $k$ has the trivial action of $\Delta G$. Hence $k G$ is relatively $\Delta G$ projective, and so all of its direct summands are also relatively $\Delta G$-projective. So each vertex of an indecomposable direct summand is $(G \times G)$-conjugate to a subgroup of $\Delta G$.

Definition 5.3. Let $b \in Z(k G)$ be a block. A defect group of $b$ is a $p$-subgroup $D \leq G$ such that $\Delta D$ is a vertex of $b \cdot k G$ when regarded as a $k[G \times G]$-module.

Since all vertices of $b \cdot k G$ are conjugate to each other, all defect groups of $b$ are conjugate to each other.

For any $p$-subgroup $P \leq G$, the Brauer map

$$
\operatorname{Br}_{P}: C_{k G}(P) \longrightarrow k C_{G}(P)
$$

is defined by truncation (dropping all terms with elements not in $C_{G}(P)$ ). Since $\operatorname{char}(k)=p$, this is a ring homomorphism.

The following proposition gives three different ways of characterizing defect groups for a block in $k G$.

Proposition 5.4. The following are equivalent for each block $b \in Z(k G)$ and each p-subgroup $D \leq G$ :

(a) $D$ is a defect group for $b$;

(b) $D$ is maximal among $p$-subgroups $P \leq S$ such that $\operatorname{Br}_{P}(b) \neq 0$; and

(c) $D$ is minimal among subgroups $H \leq G$ such that $b \in(k G)_{H}^{G}$.

Proof. (a $\Longleftrightarrow \mathbf{c})$ We must show, for each $H \leq G$, that $b \cdot k G$ is relatively $\Delta H$ projective if and only if $b \in(k G)_{H}^{G}$.

Fix $H \leq G$. In general, a $k G$-module $M$ is relatively $H$-projective if and only if $\operatorname{Id}_{M}$ lies in the image of the trace map $\operatorname{Tr}_{H}^{G}: \operatorname{End}_{H}(M) \longrightarrow \operatorname{End}_{G}(M)$ (see Definition 5.1 or [Be1, Proposition 3.6.4(vii)]). In particular, if the $k[G \times G]$-module $b \cdot k G$ is relatively $\Delta H$-projective, then

$$
\operatorname{Id}_{b \cdot k G} \in \operatorname{End}(b \cdot k G)_{\Delta H}^{G \times G} \subseteq \operatorname{End}(b \cdot k G)_{G \times H}^{G \times G} .
$$

Let $f \in \operatorname{End}_{G \times H}(b \cdot k G)$ be such that $\operatorname{Id}=\operatorname{Tr}_{G \times H}^{G \times G}(f)$; then $\operatorname{Tr}_{H}^{G}(f(b))=b$, and hence $b \in(k G)_{H}^{G}$.

Conversely, if $b=\operatorname{Tr}_{H}^{G}(\xi)$ for some $\xi \in(k G)^{H}$, define $f \in \operatorname{Hom}_{\Delta H}(k G, b \cdot k G)$ by setting $f(1)=\xi$, and $f(g)=0$ for $1 \neq g \in G$. One now checks that $\operatorname{Tr}_{\Delta H}^{G \times G}(f)(x)=$ $b x$ for $x \in k G$, so $\operatorname{Id}_{b \cdot k G} \in \operatorname{End}(b \cdot k G)_{\Delta H}^{G \times G}$, and $b \cdot k G$ is relatively $\Delta H$-projective. 
(b $\Longleftrightarrow \mathbf{a}, \mathbf{c})$ By (11), for each $H \leq G, b \in(k G)_{H}^{G}$ if and only if $p \nmid\left[C_{G}(g): C_{H}(g)\right]$ for all $g \in \operatorname{supp}(b)$. In other words, $b \in(k G)_{H}^{G}$ if and only if $H$ contains a Sylow $p$-subgroup of $C_{G}(g)$ for each $g \in \operatorname{supp}(b)$. By comparison, for each $p$-subgroup $P \leq$ $G, \operatorname{Br}_{P}(b) \neq 0$ if and only if some $g \in \operatorname{supp}(b)$ is contained in $C_{G}(P)$; equivalently, $P \leq C_{G}(g)$. Thus if $P, Q \leq G$ are $p$-subgroups such that $\operatorname{Br}_{P}(b) \neq 0$ and $b \in(k G)_{Q}^{G}$, then $P$ is contained in a subgroup conjugate to $Q$, and in particular, $P$ is contained in some defect group $D$.

To prove that (b) is equivalent to the other two conditions, it remains to show that $\mathrm{Br}_{D}(b) \neq 0$ for each defect group $D$ for $b$. We refer to [Be1, Lemma 6.2.4] for a proof of this.

Thus the defect groups of a block $b$ in $k G$ are the $p$-subgroups $D \leq G$ which satisfy the above three conditions.

The principal block of $k G$ is the block $b_{0}$ for which $k$ (with the trivial action) is a $b_{0} \cdot k G$-module. In other words, $b_{0}$ acts on $k$ by the identity, and thus it has augmentation (the sum of its coefficients) $\epsilon\left(b_{0}\right)=1$. This implies that $\operatorname{Br}_{P}\left(b_{0}\right) \neq 0$ for all $p$-subgroups $P \leq G$ (since $\epsilon\left(x-\operatorname{Br}_{P}(x)\right)=0$ for all $x \in k G^{P}$ ). Thus by Proposition 5.4(b), the defect groups of $b_{0}$ are the Sylow $p$-subgroups of $G$.

When $b$ is a block in $k G$, the vertex of each indecomposable $b \cdot k G$-module is contained in a defect group of $b$ (see Alp2, Theorem 13.5]). Since the vertices of the module $k$ with trivial action are the Sylow $p$-subgroups of $G$, this gives another reason why the defect groups of the principal block in $k G$ are Sylow $p$-subgroups of $G$.

Theorem 5.5 (Brauer's first main theorem Alp2, Theorem 14.2]). If $D$ is a psubgroup of $G$, then there is a one-to-one correspondence

$$
\left\{\begin{array}{c}
\text { blocks of } k G \\
\text { with defect group } D
\end{array}\right\} \cong\left\{\begin{array}{c}
\text { blocks of } k N_{G}(D) \\
\text { with defect group } D
\end{array}\right\}
$$

defined by sending a block idempotent $B$ of $k G$ to $\operatorname{Br}_{D}(B)$. A block $b$ of $k N_{G}(D)$ with defect group $D$ corresponds to the unique block $B$ of $k G$ with defect group $D$ such that $b \mid\left(\left.B\right|_{N(D) \times N(D)}\right)$.

We also note the following.

Lemma 5.6 ([Be1, Proposition 6.2.2]). Assume that $P \unlhd G$ is a normal p-subgroup. Then the following hold:

(a) Each idempotent in $Z(k G)$ lies in $k C_{G}(P)$.

(b) $P$ is contained in the defect groups of each block of $G$.

Proof. By definition, $\operatorname{Ker}\left(\operatorname{Br}_{P}\right) \subseteq \operatorname{Ker}[k G \longrightarrow k[G / P]]$, and this kernel is nilpotent since the augmentation ideal $I \subseteq k P$ is nilpotent (see [Be1, $\S 3.14]$ ). Hence for any idempotent $b \in Z(k G)$,

$$
0=\left(b-\operatorname{Br}_{P}(b)\right)^{p^{n}}=b^{p^{n}}-\operatorname{Br}_{P}(b)^{p^{n}}=b-\operatorname{Br}_{P}(b)
$$

for $n$ large enough. In particular, $b \in k C_{G}(P)$.

For each block $b \in Z(k G)$, since $b \in k C_{G}(P)$ by (a), $\operatorname{Br}_{P}(b)=b \neq 0$, and so (b) follows from Lemma 5.4 (a).

The following is one immediate consequence of Lemma 5.6(a):

Corollary 5.7. For any p-subgroup $P \leq G$, the groups $P C_{G}(P)$ and $C_{G}(P)$ have the same blocks. 
5.3. Brauer pairs and fusion systems of blocks. The following definitions, which define fusion for a block in analogy with fusion in a group, are due to Alperin and Broué $[\mathrm{AB}$.

Definition 5.8. (a) A Brauer pair in $G$ is a pair $\left(P, b_{P}\right)$, where $P$ is a $p$-subgroup of $G$ and $b_{P}$ is a block of $k P C_{G}(P)$ (equivalently, a block of $k C_{G}(P)$ ).

(b) If $\left(Q, b_{Q}\right)$ and $\left(P, b_{P}\right)$ are two Brauer pairs in $G$, then $\left(Q, b_{Q}\right) \unlhd\left(P, b_{P}\right)$ if

$$
Q \unlhd P, \quad b_{Q} \in Z\left(k C_{G}(Q)\right)^{P} \quad \text { and } \quad b_{P} \operatorname{Br}_{P}\left(b_{Q}\right)=b_{P} .
$$

(c) If $\left(Q, b_{Q}\right)$ and $\left(P, b_{P}\right)$ are two Brauer pairs in $G$, then $\left(Q, b_{Q}\right) \leq\left(P, b_{P}\right)$ if there is a sequence of normal inclusions $\left(Q, b_{Q}\right) \unlhd\left(Q_{1}, b_{1}\right) \unlhd \cdots \unlhd\left(Q_{k}, b_{k}\right) \unlhd$ $\left(P, b_{P}\right)$.

For any Brauer pair $\left(P, b_{P}\right)$, set

$$
\begin{aligned}
& N_{G}\left(P, b_{P}\right)=\left\{\left.x \in N_{G}(P)\right|^{x}\left(P, b_{P}\right)=\left(P, b_{P}\right)\right\}, \\
& C_{G}\left(P, b_{P}\right)=C_{G}(P) \cap N_{G}\left(P, b_{P}\right) .
\end{aligned}
$$

Proposition 5.9 ([AB, (2.9)], Alp2, Theorem 15.1]). Assume that $P \unlhd G$ is a normal p-subgroup, and let $(P, b)$ be any Brauer pair. Let $B \in Z\left(k C_{G}(P)\right)$ be the sum of the distinct idempotents in the $G$-orbit of $b$. Then the following hold.

(a) $B$ is a block of $k G, B=b^{G}$, and is the unique block of $k G$ such that $b \mid B$.

(b) If $D$ is a defect group of $b$ in $N_{G}(P, b)$, then $D$ is also a defect group of $B$ in $G$ and $D \cap P C_{G}(P)$ is a defect group of $b$ in $P C_{G}(P)$.

Most of the results listed so far hold without the assumption that $k$ be algebraically closed. But this assumption is necessary in the next proposition.

Proposition 5.10. Let $P \unlhd G$ be a normal p-subgroup, and let $(P, b)$ be a Brauer pair such that $G=N_{G}(P, b)$ (equivalently, $b \in C_{k C_{G}(P)}(G)$ ). Let $D$ be a defect group for $b$ in $G$. Then

$$
D C_{G}(P) / C_{G}(P) \in \operatorname{Syl}_{p}\left(G / C_{G}(P)\right) .
$$

Proof. Set $H=P C_{G}(P)$ and $\widehat{H}=H \times H$ for short, and let $\widehat{G} \leq G \times G$ be the subgroup of pairs $(g, h)$ such that $g \equiv h(\bmod H)$. Then $b \cdot k H$ is a $k \widehat{G}$-module which is indecomposable as a $k \widehat{H}$-module, and has vertex $\Delta D$. So by Alp2, Lemma 9.8], $\Delta D \cdot \widehat{H} / \widehat{H}$ is a Sylow $p$-subgroup of $\widehat{G} / \widehat{H}$, and hence $D H / H \in \operatorname{Syl}_{p}(G / H)$.

Proposition 5.11 ( $\mathrm{AB}$, Lemmas 3.9 and 3.10, Corollary 3.12]). Let $\left(P, b_{P}\right)$ be a Brauer pair in $G$, and let $\mathcal{B}_{\left(P, b_{P}\right)}^{G}$ be the set of all Brauer pairs $\left(Q, b_{Q}\right)$ in $G$ such that $\left(P, b_{P}\right) \unlhd\left(Q, b_{Q}\right)$.

(a) $A$ pair $\left(R, b_{R}\right) \in \mathcal{B}_{\left(P, b_{P}\right)}^{G}$ is maximal in $\mathcal{B}_{\left(P, b_{P}\right)}^{G}$ if and only if $R$ is a defect group of $b_{P}$ in $N_{G}\left(P, b_{P}\right)$.

(b) The group $N_{G}\left(P, b_{P}\right)$ acts transitively by conjugation on the set of maximal pairs in $\mathcal{B}_{\left(P, b_{P}\right)}^{G}$.

The next proposition is a reformulation of Proposition 5.11

Proposition 5.12. Let $\left(P, b_{P}\right)$ be a Brauer pair for $G$, and fix $H$ such that $P C_{G}(P) \leq H \leq N_{G}\left(P, b_{P}\right)$. Let $\mathcal{B}_{\left(P, b_{P}\right)}^{H}$ be the set of all Brauer pairs $\left(Q, b^{\prime}\right)$ such that $\left(P, b_{P}\right) \unlhd\left(Q, b^{\prime}\right)$ and $Q \leq H$. Then the maximal elements of $\mathcal{B}_{\left(P, b_{P}\right)}^{H}$ are exactly those of the form $(D, b)$, where $D$ is a defect group of $b_{P}$ in $H$, and where 
$b$ is a primitive central idempotent such that $b \mid \mathrm{Br}_{D}\left(b_{P}\right)$. In particular, any two maximal elements of $\mathcal{B}_{\left(P, b_{P}\right)}^{H}$ are $H$-conjugate.

Proof. It suffices to prove this when $G=H$, in which case it follows from Proposition 5.11. Note, since $H \geq P C_{G}(P)$, that $\left(P, b_{P}\right)$ is also a Brauer pair in $H$, and also that (in the notation of the proposition) $\mathcal{B}_{\left(P, b_{P}\right)}^{H}$ contains all pairs $\left(Q, b_{Q}\right) \in \mathcal{B}_{\left(P, b_{P}\right)}^{G}$ such that $Q \leq H$.

Note that for any block $b$ of $G$, the pair $(1, b)$ is a Brauer pair.

Definition 5.13. Let $b$ be a block of $k G$. A Brauer pair $\left(P, b_{P}\right)$ is a $b$-subgroup (or associated to $b$ ) if $b_{P} \mid \operatorname{Br}_{P}(b)$; equivalently, $(1, b) \unlhd\left(P, b_{P}\right)$.

The following two propositions help to explain why it is reasonable to refer to subgroups and inclusions here.

Proposition 5.14. If $b$ is a block of $G,\left(P, b_{P}\right)$ is a b-subgroup, and $\left(Q, b_{Q}\right) \leq$ $\left(P, b_{P}\right)$, then $\left(Q, b_{Q}\right)$ is also a b-subgroup.

Proof. It suffices to show this when $\left(Q, b_{Q}\right) \unlhd\left(P, b_{P}\right)$. In that case,

$$
b_{P} \mid \operatorname{Br}_{P / Q}\left(b_{Q}\right) \quad \text { and } \quad b_{P} \mid \operatorname{Br}_{P}(b)=\operatorname{Br}_{P / Q}\left(\operatorname{Br}_{Q}(b)\right) \text {, }
$$

so $b_{Q} \cdot \operatorname{Br}_{Q}(b) \neq 0$, and $b_{Q} \mid \operatorname{Br}_{Q}(b)$ since $b_{Q}$ is primitive.

Proposition 5.15 ( $\mathrm{AB}$, Theorem 3.4], Alp2, Theorem 16.3]). For each Brauer pair $\left(P, b_{P}\right)$ for $G$ and each $Q \leq P$, there is a unique block $b_{Q}$ of $C_{G}(Q)$ such that $\left(Q, b_{Q}\right) \leq\left(P, b_{P}\right)$. Furthermore, $\left(Q, b_{Q}\right) \unlhd\left(P, b_{P}\right)$ if and only if $Q \unlhd P$.

As an example, consider the principal block $b_{0}=b_{0}(G)$ of $k G$. The $b_{0}$-subgroups are exactly the Brauer pairs $\left(P, b_{0}\left(P C_{G}(P)\right)\right)$ for all $p$-subgroups $P \leq G$. To see this, note first that since $b_{0}$ has augmentation (sum of coefficients) $\epsilon\left(b_{0}\right)=1$, so does $\operatorname{Br}_{P}\left(b_{0}\right)$ for each $p$-subgroup $P$. Hence $\operatorname{Br}_{P}\left(b_{0}\right) \cdot k P C_{G}(P)$ contains the trivial module $k$ (the idempotent acts on $k$ via the identity), so the principal block $b_{0}\left(P C_{G}(P)\right)$ is contained in $\operatorname{Br}_{P}\left(b_{0}\right)$, and hence $\left(1, b_{0}\right) \unlhd\left(P, b_{P}\right)$. Proposition 5.12 (all maximal $b_{0}$-subgroups are conjugate) now implies that these are the only $b_{0^{-}}$ subgroups when $P \in \operatorname{Syl}_{p}(G)$, and then Proposition 5.15 shows that these are the only $b_{0}$-subgroups for arbitrary $P$.

Definition 5.16. Fix a block $b$ of $k G$ and a defect group $D$ of $b$, and let $B_{D}$ be the block of $k N_{G}(D)$ of defect group $D$ such that $\left(B_{D}\right)^{G}=b$ (Brauer's first main theorem). Let $b_{D}$ be a block of $k D C_{G}(D)$ which is a primitive component of $B_{D}$. For each $P \leq D$, let $b_{P}$ be the unique block of $k C_{G}(P)$ such that $\left(P, b_{P}\right) \leq\left(D, b_{D}\right)$. Let $\mathcal{F}_{D}(b)=\mathcal{F}_{\left(D, b_{D}\right)}(G, b)$ be the fusion system over $D$ defined by setting

$$
\operatorname{Hom}_{\mathcal{F}_{D}(b)}(P, Q)=\left\{c_{x} \in \operatorname{Hom}_{G}(P, Q) \mid x \in G,{ }^{x}\left(P, b_{P}\right) \leq\left(Q, b_{Q}\right)\right\} .
$$

We first check that this is a fusion system.

Lemma 5.17. For each $P, Q \leq D, \operatorname{Hom}_{D}(P, Q) \subseteq \operatorname{Hom}_{\mathcal{F}_{D}(b)}(P, Q)$.

Proof. If $x \in D$ and ${ }^{x} P \leq Q$, then ${ }^{x}\left(P, b_{P}\right) \leq\left(Q, b_{Q}\right)$ by Proposition 5.15] and since both are contained in $(D, b)={ }^{x}(D, b)$. Hence $c_{x} \in \operatorname{Hom}_{\mathcal{F}_{D}(b)}(P, Q)$.

Thus $\mathcal{F}_{D}(b)$ is a fusion system over $D$. It remains to show that it is saturated.

Lemma 5.18. If $P \leq D$ is such that $N_{D}(P)$ is a defect group of $b_{P}$ in $N_{G}\left(P, b_{P}\right)$, then $P$ is fully automized and receptive in $\mathcal{F}_{D}(b)$. 
Proof. By Proposition 5.10 (applied with $N_{G}\left(P, b_{P}\right)$ in the role of $G$ ), and since $N_{D}(P)$ is a defect group of $b_{P}$,

$$
\operatorname{Aut}_{D}(P) \cong N_{D}(P) C_{G}\left(P, b_{P}\right) / C_{G}\left(P, b_{P}\right) \in \operatorname{Syl}_{p}\left(N_{G}\left(P, b_{P}\right) / C_{G}\left(P, b_{P}\right)\right)
$$

where $N_{G}\left(P, b_{P}\right) / C_{G}\left(P, b_{P}\right) \cong \operatorname{Aut}_{\mathcal{F}_{D}(b)}(P)$. So $P$ is fully automized in $\mathcal{F}_{D}(b)$.

It remains to prove that $P$ is receptive. Fix a subgroup $Q \leq D$ and a morphism $c_{x} \in \operatorname{Hom}_{\mathcal{F}_{D}(b)}(Q, P)$, and set

$$
N_{x}=\left\{g \in N_{D}(Q) \mid c_{x g x^{-1}} \in \operatorname{Aut}_{D}(P)\right\}=\left\{g \in N_{D}(Q) \mid{ }^{x} g \in N_{D}(P) \cdot C_{G}\left(P, b_{P}\right)\right\} .
$$

The Brauer pair $\left(N_{x}, b_{N_{x}}\right)$ contains $\left(Q, b_{Q}\right)$ as a normal subpair, so the pair ${ }^{x}\left(N_{x}, b_{N_{x}}\right)$ contains $\left(P, b_{P}\right)$ as a normal subpair. By Proposition 5.12, and since $N_{D}(P)$ is a defect group of $b_{P}$ in $N_{G}\left(P, b_{P}\right),\left(N_{D}(P), b_{N_{D}(P)}\right)$ is maximal among Brauer pairs in $N_{G}\left(P, b_{P}\right)$ which contain $\left(P, b_{P}\right)$ as a normal subpair, and hence also among such pairs in $N_{D}(P) C_{G}\left(P, b_{P}\right)$. So by the same proposition, there is $y \in C_{G}\left(P, b_{P}\right)$ such that ${ }^{y x}\left(N_{x}\right) \leq N_{D}(P)$. In other words, $c_{y x} \in \operatorname{Hom}_{\mathcal{F}_{D}(b)}\left(N_{x}, N_{D}(P)\right)$, and thus $P$ is receptive.

We are now ready to prove the following theorem, which is due to Puig Pg5. Theorem 3.7] and which is a special case of [AKO, Theorem IV.3.2].

Theorem 5.19. For each block $b$ in $k G$ and each maximal Brauer pair $\left(D, b_{D}\right)$ associated to $b$, the fusion system $\mathcal{F}_{D}(b)=\mathcal{F}_{\left(D, b_{D}\right)}(G, b)$ is saturated.

Proof. Fix a subgroup $P \leq D$, and let $b_{P}$ be such that $\left(P, b_{P}\right) \leq\left(D, b_{D}\right)$. We must show that $P$ is $\mathcal{F}_{D}(b)$-conjugate to a subgroup of $D$ which is fully automized and receptive in $\mathcal{F}_{D}(b)$.

Let $\left(D_{0}, b^{\prime}\right)$ be maximal among Brauer pairs in $G$ such that $\left(P, b_{P}\right) \unlhd\left(D_{0}, b^{\prime}\right)$. Then $(1, b) \unlhd\left(D_{0}, b^{\prime}\right)$ by Proposition 5.15, so by Proposition 5.11, there is $x \in G$ such that $\left(D_{0}, b^{\prime}\right) \leq x^{-1}\left(D, b_{D}\right)$. Hence $\left(D, b_{D}\right) \geq{ }^{x}\left(D_{0}, b^{\prime}\right)$.

Thus ${ }^{x}\left(P, b_{P}\right) \unlhd{ }^{x}\left(D_{0}, b^{\prime}\right) \leq\left(D, b_{D}\right)$. Set $Q={ }^{x} P$; then ${ }^{x}\left(P, b_{P}\right)=\left(Q, b_{Q}\right)$ by Proposition [5.15] so $Q$ is $\mathcal{F}_{D}(b)$-conjugate to $P$. Also, ${ }^{x}\left(D_{0}, b^{\prime}\right)$ is maximal among Brauer pairs such that $\left(Q, b_{Q}\right) \unlhd{ }^{x}\left(D_{0}, b^{\prime}\right)$, and since $\left(N_{D}(Q), b_{N_{D}(Q)}\right)$ is another such pair, we have ${ }^{x} D_{0}=N_{D}(Q)$. Thus $N_{D}(Q)$ is a defect group of $b_{Q}$ in $N_{G}\left(Q, b_{Q}\right)$ by Proposition 5.12, and hence $Q$ is fully automized and receptive in $\mathcal{F}_{D}(b)$ by Lemma 5.18

In fact, the above argument can be modified slightly to show that in that situation, a subgroup $P \leq D$ is fully normalized in $\mathcal{F}_{D}(b)$ (fully centralized in $\mathcal{F}_{D}(b)$ ) if and only if $N_{D}(P)$ is a defect group of $b_{P}$ in $N_{G}\left(P, b_{P}\right)\left(C_{D}(P)\right.$ is a defect group of $b_{P}$ in $\left.C_{G}\left(P, b_{P}\right)\right)$.

When $b_{0}$ is the principal block of $k G$, we have already seen that the $b_{0}$-subgroups are the Brauer pairs $\left(P, b_{P}\right)$, where $P \leq G$ is an arbitrary $p$-subgroup, and $b_{P}$ is the principal block of $k P C_{G}(P)$. Hence $N_{b_{0}}(P, Q)=N_{G}(P, Q)$ for all pairs of $p$ subgroups $P, Q \leq G, \operatorname{Hom}_{b_{0}}(P, Q)=\operatorname{Hom}_{G}(P, Q)$, and the fusion system of $b_{0}$ is just the fusion system of the group $G$.

5.4. Applications to the representation theory of blocks. We now give some examples of how the fusion system of a block influences, or even helps to determine, its representation theory.

As a first example, the definition in $\overline{\mathrm{BP}}$ of a nilpotent block is really a condition on the fusion system of the block: a block $b$ in $k G$ with defect group $D$ is nilpotent if 
$\mathcal{F}_{D}(b)=\mathcal{F}_{D}(D)$. The main results in $\mathrm{BP}$ and in $\mathrm{Pg} 2$ describe how this property restricts the representation type of the algebra $b \cdot k G$. For example, whenever $b$ is nilpotent, there is exactly one irreducible Brauer character of $G$ in $b$, and thus exactly one simple $b \cdot k G$-module $[\mathrm{BP}$, Theorem $1.2(3)]$. Also, for each Brauer pair $(P, e)$ associated to $b, e$ is a nilpotent block of $k C_{G}(P)$.

The next proposition describes some other situations where the fusion system of a block determines the number of its irreducible representations. Just by looking at the original formulations of these results, one sees the advantage of expressing them in terms of the fusion system of the block. Recall the definition of $\mathcal{F}$-radical (Definition 1.13).

Proposition 5.20. Let $b$ be a block in $k G$ with defect group $D$, and let $\operatorname{Irr}(b \cdot k G)$ be the set of irreducible $b \cdot k G$-modules up to isomorphism (equivalently, the set of Brauer characters of $G$ in the block b). Set $\mathcal{F}=\mathcal{F}_{D}(b)$.

(i) If $D$ is cyclic, $D \cong C_{2}^{2}$, or $D \cong Q_{8}$, then $|\operatorname{Irr}(b \cdot k G)|=\left|\operatorname{Out}_{\mathcal{F}}(D)\right|$.

(ii) Assume that $p=2$ and that $D$ is dihedral of order at least 8 or semidihedral or quaternion of order at least 16 . Let $m$ be the number of $S$-conjugacy classes of $\mathcal{F}$-radical subgroups in $D(m=|I|$ in the notation of Example 1.17). Then $|\operatorname{Irr}(b \cdot k G)|=m+1$.

Proof. The cyclic case is shown in [Da1, Theorem 1], the case $D \cong C_{2}^{2}$ in [Br1, $\left.\S \mathrm{VII}\right]$, the other dihedral cases in [Br2, Theorem 2], and the semidihedral and quaternion cases (including $Q_{8}$ ) in [Ols, p. 231].

The number of ordinary (complex) characters associated with $b$ is also determined in each of the above cases, in terms of the fusion system $\mathcal{F}_{D}(b)$, in the above references.

The number of simple $b \cdot k G$-modules does not, in general, depend only on the fusion system of the block $b$ (see, e.g., AKO, Example IV.5.43]). However, Alperin's weight conjecture Alp3 can be interpreted as a formula for $|\operatorname{Irr}(b \cdot k G)|$ in terms of the fusion system of $b$ together with certain cohomology classes constructed by Külshammer and Puig $[\mathrm{KP}$.

More concretely, fix a block $b$ in $k G$, let $\left(D, b_{D}\right)$ be a maximal Brauer pair associated to $b$, and set $\mathcal{F}=\mathcal{F}_{\left(D, b_{D}\right)}(G, b)$. Let $Q \leq D$ be an $\mathcal{F}$-centric subgroup, and let $b_{Q}$ be such that $\left(Q, b_{Q}\right) \leq\left(D, b_{D}\right)$. Set $C_{G}^{\prime}(Q)=Q C_{G}(Q) / Q \cong C_{G}(Q) / Z(Q)$, a group of order prime to $p$, and let $\overline{b_{Q}}$ be the primitive idempotent in $Z\left(k C_{G}^{\prime}(Q)\right)$ induced by $b_{Q} \in Z\left(k Q C_{G}(Q)\right)$. Set $A=\overline{b_{Q}} k C_{G}^{\prime}(Q)$, a simple $k$-algebra and hence a matrix algebra over $k$. Consider the following diagram of groups and homomorphisms.

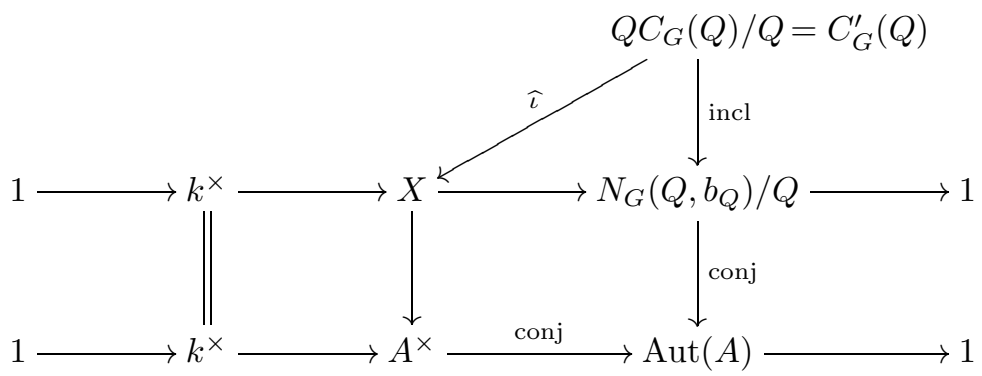


Here, $X$ is the pullback of $A^{\times}$and $N_{G}\left(Q, b_{Q}\right) / Q$ over $\operatorname{Aut}(A)$ :

$$
X=\left\{(g, x) \in N_{G}\left(Q, b_{Q}\right) / Q \times A^{\times} \mid c_{g}=c_{x} \in \operatorname{Aut}(A)\right\} .
$$

Since $\operatorname{Aut}(A)=\operatorname{Inn}(A)$ by the Skolem-Noether theorem (cf. Be1, Proposition 1.3.6]), the bottom row is exact, and hence both rows are central extensions of groups. For $h \in C_{G}^{\prime}(Q)$, we set $\widehat{\iota}(h)=\left(h, h \cdot \overline{b_{Q}}\right)$. It is straightforward to check that $\widehat{\iota}\left(C_{G}^{\prime}(Q)\right) \unlhd X$, and hence that the quotient group $X / \widehat{\iota}\left(C_{G}^{\prime}(Q)\right)$ is a central extension of $N_{G}\left(Q, b_{Q}\right) / Q C_{G}(Q) \cong \operatorname{Out}_{\mathcal{F}}(Q)$ by $k^{\times}$. The Külshammer-Puig class for $Q, \alpha_{Q} \in H^{2}\left(\operatorname{Out}_{\mathcal{F}}(Q) ; k^{\times}\right)$, is the cohomology invariant of this central extension.

For each class $\alpha_{Q}$, represented by a 2-cocycle $\widetilde{\alpha}_{Q}: \operatorname{Out}_{\mathcal{F}}(Q) \times \operatorname{Out}_{\mathcal{F}}(Q) \longrightarrow k^{\times}$, the twisted group algebra $k_{\alpha_{Q}} \operatorname{Out}_{\mathcal{F}}(Q)$ is defined to be the $k$-vector space with basis $\operatorname{Out}_{\mathcal{F}}(Q)$, and with multiplication defined by setting

$$
(\alpha) \cdot(\beta)=\widetilde{\alpha}_{Q}(\beta, \alpha) \cdot(\alpha \beta) .
$$

The assumption that $\widetilde{\alpha}_{Q}$ is a cocycle ensures that this multiplication is associative, and the resulting algebra depends (up to isomorphism) only on the cohomology class $\alpha_{Q}$.

For a finite dimensional $k$-algebra $A$, let $z(A)$ be the number (up to isomorphism) of simple $A$-modules which are projective. In these terms, Alperin's weight conjecture can be reformulated as follows.

Conjecture 5.21 ([ $\mathrm{Ke} 2$, Proposition 5.4]). Let $b$ be a block in $k G$ with defect group $D$, and let $\operatorname{Irr}(b \cdot k G)$ be the set of irreducible $b \cdot k G$-modules up to isomorphism. Set $\mathcal{F}=\mathcal{F}_{D}(b)$. Let $\mathcal{X}$ be a set of $\mathcal{F}$-conjugacy class representatives for the $\mathcal{F}$-centric $\mathcal{F}$-radical subgroups of $D$. For each $Q \in \mathcal{X}$, let $\alpha_{Q} \in H^{2}\left(\operatorname{Out}_{\mathcal{F}}(Q) ; k^{\times}\right)$be its Külshammer-Puig class, and let $k_{\alpha_{Q}} \operatorname{Out}_{\mathcal{F}}(Q)$ be the algebra induced by $\alpha_{Q}$. Then

$$
|\operatorname{Irr}(b \cdot k G)|=\sum_{Q \in \mathcal{X}} z\left(k_{\alpha_{Q}} \operatorname{Out}_{\mathcal{F}}(Q)\right) .
$$

As one simple example, when the block $b$ is nilpotent (when $\mathcal{F}=\mathcal{F}_{D}(D)$ ), $D$ is the only subgroup which is $\mathcal{F}$-centric and $\mathcal{F}$-radical, and $\operatorname{Out}_{\mathcal{F}}(D)=1$. Thus the theorem of Broué and Puig that $|\operatorname{Irr}(b \cdot k G)|=1$ is a special case of this conjecture.

As a second example, in the situation of Proposition 5.20(i,ii), the KülshammerPuig classes $\alpha_{Q}$ all vanish, since the groups $H^{2}\left(\operatorname{Out}_{\mathcal{F}}(Q) ; k^{\times}\right)$themselves all vanish (see AKO, Proposition IV.5.33]). So the above formula involves only ordinary group rings. Proposition 5.20 thus follows as a special case of the conjecture, upon checking that $z(k G)=|G|$ when $G$ is abelian of order prime to $p$, and (when $\operatorname{char}(k)=2$ ) that $z\left(k C_{2}\right)=0$ and $z\left(k \Sigma_{3}\right)=1$.

In an attempt to give yet another interpretation to Alperin's conjecture, Linckelmann conjectured that the Külshammer-Puig classes can all be "glued" together to give one single cohomology class.

Conjecture 5.22 (Gluing conjecture [Li1, Conjecture 4.2]). Let $b$ be a block in $k G$ with defect group $D$, and set $\mathcal{F}=\mathcal{F}_{D}(b)$. Then there is an element $\alpha \in$ $H^{2}\left(\mathcal{O}\left(\mathcal{F}^{c}\right) ; k^{\times}\right)$whose restriction to $\operatorname{Out}_{\mathcal{F}}(Q)$, for each $\mathcal{F}$-centric subgroup $Q$, is the Külshammer-Puig class $\alpha_{Q}$.

Linckelmann has also constructed [Li2, Theorems 1.1-1.2] an exact sequence involving the map $H^{2}\left(\mathcal{O}\left(\mathcal{F}^{c}\right) ; k^{\times}\right) \longrightarrow \lim _{Q} H^{2}\left(\operatorname{Out}_{\mathcal{F}}(Q) ; k^{\times}\right)$, thus helping to describe its kernel and cokernel. It is known, through an example constructed by Park [Pa], that this homomorphism need not be injective, and hence that the class 
$\alpha$ in Conjecture 5.22 need not be unique. Other results about the gluing conjecture, including some special cases, were proven by Libman in Lb1, Lb2].

The motivation for the gluing conjecture arises through a categorical version of twisted group algebras. In general, when $\mathcal{C}$ is a small category and $R$ is a commutative ring, $R \mathcal{C}$ denotes the (ordinary) group algebra of $\mathcal{C}$ : the free $R$-module with basis the set $[\varphi]$ for $\varphi \in \operatorname{Mor}(\mathcal{C})$, and where $[\varphi] \cdot[\psi]=[\varphi \psi]$ if $\varphi$ and $\psi$ are composable and $[\varphi] \cdot[\psi]=0$ otherwise. We want to define a twisted version of this: an algebra twisted by a cohomology class $\alpha \in H^{2}\left(\mathcal{C} ; R^{\times}\right)$. We refer to AKO, $\S$ III.5.1] for a very brief introduction to the cohomology (equivalently, higher limits) of a category with coefficients in a functor. Here, we consider only coefficients in a constant functor: a functor which sends each object to a fixed abelian group $A$ and sends all morphisms to $\operatorname{Id}_{A}$.

For each $n \geq 2$, let $T_{n}(\operatorname{Mor}(\mathcal{C}))$ be the set of $n$-tuples of composable morphisms; more precisely, the set of all $\left(\varphi_{1}, \ldots, \varphi_{n}\right) \in \operatorname{Mor}(\mathcal{C})^{n}$ such that $\varphi_{n} \circ \cdots \circ \varphi_{1}$ is defined (composing from right to left). A cohomology class $\alpha \in H^{2}\left(\mathcal{C} ; R^{\times}\right)$(i.e., the cohomology of the constant functor on $\mathcal{C}$ which sends each object to $R^{\times}$) is represented by a 2-cocycle $t: T_{2}(\operatorname{Mor}(\mathcal{C})) \longrightarrow R^{\times}$; i.e., a function such that $t(\psi, \chi) \cdot t(\varphi, \chi \psi)=t(\varphi, \psi) \cdot t(\psi \varphi, \chi)$ for each $(\varphi, \psi, \chi) \in T_{3}(\operatorname{Mor}(\mathcal{C}))$. The twisted category algebra $R_{\alpha} \mathcal{C}$ is the free $R$-module, again with basis the $[\varphi]$ for $\varphi \in \operatorname{Mor}(\mathcal{C})$, where

$$
[\psi] \cdot[\varphi]= \begin{cases}t(\varphi, \psi) \cdot[\psi \varphi] & \text { if }(\varphi, \psi) \in T_{2}(\operatorname{Mor}(\mathcal{C})), \\ 0 & \text { otherwise. }\end{cases}
$$

By [Li1, Theorem 4.3], if the class $\alpha$ exists as described in the gluing conjecture, then Alperin's conjecture is equivalent to the following: in the situation of Conjecture [5.21. $|\operatorname{Irr}(b \cdot k G)|$ is equal to the number of isomorphism classes of simple $k_{\alpha} \mathcal{O}\left(\mathcal{F}^{c}\right)$-modules $M$ for which $M$ has multiplicity 1 in the composition series of its projective cover.

\section{Generalizations}

One can, of course, define a fusion system over any group (or any " $p$-group" in some very general sense), and look for "saturation" conditions analogous to those in Definition 1.2. However, what one really is interested in are those which occur naturally in some sense: for example, by modelling local structures or classifying spaces on certain types of groups. In this section, we first discuss fusion systems over "discrete p-toral groups" (modeling compact Lie groups), which seem to be the most successful generalizations so far, and then look at two other cases which have been considered.

6.1. Fusion systems over discrete $p$-toral groups. Set $\mathbb{Z} / p^{\infty}=\mathbb{Z}[1 / p] / \mathbb{Z}$; one can regard this as the union of the cyclic groups $\mathbb{Z} / p^{m}$ under the obvious inclusions $\mathbb{Z} / p^{m}<\mathbb{Z} / p^{m+1}$. A discrete $p$-torus is a group isomorphic to $\left(\mathbb{Z} / p^{\infty}\right)^{k}$ for some $k \geq 0$. A discrete $p$-toral group is a group which contains a discrete $p$-torus as a normal subgroup of $p$-power index. The class of discrete $p$-toral groups is clearly closed under taking subgroups, quotient groups, and extensions.

Saturated fusion systems over discrete $p$-toral groups, and linking systems associated to them, are defined almost in the same way as those over finite $p$-groups. The main difference is that an extra "continuity" condition on the set of morphisms in the fusion system is needed. By analogy with the finite case, a classifying space 
of a saturated fusion system over a discrete $p$-toral group $\mathcal{F}$ is the $p$-completed realization $|\mathcal{L}|_{p}^{\wedge}$ of some associated linking system $\mathcal{L}$. We refer to BLO3. Definitions 2.2 and 4.1] for precise definitions, and to [BLO3] and [BLO5] for some of the properties of fusion and linking systems in this setting. More recently, Levi and Libman LL] have proven the existence and uniqueness of linking systems (and hence classifying spaces) associated to saturated fusion systems over a discrete $p$-toral group.

If $G$ is a compact Lie group with maximal torus $T$, and $T_{\infty} \leq T$ denotes the subgroup of elements of $p$-power order, then $T_{\infty}$ is a discrete $p$-torus. Its extension by a Sylow $p$-subgroup of $N_{G}(T) / T$ is a maximal discrete $p$-toral subgroup of $G$, and is $G$-conjugate to all other maximal discrete $p$-toral subgroups. Thus $G$ contains maximal discrete $p$-toral subgroups which are unique up to conjugacy, and which can be regarded as its "Sylow $p$-subgroups". Starting with this observation, one can define a saturated fusion system $\mathcal{F}_{S}(G)$ just as in the finite case, and (with a lot more work than in the finite case) a centric linking system $\mathcal{L}_{S}^{c}(G)$. Also, $\left|\mathcal{L}_{S}^{c}(G)\right|_{p}^{\wedge} \simeq B G_{p}^{\wedge}$ for all such $G$. We refer to [BLO3, $\left.\S 9\right]$ for all of these results.

In addition to compact Lie groups, saturated fusion systems over discrete $p$-toral groups and their classifying spaces can also be used to model $p$-compact groups, and $p$-completed classifying spaces of torsion linear groups (torsion subgroups of $G L_{n}(K)$ where $K$ is a field and $\left.\operatorname{char}(K) \neq p\right)$. See [BLO3, Theorem 8.10 and $\S 10$ ] for details.

Most recently, Gonzalez $\mathrm{Gz}$ has shown that fusion and linking systems over discrete $p$-toral groups can be approximated, in a way which he makes precise, by fusion and linking systems over finite $p$-groups. He was then able to use this to prove certain homotopy properties of the spaces $|\mathcal{L}|_{p}^{\wedge}($ or $|\mathcal{L}|)$ which had not been shown in [BLO3], and which turned out to be much more difficult than in the finite case. For example, he showed that in such cases, $H^{*}\left(|\mathcal{L}| ; \mathbb{F}_{p}\right)$ is isomorphic to the inverse limit (taken for $P$ in $\mathcal{F}$ ) of cohomology groups $H^{*}\left(P ; \mathbb{F}_{p}\right)$, just as in the finite case. As a result, all of the properties of classifying spaces of saturated fusion systems over finite $p$-groups listed in Table 2 are now known in the discrete $p$-toral case; these are shown either in BLO3, or in $\mathrm{Gz}$.

As one example, when $\mathbb{G}$ is a connected, reductive group scheme over $\mathbb{Z}$ (e.g., $\mathbb{G}=G L_{n}, P S L_{n}$, or $\left.S p_{2 n}\right)$, and $p$ and $q$ are distinct primes, then the $p$-fusion system of $\mathbb{G}\left(\overline{\mathbb{F}}_{q}\right)$ (over a discrete $p$-toral group) can be approximated by the $p$ fusion systems of the finite groups $\mathbb{G}\left(q^{n}\right)$ for $n \geq 1$. Gonzalez constructed such an approximation in the general case by taking fixed point subsystems under an appropriate sequence of "unstable Adams operations" constructed by Junod, Levi, and Libman [JLL].

As another example, the Solomon fusion systems $\operatorname{Sol}(q)$ (see section 1.12) can be used to approximate the fusion system of the 2-compact group $B D I(4)$ constructed by Dwyer and Wilkerson. This had been predicted by Benson Be2 (and was a large part of his motivation for predicting that one can associate classifying spaces to fusion systems) and was proven by Levi and Oliver in [LO, Theorem 4.5].

6.2. Fusion systems over pro- $p$-groups. In [SSy, the authors study pro-fusion systems over pro- $p$-groups; i.e., inverse limits of fusion systems over (finite) $p$ groups. These are regarded as fusion systems in the sense of Definition 1.1, but with $S$ assumed to be a pro- $p$-group instead of a finite $p$-group [SSy, Lemmas 2.9 and 2.12]. However, the objects in a pro-fusion system $\mathcal{F}$ over $S$ are the closed subgroups of $S$, and the condition that $\mathcal{F}$ be an inverse limit of fusion systems over 
finite quotients imposes additional conditions on the morphisms. For example, if $P$ is an open subgroup of index $p^{n}$ in $S$, then each $Q \in P^{\mathcal{F}}$ must also be open of index $p^{n}$ in $S$.

Fully automized and receptive subgroups, and through that saturation of a profusion system, are defined by analogy with Definition 1.2 in the finite case SSy, Definitions 2.14-2.16]. A pro-p-group is countably based if it is isomorphic to an inverse limit of a system of finite $p$-groups indexed by $\mathbb{N}$; equivalently, if it has countably many open subgroups. By [SSy, Theorem 5.2], a pro-saturated fusion system (i.e., inverse limit of saturated fusion systems) over a countably based prop-group is saturated.

Finally, in SSy, §6-7], the authors prove a version of Alperin's fusion theorem in this situation. The key difference with the usual theorem (see Theorems 1.14 and 1.19) is that in a fusion system over a pro- $p$-group, a morphism might be the composite of a convergent, infinite sequence of restrictions of $\mathcal{F}$-automorphisms.

All of this theory applies to fusion systems for pro-finite groups. In particular, this result provides a version of Alperin's fusion theorem for pro-finite groups.

6.3. Fusion systems over $p$-unipotent groups of finite Morley rank. In ABC, $\S$ X.4.1], the authors speculate about working out a theory of saturated fusion systems over $p$-unipotent groups of finite Morley rank, especially when $p=2$. Just as for fusion systems over pro-p-groups, this would require restrictions on the subgroups and morphisms in the category: not all subgroups would be objects, and not all injective homomorphisms between objects are allowed. They feel that this could be useful in developing further the theory of groups of finite Morley rank; e.g., "the interaction between the direct study of fusion and the amalgam method".

\section{OpEn QUESTIONS AND PROBLEMS}

Question 7.1. Let $p$ be a prime. Define an $N_{p}$-system to be a saturated $p$-fusion system in which all local subsystems are Puig solvable.

Suppose $\mathcal{F}$ is an $N_{p}$-system on $S$. One possibility is that $O_{p}(\mathcal{F}) \neq 1$. In this case $\mathcal{F}$ is local and hence Puig solvable by hypothesis, so $\mathcal{F}$ is constrained with $p$ solvable model. If $p>3$, then an extended exercise using Alperin's fusion theorem and Glauberman's ZJ Theorem [G12] shows that $O_{p}(\mathcal{F}) \neq 1$. But if $p=2$ or 3 , there are nonsolvable examples.

Indeed the $N_{2}$-systems are the $N$-systems of section 2.8 so such systems are determined in A9.

What if $p=3$ ? Examples of nonsolvable $N_{3}$-systems include the 3 -fusion systems of $L_{3}(3)$, Aut $\left(L_{3}(3)\right),{ }^{2} F_{4}(2)^{\prime}$, and ${ }^{2} F_{4}(2)$; these are the systems with $S \cong 3^{1+2}$. There are also the 3 -fusion systems of almost simple groups with generalized Fitting subgroup $\mathrm{PSp}_{4}(3)$ and $G_{2}(3)$, and perhaps similar examples for a few more small simple groups. Problem: classify all $N_{3}$-systems.

Question 7.2. Are the Solomon systems the only exotic simple 2-fusion systems? The search for exotic simple 2-fusion systems is reminiscent of the search for sporadic simple groups.

Question 7.3. Let $\mathcal{F}$ be a saturated fusion system all of whose composition factors are realizable. Is $\mathcal{F}$ realizable? This is of particular importance when $p=2$.

Problem 7.4. Given a saturated $p$-fusion system $\mathcal{F}$ and a saturated subsystem $\mathcal{E}$, suitably embedded in $\mathcal{F}$, define the notion of the centralizer $C_{\mathcal{F}}(\mathcal{E})$ and/or the 
normalizer $N_{\mathcal{F}}(\mathcal{E})$, and prove these objects are saturated subsystems of $\mathcal{F}$ with good properties. For example in Definition 1.8 we have the definition of the centralizer and normalizer of a fully normalized subgroup of $S$, while if $\mathcal{E} \unlhd \mathcal{F}$, then $C_{\mathcal{F}}(\mathcal{E})$ is defined in A4.

Problem 7.5. Let $\mathcal{F}$ be a saturated even 2-fusion system on $S$ in which all simple sections of local subsystems are known. Show that, with known exceptions, there exists a fairly naturally defined pair $\mathcal{F}_{1}, \mathcal{F}_{2}$ of local subsystems on $S$ such that $\mathcal{F}=\left\langle\mathcal{F}_{1}, \mathcal{F}_{2}\right\rangle$. When $\mathcal{F}$ is of characteristic 2-type, this is accomplished in Theorem 5 of A5.

Problem 7.6. Let $p$ be an odd prime. Find some system to the simple (or reduced) $p$-fusion systems: some way to at least begin to organize them.

In [05] and [CrOS, simple and reduced fusion systems were (in part) classified over $p$-groups which have a unique abelian subgroup of index $p$. Even in this very small case, most of the examples found turn out to be exotic (at least, when $p>3$ ). These examples illustrate the difficulties when trying to classify simple $p$-fusion systems. They also suggest that almost all reduced or simple fusion systems over a p-group should be exotic.

One way to make this precise would be (for fixed $p$ ) to let $s(n)$ be the number of distinct simple fusion systems over $p$-groups of order at most $p^{n}$, and let $t(n) \leq s(n)$ be the number of them which are exotic. The conjecture would then be that $\lim _{n \rightarrow \infty}(t(n) / s(n))=1$. But there might be other formulations which are easier to work with.

Question 7.7. For an odd prime $p$, are there any saturated $p$-fusion systems which can be proven to be exotic without appealing to the classification of finite simple groups? What tools or criteria could be used to do this, in at least some cases?

Problem 7.8. Find a fusion system which is realizable but not tame. (See section 3.3 for the definition of tame fusion systems.) By Theorems 3.5 and 3.6. all simple fusion systems of known simple groups are tame. Could there be fusion systems which are reduced but not simple, and also are realizable but not tame?

Question 7.9. Let $G$ be a finite 2-perfect group whose Schur multiplier has odd order (equivalently, a finite group such that $H^{i}(G ; \mathbb{Z} / 2)=0$ for $\left.i=1,2\right)$. Set $\mathcal{F}=\mathcal{F}_{S}(G)$ and $\mathcal{L}=\mathcal{L}_{S}^{c}(G)$ for some $S \in \operatorname{Syl}_{2}(G)$. Is the homomorphism $\mu_{\mathcal{L}}: \operatorname{Out}(\mathcal{L}) \longrightarrow \operatorname{Out}(\mathcal{F})$ always injective (see Definition $[3.3)$ ?

Recall that by Theorem 3.8 (b), when $p$ is odd, $\mu_{\mathcal{L}}$ is an isomorphism for each centric linking system $\mathcal{L}$ over a $p$-group. Many examples are known where this does not hold when $p=2$, but they are all for fusion and linking systems of groups of Lie type not of universal type, or for certain alternating groups (see the proof of [AOV1, Proposition 4.8]). In the appendix to BMO2, Oliver shows (in a case-bycase analysis) that whenever $G$ is a finite group of Lie type in odd characteristic, and of universal type, and $\mathcal{L}$ is the linking system for $G$ at $p=2$, then $\mu_{\mathcal{L}}$ is an isomorphism: a special case of the above question.

Question 7.10. Are there any exotic fusion systems which are fusion systems of blocks? See section 5.3, and also the discussions and partial results on this problem in [Ke1] and [KS].

Question 7.11. Does the gluing conjecture hold (Conjecture 5.22)? See the discussion in section 5.4 . 
Question 7.12. As described briefly in section 4.5, when $\mathcal{F}$ is a saturated fusion system over a finite $p$-group $S$, and $\mathcal{L}$ is a centric linking system associated to $\mathcal{F}$, the classifying space $B \mathcal{F}=|\mathcal{L}|$ of $\mathcal{F}$ is homotopy equivalent to a certain homotopy colimit over an orbit category $\mathcal{O}\left(\mathcal{F}^{c}\right)$. As one consequence, there is a spectral sequence

$$
E_{2}^{i j}=H^{i}\left(\mathcal{O}\left(\mathcal{F}^{c}\right) ; H^{j}\left(-; \mathbb{F}_{p}\right)\right) \quad \Longrightarrow \quad H^{i+j}\left(|\mathcal{L}| ; \mathbb{F}_{p}\right)
$$

Here, $H^{j}\left(-; \mathbb{F}_{p}\right)$ is the functor on $\mathcal{O}\left(\mathcal{F}^{c}\right)$ which sends a subgroup $P \leq S$ to $H^{j}\left(P ; \mathbb{F}_{p}\right) \cong H^{j}\left(B P ; \mathbb{F}_{p}\right)$. Also, by [BLO2, Theorem B], $H^{n}\left(|\mathcal{L}| ; \mathbb{F}_{p}\right) \cong$ $H^{0}\left(\mathcal{O}\left(\mathcal{F}^{c}\right) ; H^{n}\left(-; \mathbb{F}_{p}\right)\right.$ ) (i.e., the inverse limit of this functor). The sharpness conjecture for $H^{*}\left(|\mathcal{L}| ; \mathbb{F}_{p}\right)$ states that in the above spectral sequence, $E_{2}^{i j}=0$ for all $i>0$ and all $j \geq 0$. This is true when $\mathcal{F}$ is realizable by a theorem of Dwyer [Dw, Theorem 10.3], but it is still unknown whether or not it holds for arbitrary fusion systems.

\section{About the Authors}

Michael Aschbacher is the Shaler Arthur Hanisch Professor of Mathemematics, Emeritus, at California Institute of Technology (Caltech).

Bob Oliver is professor emeritus at Université Paris 13. His research interests are centered around algebraic topology and its connections to finite group theory.

\section{REFERENCES}

[Alp1] J. L. Alperin, Sylow intersections and fusion, J. Algebra 6 (1967), 222-241. MR0215913

[Alp2] J. L. Alperin, Local representation theory, Cambridge Studies in Advanced Mathematics, vol. 11, Cambridge University Press, Cambridge, 1986. Modular representations as an introduction to the local representation theory of finite groups. MR:860771

[Alp3] J. L. Alperin, Weights for finite groups, The Arcata Conference on Representations of Finite Groups (Arcata, Calif., 1986), Proc. Sympos. Pure Math., vol. 47, Amer. Math. Soc., Providence, RI, 1987, pp. 369-379. MR933373

[AB] J. L. Alperin and Michel Broué, Local methods in block theory, Ann. of Math. (2) 110 (1979), no. 1, 143-157, DOI 10.2307/1971248. MR541333

[ABC] Tuna Altınel, Alexandre V. Borovik, and Gregory Cherlin, Simple groups of finite Morley rank, Mathematical Surveys and Monographs, vol. 145, American Mathematical Society, Providence, RI, 2008. MR2400564

[AOV1] Kasper K. S. Andersen, Bob Oliver, and Joana Ventura, Reduced, tame and exotic fusion systems, Proc. Lond. Math. Soc. (3) 105 (2012), no. 1, 87-152, DOI 10.1112/plms/pdr065. MR2948790

[AOV2] K. Andersen, B. Oliver, and J. Ventura, Reduced fusion systems over 2-groups of small order (in preparation).

[A1] Michael Aschbacher, A characterization of Chevalley groups over fields of odd order, Ann. of Math. (2) 106 (1977), no. 2, 353-398. MR0498828

[A2] Michael Aschbacher, Finite group theory, Cambridge Studies in Advanced Mathematics, vol. 10, Cambridge University Press, Cambridge, 1986. MR895134

[A3] Michael Aschbacher, Normal subsystems of fusion systems, Proc. Lond. Math. Soc. (3) 97 (2008), no. 1, 239-271, DOI 10.1112/plms/pdm057. MR 2434097

[A4] Michael Aschbacher, The generalized Fitting subsystem of a fusion system, Mem. Amer. Math. Soc. 209 (2011), no. 986, vi+110, DOI 10.1090/S0065-9266-2010-006215. MR 2752788

[A5] Michael Aschbacher, Generation of fusion systems of characteristic 2-type, Invent. Math. 180 (2010), no. 2, 225-299, DOI 10.1007/s00222-009-0229-z. MR2609243

[A6] Michael Aschbacher, $S_{3}$-free 2-fusion systems, Proc. Edinb. Math. Soc. (2) 56 (2013), no. 1, 27-48, DOI 10.1017/S0013091512000235. MR.3021403 
[A7] Michael Aschbacher, Fusion systems of $\mathbf{F}_{2}$-type, J. Algebra 378 (2013), 217-262, DOI 10.1016/j.jalgebra.2012.12.018. MR3017023

[A8] Michael Aschbacher, Classifying finite simple groups and 2-fusion systems, ICCM Not. 3 (2015), no. 1, 35-42. MR3385504

[A9] Michael Aschbacher, N-groups and fusion systems, J. Algebra 449 (2016), 264-320, DOI 10.1016/j.jalgebra.2015.10.011. MR3448175

[AC] Michael Aschbacher and Andrew Chermak, A group-theoretic approach to a family of 2-local finite groups constructed by Levi and Oliver, Ann. of Math. (2) 171 (2010), no. 2, 881-978, DOI 10.4007/annals.2010.171.881. MR2630058

[AKO] Michael Aschbacher, Radha Kessar, and Bob Oliver, Fusion systems in algebra and topology, London Mathematical Society Lecture Note Series, vol. 391, Cambridge University Press, Cambridge, 2011. MR2848834

[ALSS] Michael Aschbacher, Richard Lyons, Stephen D. Smith, and Ronald Solomon, The classification of finite simple groups. Groups of characteristic 2 type, Mathematical Surveys and Monographs, vol. 172, American Mathematical Society, Providence, RI, 2011. MR2778190

[Be1] D. J. Benson, Representations and cohomology. I, 2nd ed., Cambridge Studies in Advanced Mathematics, vol. 30, Cambridge University Press, Cambridge, 1998. Basic representation theory of finite groups and associative algebras. MR 1644252

[Be2] David J. Benson, Cohomology of sporadic groups, finite loop spaces, and the Dickson invariants, Geometry and cohomology in group theory (Durham, 1994), London Math. Soc. Lecture Note Ser., vol. 252, Cambridge Univ. Press, Cambridge, 1998, pp. 10-23, DOI 10.1017/CBO9780511666131.003. MR1709949

[BGH] David J. Benson, Jesper Grodal, and Ellen Henke, Group cohomology and control of p-fusion, Invent. Math. 197 (2014), no. 3, 491-507, DOI 10.1007/s00222-013-0489-5. MR3251827

[BK] A. K. Bousfield and D. M. Kan, Homotopy limits, completions and localizations, Lecture Notes in Mathematics, Vol. 304, Springer-Verlag, Berlin-New York, 1972. MR0365573

[Br1] Richard Brauer, Some applications of the theory of blocks of characters of finite groups. IV, J. Algebra 17 (1971), 489-521. MR0281806

[Br2] Richard Brauer, On 2-blocks with dihedral defect groups, Symposia Mathematica, Vol. XIII (Convegno di Gruppi e loro Rappresentazioni, INDAM, Rome, 1972), Academic Press, London, 1974, pp. 367-393. MR.0354838

[BCGLO1] Carles Broto, Natàlia Castellana, Jesper Grodal, Ran Levi, and Bob Oliver, Subgroup families controlling p-local finite groups, Proc. London Math. Soc. (3) 91 (2005), no. 2, 325-354, DOI 10.1112/S0024611505015327. MR2167090

[BCGLO2] C. Broto, N. Castellana, J. Grodal, R. Levi, and B. Oliver, Extensions of p-local finite groups, Trans. Amer. Math. Soc. 359 (2007), no. 8, 3791-3858 (electronic), DOI 10.1090/S0002-9947-07-04225-0. MR2302515

[BL] Carles Broto and Ran Levi, On spaces of self-homotopy equivalences of p-completed classifying spaces of finite groups and homotopy group extensions, Topology 41 (2002), no. 2, 229-255, DOI 10.1016/S0040-9383(00)00031-8. MR/1876889

[BLO1] Carles Broto, Ran Levi, and Bob Oliver, Homotopy equivalences of p-completed classifying spaces of finite groups, Invent. Math. 151 (2003), no. 3, 611-664, DOI 10.1007/s00222-002-0264-5. MR1961340

[BLO2] Carles Broto, Ran Levi, and Bob Oliver, The homotopy theory of fusion systems, J. Amer. Math. Soc. 16 (2003), no. 4, 779-856, DOI 10.1090/S0894-0347-03-00434-X. MR:1992826

[BLO3] Carles Broto, Ran Levi, and Bob Oliver, Discrete models for the p-local homotopy theory of compact Lie groups and p-compact groups, Geom. Topol. 11 (2007), 315427, DOI 10.2140/gt.2007.11.315. MR.2302494

[BLO4] Carles Broto, Ran Levi, and Bob Oliver, A geometric construction of saturated fusion systems, An alpine anthology of homotopy theory, Contemp. Math., vol. 399, Amer. Math. Soc., Providence, RI, 2006, pp. 11-40, DOI 10.1090/conm/399/07510. MR2222502 
[BLO5] Carles Broto, Ran Levi, and Bob Oliver, An algebraic model for finite loop spaces, Algebr. Geom. Topol. 14 (2014), no. 5, 2915-2981, DOI 10.2140/agt.2014.14.2915. MR 3276851

[BMO1] Carles Broto, Jesper M. Møller, and Bob Oliver, Equivalences between fusion systems of finite groups of Lie type, J. Amer. Math. Soc. 25 (2012), no. 1, 1-20, DOI 10.1090/S0894-0347-2011-00713-3. MR2833477

[BMO2] Carles Broto, Jesper M. Møller, and Broto Oliver, Automorphisms of fusion systems of finite simple groups of Lie type, arXiv:1601.04566

[BP] Michel Broué and Lluís Puig, A Frobenius theorem for blocks, Invent. Math. 56 (1980), no. 2, 117-128, DOI 10.1007/BF01392547. MR558864

[Bu] W. Burnside, The Theory of Groups of Finite Order, Cambridge Univ. Press (1897).

[CE] Henri Cartan and Samuel Eilenberg, Homological algebra, Princeton University Press, Princeton, N. J., 1956. MR0077480

[Ch] Andrew Chermak, Fusion systems and localities, Acta Math. 211 (2013), no. 1, 47139, DOI 10.1007/s11511-013-0099-5. MR3118305

[ChOS] Andrew Chermak, Bob Oliver, and Sergey Shpectorov, The linking systems of the Solomon 2-local finite groups are simply connected, Proc. Lond. Math. Soc. (3) 97 (2008), no. 1, 209-238, DOI 10.1112/plms/pdn011. MR.2434096

[CP] Murray Clelland and Christopher Parker, Two families of exotic fusion systems, J. Algebra 323 (2010), no. 2, 287-304, DOI 10.1016/j.jalgebra.2009.07.023. MR 2564839

[Cr1] David A. Craven, Control of fusion and solubility in fusion systems, J. Algebra 323 (2010), no. 9, 2429-2448, DOI 10.1016/j.jalgebra.2010.02.025. MR2602388

[Cr2] David A. Craven, The theory of fusion systems. An algebraic approach, Cambridge Studies in Advanced Mathematics, vol. 131, Cambridge University Press, Cambridge, 2011. MR2808319

[Cr3] David A. Craven, Normal subsystems of fusion systems, J. Lond. Math. Soc. (2) 84 (2011), no. 1, 137-158, DOI 10.1112/jlms/jdr004. MR2819694

[CG] David A. Craven and Adam Glesser, Fusion systems on small p-groups, Trans. Amer. Math. Soc. 364 (2012), no. 11, 5945-5967, DOI 10.1090/S0002-9947-2012-05580-2. MR2946938

[CrOS] D. Craven, B. Oliver, and J. Semeraro, Simple fusion systems over p-groups with abelian subgroup of index $p$ : II (in preparation).

[Da1] E. C. Dade, Blocks with cyclic defect groups, Ann. of Math. (2) 84 (1966), 20-48. MR0200355

[DGMP1] Antonio Díaz, Adam Glesser, Nadia Mazza, and Sejong Park, Glauberman's and Thompson's theorems for fusion systems, Proc. Amer. Math. Soc. 137 (2009), no. 2, 495-503, DOI 10.1090/S0002-9939-08-09690-1. MR2448569

[DGMP2] Antonio Díaz, Adam Glesser, Nadia Mazza, and Sejong Park, Control of transfer and weak closure in fusion systems, J. Algebra 323 (2010), no. 2, 382-392, DOI 10.1016/j.jalgebra.2009.09.028. MR2564845

[DRV] Antonio Díaz, Albert Ruiz, and Antonio Viruel, All p-local finite groups of rank two for odd prime p, Trans. Amer. Math. Soc. 359 (2007), no. 4, 1725-1764 (electronic), DOI 10.1090/S0002-9947-06-04367-4. MR2272147

[Dw] W. G. Dwyer, Sharp homology decompositions for classifying spaces of finite groups, Group representations: cohomology, group actions and topology (Seattle, WA, 1996), Proc. Sympos. Pure Math., vol. 63, Amer. Math. Soc., Providence, RI, 1998, pp. 197220, DOI 10.1090/pspum/063/1603159. MR1603159

[DK] W. G. Dwyer and D. M. Kan, Centric maps and realization of diagrams in the homotopy category, Proc. Amer. Math. Soc. 114 (1992), no. 2, 575-584, DOI 10.2307/2159684. MR.1070515

[DW] W. G. Dwyer and C. W. Wilkerson, A new finite loop space at the prime two, J. Amer. Math. Soc. 6 (1993), no. 1, 37-64, DOI 10.2307/2152794. MR1161306

[FF] Ramón J. Flores and Richard M. Foote, Strongly closed subgroups of finite groups, Adv. Math. 222 (2009), no. 2, 453-484, DOI 10.1016/j.aim.2009.05.005. MR.2538017

[Fo] Richard Foote, A characterization of finite groups containing a strongly closed 2-subgroup, Comm. Algebra 25 (1997), no. 2, 593-606, DOI 10.1080/00927879708825876. MR1428800 
[Gl1] George Glauberman, Central elements in core-free groups, J. Algebra 4 (1966), 403420. MR0202822

[Gl2] George Glauberman, A characteristic subgroup of a p-stable group, Canad. J. Math. 20 (1968), 1101-1135. MR0230807

[GbL] G. Glauberman and J. Lynd, Control of weak closure and existence and uniqueness of centric linking systems, Invent. Math. (to appear).

[Gd1] David M. Goldschmidt, A conjugation family for finite groups, J. Algebra 16 (1970), 138-142. MR0260869

[Gd2] David M. Goldschmidt, 2-fusion in finite groups, Ann. of Math. (2) 99 (1974), 70-117. MR.0335627

[Gz] A. Gonzalez, Finite approximations of p-local compact groups, Geom. Topol. (to appear).

[GH] Daniel Gorenstein and Koichiro Harada, Finite groups whose 2-subgroups are generated by at most 4 elements, American Mathematical Society, Providence, R.I., 1974. Memoirs of the American Mathematical Society, No. 147. MR0367048

[GLS1] Daniel Gorenstein, Richard Lyons, and Ronald Solomon, The classification of the finite simple groups, Mathematical Surveys and Monographs, vol. 40, American Mathematical Society, Providence, RI, 1994. MR 1303592

[GLS3] Daniel Gorenstein, Richard Lyons, and Ronald Solomon, The classification of the finite simple groups, Mathematical Surveys and Monographs, vol. 40, American Mathematical Society, Providence, RI, 1994. MR 1303592

[Gr] J. A. Green, On the indecomposable representations of a finite group, Math. Z. 70 (1958/59), 430-445. MR0131454

[He1] Ellen Henke, Recognizing $\mathrm{SL}_{2}(q)$ in fusion systems, J. Group Theory 13 (2010), no. 5, 679-702, DOI 10.1515/JGT.2010.017. MR2720198

[He2] Ellen Henke, Minimal fusion systems with a unique maximal parabolic, J. Algebra 333 (2011), 318-367, DOI 10.1016/j.jalgebra.2010.11.006. MR2785951

[He3] Ellen Henke, Products in fusion systems, J. Algebra 376 (2013), 300-319, DOI 10.1016/j.jalgebra.2012.11.037. MR3003728

[He4] Ellen Henke, A characterization of saturated fusion systems over abelian 2-groups, Adv. Math. 257 (2014), 1-5, DOI 10.1016/j.aim.2014.02.020. MR.3187642

[He5] E. Henke, Subcentric linking systems, arXiv:1506.01458

[JLL] Fabien Junod, Ran Levi, and Assaf Libman, Unstable Adams operations on p-local compact groups, Algebr. Geom. Topol. 12 (2012), no. 1, 49-74, DOI 10.2140/agt.2012.12.49. MR2889545

[Ke1] Radha Kessar, The Solomon system $\mathcal{F}_{\text {Sol }}(3)$ does not occur as fusion system of a 2-block, J. Algebra 296 (2006), no. 2, 409-425, DOI 10.1016/j.jalgebra.2005.09.026. MR2201049

[Ke2] Radha Kessar, Introducton to block theory, Group representation theory, EPFL Press, Lausanne, 2007, pp. 47-77. MR2336637

[KL] Radha Kessar and Markus Linckelmann, ZJ-theorems for fusion systems, Trans. Amer. Math. Soc. 360 (2008), no. 6, 3093-3106, DOI 10.1090/S0002-9947-08-04275-X. MR2379788

[KS] Radha Kessar and Radu Stancu, A reduction theorem for fusion systems of blocks, J. Algebra 319 (2008), no. 2, 806-823, DOI 10.1016/j.jalgebra.2006.05.039. MR2381811

[KP] Burkhard Külshammer and Lluís Puig, Extensions of nilpotent blocks, Invent. Math. 102 (1990), no. 1, 17-71, DOI 10.1007/BF01233419. MR1069239

[LL] Ran Levi and Assaf Libman, Existence and uniqueness of classifying spaces for fusion systems over discrete p-toral groups, J. Lond. Math. Soc. (2) 91 (2015), no. 1, 47-70, DOI 10.1112/jlms/jdu062. MR 3338608

[LO] R. Levi and B. Oliver, Construction of 2-local finite groups of a type studied by Solomon and Benson, Geometry and Topology 6 (2002), 917-990 MR.1943386 and Correction to: "Construction of 2-local finite groups of a type studied by Solomon and Benson", Geometry and Topology 9 (2005), 2395-2415 MR2209376

[LR] Ran Levi and Kári Ragnarsson, p-local finite group cohomology, Homology Homotopy Appl. 13 (2011), no. 1, 223-257, DOI 10.4310/HHA.2011.v13.n1.a8. MR2803873

[Lb1] Assaf Libman, The gluing problem does not follow from homological properties of $\Delta_{p}(G)$, Homology, Homotopy Appl. 12 (2010), no. 1, 1-10. MR 2594678 
[Lb2] Assaf Libman, The gluing problem in the fusion systems of the symmetric, alternating and linear groups, J. Algebra $\mathbf{3 4 1}$ (2011), 209-245, DOI 10.1016/j.jalgebra.2011.06.007. MR2824518

[Li1] Markus Linckelmann, Fusion category algebras, J. Algebra 277 (2004), no. 1, 222-235, DOI 10.1016/j.jalgebra.2003.12.010. MR.2059628

[Li2] Markus Linckelmann, On $H^{*}\left(\mathcal{C} ; k^{\times}\right)$for fusion systems, Homology, Homotopy Appl. 11 (2009), no. 1, 203-218. MR2506133

[Lyn1] Justin Lynd, The Thompson-Lyons transfer lemma for fusion systems, Bull. Lond. Math. Soc. 46 (2014), no. 6, 1276-1282, DOI 10.1112/blms/bdu083. MR.3291264

[Lyn2] Justin Lynd, A characterization of the 2-fusion system of $L_{4}(q)$, J. Algebra 428 (2015), 315-356, DOI 10.1016/j.jalgebra.2014.10.046. MR.3314296

[McL] Saunders Mac Lane, Homology, Classics in Mathematics, Springer-Verlag, Berlin, 1995. Reprint of the 1975 edition. MR1344215

[MP] John Martino and Stewart Priddy, Unstable homotopy classification of $B G_{p}$, Math. Proc. Cambridge Philos. Soc. 119 (1996), no. 1, 119-137, DOI 10.1017/S030500410007403X. MR1356164

[O1] Bob Oliver, Equivalences of classifying spaces completed at odd primes, Math. Proc. Cambridge Philos. Soc. 137 (2004), no. 2, 321-347, DOI 10.1017/S0305004104007728. MR2092063

[O2] Bob Oliver, Equivalences of classifying spaces completed at the prime two, Mem. Amer. Math. Soc. 180 (2006), no. 848, vi+102, DOI 10.1090/memo/0848. MR2203209

[O3] Bob Oliver, Extensions of linking systems and fusion systems, Trans. Amer. Math. Soc. 362 (2010), no. 10, 5483-5500, DOI 10.1090/S0002-9947-2010-05022-6. MR2657688

[O4] Bob Oliver, Existence and uniqueness of linking systems: Chermak's proof via obstruction theory, Acta Math. 211 (2013), no. 1, 141-175, DOI 10.1007/s11511-013-0100-3. MR3118306

[O5] Bob Oliver, Simple fusion systems over p-groups with abelian subgroup of index $p$ : I, J. Algebra 398 (2014), 527-541, DOI 10.1016/j.jalgebra.2013.09.012. MR 3123784

[O6] Bob Oliver, Reduced fusion systems over 2-groups of sectional rank at most 4, Mem. Amer. Math. Soc. 239 (2016), no. 1131, v+100, DOI 10.1090/memo/1131. MR3431946

[O7] Bob Oliver, Reductions to simple fusion systems, arXiv: 1601.07978

[O8] Bob Oliver, Automorphisms of fusion systems of sporadic simple groups, arXiv: 1604.05681

[OV1] Bob Oliver and Joana Ventura, Extensions of linking systems with p-group kernel, Math. Ann. 338 (2007), no. 4, 983-1043, DOI 10.1007/s00208-007-0104-4. MR2317758

[OV2] Bob Oliver and Joana Ventura, Saturated fusion systems over 2-groups, Trans. Amer. Math. Soc. 361 (2009), no. 12, 6661-6728, DOI 10.1090/S0002-9947-09-04881-8. $\operatorname{MR} 2538610$

[Ols] Jørn Børling Olsson, On 2-blocks with quaternion and quasidihedral defect groups, J. Algebra 36 (1975), no. 2, 212-241. MR0376841

[On] Silvia Onofrei, Saturated fusion systems with parabolic families, J. Algebra 348 (2011), 61-84, DOI 10.1016/j.jalgebra.2011.09.028. MR2852232

[Pa] Sejong Park, The gluing problem for some block fusion systems, J. Algebra 323 (2010), no. 6, 1690-1697, DOI 10.1016/j.jalgebra.2010.01.003. MR2588132

[PS] Chris Parker and Gernot Stroth, A family of fusion systems related to the groups $\mathrm{Sp}_{4}\left(p^{a}\right)$ and $\mathrm{G}_{2}\left(p^{a}\right)$, Arch. Math. (Basel) 104 (2015), no. 4, 311-323, DOI 10.1007/s00013-015-0751-8. MR3325764

[Pg1] Lluís Puig, Structure locale dans les groupes finis (French), Bull. Soc. Math. France Suppl. Mém. 47 (1976), 132. MR0450410

[Pg2] Lluís Puig, Nilpotent blocks and their source algebras, Invent. Math. 93 (1988), no. 1, 77-116, DOI 10.1007/BF01393688. MR943924

[Pg3] Lluis Puig, The hyperfocal subalgebra of a block, Invent. Math. 141 (2000), no. 2, 365-397, DOI 10.1007/s002220000072. MR.1775217

[Pg4] Lluis Puig, Frobenius categories, J. Algebra 303 (2006), no. 1, 309-357, DOI 10.1016/j.jalgebra.2006.01.023. MR2253665 
[Pg5] Lluís Puig, Frobenius categories versus Brauer blocks, The Grothendieck group of the Frobenius category of a Brauer block, Progress in Mathematics, vol. 274, Birkhäuser Verlag, Basel, 2009. MR2502803

[Q] Daniel Quillen, The spectrum of an equivariant cohomology ring. I, II, Ann. of Math. (2) 94 (1971), 549-572; ibid. (2) 94 (1971), 573-602. MR0298694

[Rg] Kári Ragnarsson, Classifying spectra of saturated fusion systems, Algebr. Geom. Topol. 6 (2006), 195-252, DOI 10.2140/agt.2006.6.195. MR2199459

[RSt] Kári Ragnarsson and Radu Stancu, Saturated fusion systems as idempotents in the double Burnside ring, Geom. Topol. 17 (2013), no. 2, 839-904, DOI 10.2140/gt.2013.17.839. MR3070516

[RS] K. Roberts and S. Shpectorov, On the definition of saturated fusion systems, J. Group Theory 12 (2009), no. 5, 679-687, DOI 10.1515/JGT.2009.003. MR.2554760

[Rz] Albert Ruiz, Exotic normal fusion subsystems of general linear groups, J. Lond. Math. Soc. (2) 76 (2007), no. 1, 181-196, DOI 10.1112/jlms/jdm051. MR2351616

[RV] Albert Ruiz and Antonio Viruel, The classification of p-local finite groups over the extraspecial group of order $p^{3}$ and exponent p, Math. Z. 248 (2004), no. 1, 45-65, DOI 10.1007/s00209-004-0652-1. MR2092721

[Sa] Benjamin Sambale, Fusion systems on metacyclic 2-groups, Osaka J. Math. 49 (2012), no. 2, 325-329. MR2945751

[Sg] Graeme Segal, Classifying spaces and spectral sequences, Inst. Hautes Études Sci. Publ. Math. 34 (1968), 105-112. MR0232393

[So] Ronald Solomon, Finite groups with Sylow 2-subgroups of type 3, J. Algebra 28 (1974), 182-198. MR0344338

[St] Radu Stancu, Control of fusion in fusion systems, J. Algebra Appl. 5 (2006), no. 6, 817-837, DOI 10.1142/S0219498806002034. MR2286725

[SSy] Radu Stancu and Peter Symonds, Fusion systems for profinite groups, J. Lond. Math. Soc. (2) 89 (2014), no. 2, 461-481, DOI 10.1112/jlms/jdt074. MR3188628

[Su] Dennis P. Sullivan, Geometric topology: localization, periodicity and Galois symmetry, K-Monographs in Mathematics. The 1970 MIT notes, vol. 8, Springer, Dordrecht, 2005. Edited and with a preface by Andrew Ranicki. MR2162361

[T] John G. Thompson, Nonsolvable finite groups all of whose local subgroups are solvable, Bull. Amer. Math. Soc. 74 (1968), 383-437. MR0230809

[Xu] Xingzhong Xu, Saturated fusion systems over a class of finite p-groups, J. Algebra 413 (2014), 135-152, DOI 10.1016/j.jalgebra.2014.05.003. MR3216603

Department of Mathematics, California Institute of Technology, Pasadena, CaliFORNIA 91125

E-mail address: asch@caltech.edu

Université Paris 13, Sorbonne Paris Cité, LAGA, UMr 7539 du CNRS, 99, Av. J.-B. Clément, 93430 Villetaneuse, France

E-mail address: bobol@math.univ-paris13.fr 